\title{
A Fluid-Structure Interaction Analysis of the Human Aortic Arch Under InERTial LOAD
}

by

\author{
Jonathan M. Zalger \\ Bachelor of Engineering, Ryerson University, 2008 \\ A thesis \\ presented to Ryerson University \\ in partial fulfillment of the \\ requirements for the degree of \\ Master of Applied Science \\ in the Program of \\ Aerospace Engineering
}

Toronto, Ontario, Canada, 2010

C.Jonathan M. Zalger 2010 

I hereby declare that I am the sole author of this thesis.

I authorize Ryerson University to lend this thesis to other institutions or individuals for the purpose of scholarly research.

I further authorize Ryerson University to reproduce this thesis by photocopying or by other means, in total or in part, at the request of other institutions or individuals for the purpose of scholarly research. 

A Fluid-Structure Interaction Analysis of the Human Aortic Arch Under Inertial Load

Master of Applied Science 2010

Jonathan M. Zalger

Aerospace Engineering

Ryerson University

\begin{abstract}
Presented is an investigation into the use of numerical methods for modelling the effects of inertial load on the human cardiovascular system. An anatomically correct geometry was developed based on threedimensional computed tomography (CT) data and independent meshes were created for the solid and fluid regimes. These domains were simulated using independent solvers and subsequently coupled using an intermediate data transfer algorithm. At the inlet of the arch, a pulsatile velocity boundary condition was enforced replicating the cardiac cycle. Time invariant, resistive boundary conditions were used at all outlets and a linear isotropic constitutive model was used for tissue response. Verification was conducted by comparing simulation results at standard earth gravity $\left(9.81 \mathrm{~m} / \mathrm{s}^{2}\right)$ with published values for velocity, mass flow rate, deformation, and qualitative flow behaviour. The presented Huid-structure interaction (FSI) model shows strong agreement with accepted normal values. Inertial load was then applied along the longitudinal axis of the arch in multiples of standard gravity to a maximum of $8+\mathrm{Gz}$. This load increased arch flow velocities, and reduced mass flow in the ascending brachiocephalic and carotid arteries. Blood flow from the arch to the upper body and brain ceased near $8+\mathrm{Gz}$. Although the presented results are preliminary, the feasibility of such an analysis has been successfully demonstrated.
\end{abstract}





\section{Acknowledgements}

I would first like to extend tremendous gratitude to my advisor, Dr. Jason Lassaline for agreeing to venture into this new field of research. His immense technical expertise and commitment to graduate students is unrivaled. I will always appreciate your open door and support over the past few years which has made this thesis possible.

I would also like to extend my sincere thanks to my thesis review committee, Dr. Paul Walsh, Dr. Seyed M. Hashemi, and the program director, Dr. Jeffrey Yokota. Thank you again for your time in reviewing this thesis.

In addition, I would like to acknowledge the Natural Sciences and Research Council of Canada (NSERC) for partial funding of this research. This project was also made possible by the facilities of the Shared Hierarchical Academic Research Computing Network (SHARCNET, www.sharcnet.ca) and Compute/Calcul Canada. 



\section{Dedication}

This thesis is dedicated to my family and friends for their endless support and patience throughout the completion of this degree. 



\section{Contents}

\begin{tabular}{l|l}
1 Introduction & 1
\end{tabular}

2 Background

2.1 Basic Anatomy and Physiology . . . . . . . . . . . . . . . . . . . . . . . . 3

2.2 Human Response to Inertial Load . . . . . . . . . . . . . . . . . . . . . . . . . . . . . 5

2.3 Fundamental BioHuid Dynamics . . . . . . . . . . . . . . . . . . . . . . . . . . . . . 7

2.4 Biosolid Mechanics . . . . . . . . . . . . . . . . . . . . . . . . . . . . . . . 10

2.4.1 Arterial Structure . . . . . . . . . . . . . . . . . . . . . . 10

2.4 .2 Elastin . . . . . . . . . . . . . . . . . . . . . . . . 10

2.4 .3 Collagen . . . . . . . . . . . . . . . . . . . . . . . 11

2.5 Numerical Modelling . . . . . . . . . . . . . . . . . . . . . . . . . . . . . . . . . . . . . . 12

2.6 Analysis of Inertial Loading . . . . . . . . . . . . . . . . . . . . . . . . . 13

3 Geometry and Mesh Generation 17

3.1 Geometry . . . . . . . . . . . . . . . . . . . . . . . . . . . . . 17

3.1.1 Computed Tomography (CT) Based Modelling . . . . . . . . . . . . . . . . . . . . 17

3.1.2 Magnetic Resonance Imaging (MRI) . . . . . . . . . . . . . . . . . . . . . . . . 18

3.1.3 Segmentation and Model Development . . . . . . . . . . . . . . . . . . . . . . . . . 19

B.1.4 Imaging Considerations . . . . . . . . . . . . . . . . . . . . . . . . . . 19

3.2 Meshing . . . . . . . . . . . . . . . . . . . . . . . . . . 21

B.2.1 Solid Meshing . . . . . . . . . . . . . . . . . . . . . . . . . . . . . . . . . . . 22

3.2.2 Fluid Meshing . . . . . . . . . . . . . . . . . . . . . . . . 23

4 Methodology 25

4.1 CED Solution . . . . . . . . . . . . . . . . . . . . . . . . . . . . . . . . . 25

4.1.1 Inlet Boundary Conditions . . . . . . . . . . . . . . . . . . . . . . . . . . 25

4.1.2 Outlet Boundary Conditions . . . . . . . . . . . . . . . . . . . . . . . 26

4.1.3 Solution Parameters . . . . . . . . . . . . . . . . . . . . . . . . . . 30

4.2 Finite Element Solution . . . . . . . . . . . . . . . . . . . . . . . . . . . . . . . . 30

4.2 .1 Linear Constitutive Models . . . . . . . . . . . . . . . . . . . . . . . . . 30 
4.2 .2 Hyperelastic Representations . . . . . . . . . . . . . . . . . . . 31

4.2 .3 Nonlinear Anisotropic Models . . . . . . . . . . . . . . . . . . . . . . . 33

4.2 .4 FEM Solution Methodology . . . . . . . . . . . . . . . . . . . . . . . . . . 33

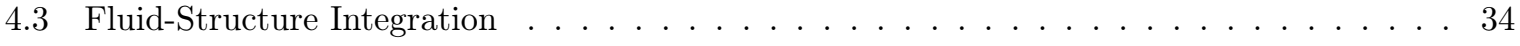

4.3 .1 Boundary Data Exchange $\ldots \ldots \ldots \ldots \ldots$. . . . . . . . . . . . . . . . . . . . . .

$\begin{array}{lll}5 & \text { Results } & \mathbf{3 7}\end{array}$

5.1 Mesh Independence Study . . . . . . . . . . . . . . . . . . . . . . . . 37

5.2 Steady State Results . . . . . . . . . . . . . . . . . . . . . . . . . . . 37

5.2 .1 Steady CFD . . . . . . . . . . . . . . . . . . . . . . . 37

522 Static FEM Results . . . . . . . . . . . . . . . . . . . . . 39

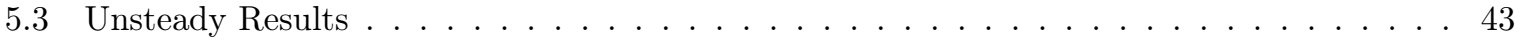

5.1 Overview . . . . . . . . . . . . . . . . . . . . 43

5.3 .2 Velocity and Mass Flow $\ldots \ldots \ldots \ldots \ldots \ldots \ldots \ldots$

5.3 .3 Pressure . . . . . . . . . . . . . . . . . . . . . . . 45

5.3 .4 Turbulence and Flow Behaviour . . . . . . . . . . . . . . . . . 45

5.4 Comparison of Unsteady vs. Steady Results . . . . . . . . . . . . . . . . 50

5.5 Coupled Fluid-Structure Results $\ldots \ldots \ldots \ldots \ldots \ldots \ldots \ldots \ldots$

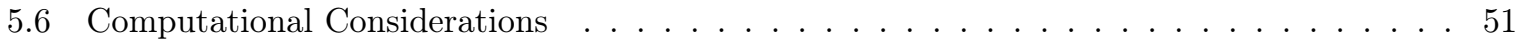

6 Conclusions $\quad 53$

6.1 Improving Solution Etficiency $\ldots \ldots \ldots \ldots \ldots \ldots \ldots \ldots$

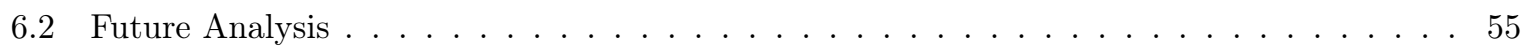

$\begin{array}{ll}\text { References } & 69\end{array}$

$\begin{array}{ll}\text { Glossary } & 71\end{array}$

\begin{tabular}{ll}
\hline Acronyms & 73
\end{tabular}

$\begin{array}{ll}\text { Index } & 75\end{array}$ 


\section{List of Tables}

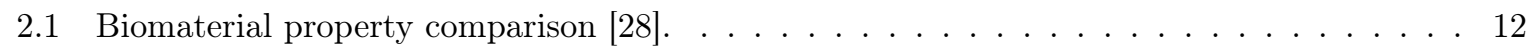

4.1 Resistive boundary coefticients. . . . . . . . . . . . . . . . . . . . . . . . 27

4.2 Steady and unsteady CFD simulation parameters $\ldots \ldots \ldots \ldots \ldots$

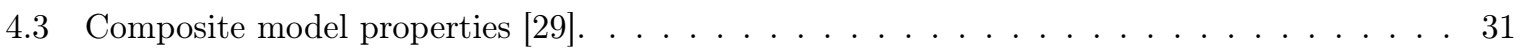

4.4 Linear isotropic material parameters. . . . . . . . . . . . . . . . . . . . . . 34

5.1 Summary of static linear elastic results $\ldots \ldots \ldots \ldots \ldots \ldots \ldots$

5.2 Summary of static composite model results. . . . . . . . . . . . . . . . . . . . 41

5.3 Comparison of peak aortic arch velocities by various modalities. . . . . . . . . . . . . 44

5.4 CFD solution scalability test conducted on the SHARCNET Saw supercomputer. . . . . . 52 



\section{List of Figures}

2.1 A cut-away view of the human heart and valves $90 \ldots \ldots \ldots \ldots \ldots$

2.2 The three components of blood $87 \ldots \ldots \ldots \ldots \ldots \ldots$

2.3 Gravitational force axis $22 . \ldots \ldots \ldots \ldots \ldots \ldots \ldots$

2.4 Effects of positive $\mathrm{G}$ acceleration in the longitudinal $\mathrm{Z}$ direction $4 . \ldots \ldots \ldots$

2.5 Physical structure of a normal human artery $|37| \ldots \ldots \ldots \ldots \ldots \ldots \ldots \ldots$

\begin{tabular}{|lll|l|l|}
\hline 2.6 & Diagram of the systemic arterial tree model developed by Snyder and Rideout, 1968 & 777
\end{tabular}

$3.1 \quad$ An illustration of image acquisition using MRI $77 \ldots \ldots \ldots \ldots \ldots$

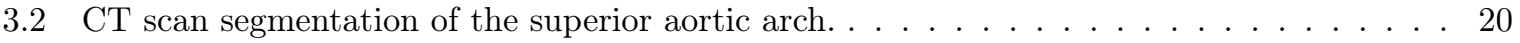

3.3 Rough surface reconstruction prior to smoothing operations. . . . . . . . . . . . . . . 20

3.4 Completed NURBS based model. . . . . . . . . . . . . . . . . . . . 21

3.5 Localized mesh sizing errors due to NURBS patch topology. . . . . . . . . . . . . . . . 22

3.6 Overview of the quadrilateral solid mesh. . . . . . . . . . . . . . . . . . . 23

3.7 Closeup of inflated boundary layer near the left subclavian artery boundary. . . . . . . . . 24

3.8 Section view of tinal thuid mesh . . . . . . . . . . . . . . . . . . . 24

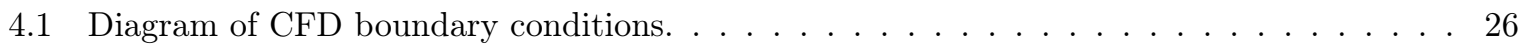

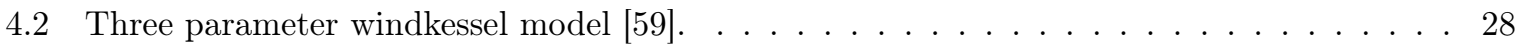

4.3 Olufsen structured tree model 59 . . . . . . . . . . . . . . . . . . . . . . . . . . . . . 29

$4.4 \quad$ Staggered weak coupling algorithm used by MpCCI 6 . . . . . . . . . . . . . . . . 35

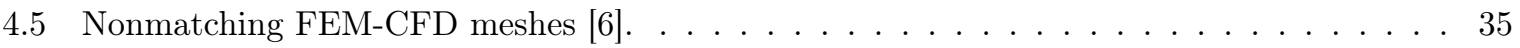

5.1 CFD mesh independence study showing LCC mass flow rate verses mesh density. . . . . . 38

5.2 Steady mass flow distribution across boundaries. . . . . . . . . . . . . . . . . . . 39

5.3 Steady velocity streamlines. . . . . . . . . . . . . . . . . . . . . . . . 40

5.4 Contours of static pressure (steady state) . . . . . . . . . . . . . . . . . . . . . 40

5.5 Comparison of wall displacement between homogeneous and composite linear elastic models. 42

5.6 Comparison of von Mises stress between linear and composite elastic models. . . . . . . . 42

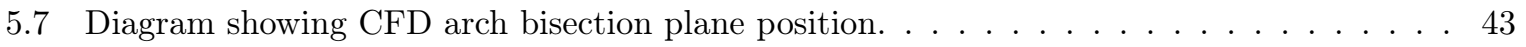

5.8 Velocity vectors across the mid-plane of the arch. . . . . . . . . . . . . . . . 44

5.9 Effect of inertial load on average velocity at the top section of the arch. . . . . . . . . 45 
5.10 Effect of inertial load on unsteady mass flow through the left common carotid artery. . . . 46

5.11 Reduction in mass flow rate through the left common carotid artery with increasing inertial

load compared with a 1 G baseline. . . . . . . . . . . . . . . . . . . . . 46

5.12 Pressure contours at systole under standard gravity conditions. . . . . . . . . . . . . 47

5.13 Variation in arch pressure under gravitational load. . . . . . . . . . . . . . . . . 47

5.14 Systolic $y+$ contours during an unsteady standard gravity simulation. . . . . . . . . . 48

5.15 Comparison of velocity streamlines in the aortic arch for $1 \mathrm{G}_{\mathrm{z}}$ and $6 \mathrm{G}_{\mathrm{z}} \ldots \ldots \ldots$. . . . 49

5.16 Comparison of systolic displacement under inertial load. . . . . . . . . . . . . . . 51

5.17 Comparison of systolic von Mises stress under inertial load. . . . . . . . . . . . . . 52

5.18 Scalability of the CFD solution in a parallel environment. . . . . . . . . . . . . . 52

B.1 Diagram of the cardiovascular system $5 \mid . \ldots \ldots \ldots \ldots \ldots \ldots$

B.2 Illustration of the heart and aortic arch $32 \mid \ldots \ldots \ldots \ldots \ldots$ 


\section{List of Appendices}

\begin{tabular}{|ll|}
\hline A Unsteady Velocity Relation & 57
\end{tabular}

$\begin{array}{ll}\text { B Additional Anatomic References } & 59\end{array}$ 



\title{
List of Symbols
}

\author{
Alphanumeric Symbols \\ A area, $\mathrm{m}^{2}$ \\ a curve fit coefficient \\ $a_{n} \quad$ Fourier curve fit parameter \\ $b \quad$ curve fit coefficient \\ $b_{n} \quad$ Fourier curve fit parameter \\ $C_{a} \quad$ peripheral vascular resistance, $\mathrm{kg} / \mathrm{m}^{4} \mathrm{~s}$ \\ d diameter, $\mathrm{m}$ \\ d material compressibility parameter \\ E Young's modulus, $\mathrm{MPa}$ \\ $F_{i j} \quad$ volumetric component of material deformation gradient \\ G gravitational force, $\mathrm{N}$ \\ G shear modulus, $\mathrm{Pa}$ \\ $g \quad$ standard earth gravity, $\mathrm{m} / \mathrm{s}^{2}$ \\ $G_{x}$ gravitational force in the $\mathrm{X}$ axis (front-to-back), N \\ $G_{y}$ gravitational force in the $\mathrm{Y}$ axis (side-to-side), $\mathrm{N}$ \\ $G_{z} \quad$ gravitational force in the $\mathrm{Z}$ axis (head-to-foot), N
}




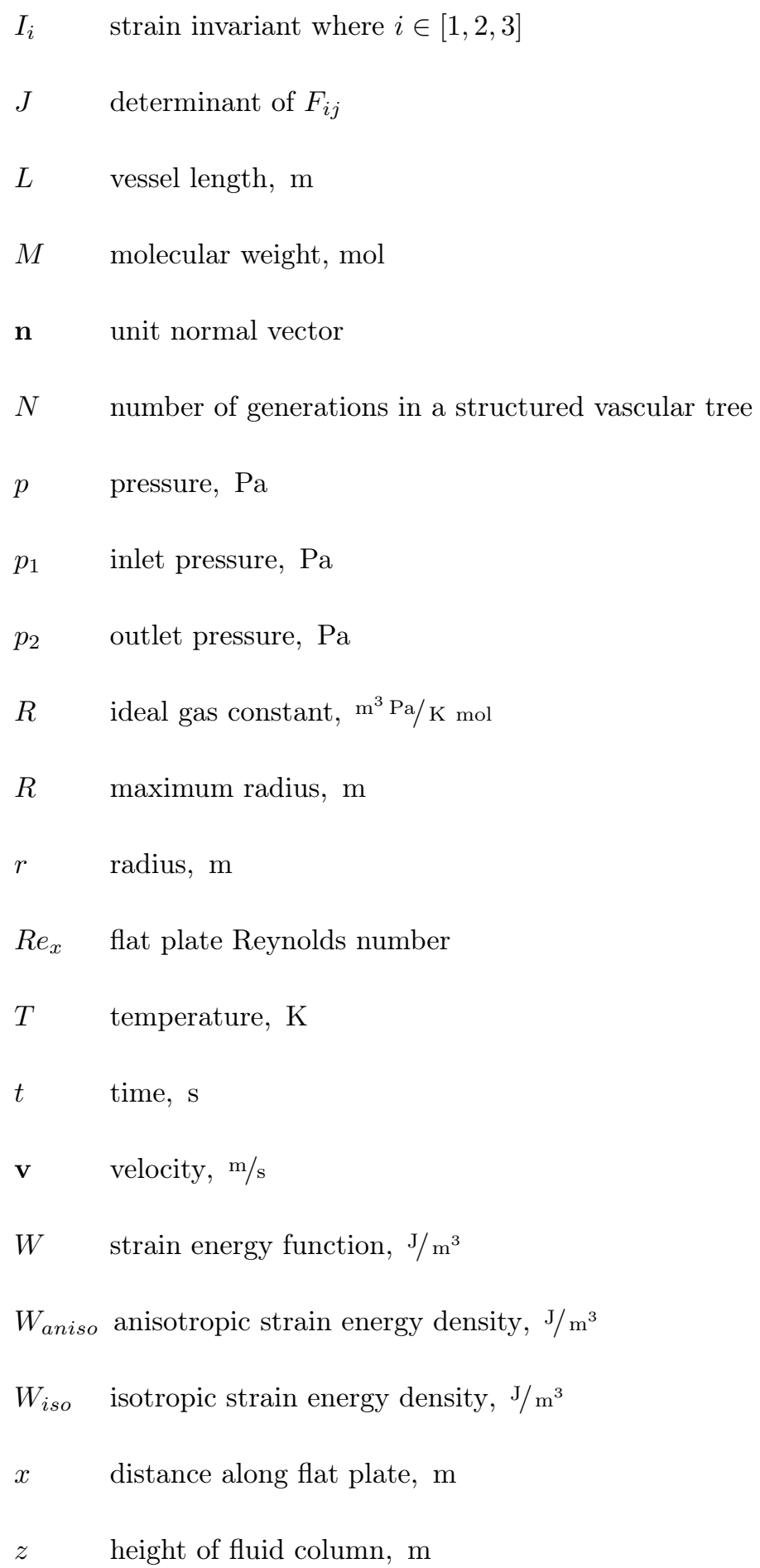

\section{Greek Symbols}

$\alpha \quad$ Wormersley number 


$\begin{array}{ll}\delta & \text { boundary layer thickness, m } \\ \delta \Omega & \text { domain boundary } \\ \epsilon_{i j} & \text { strain tensor } \\ \kappa & \text { bulk modulus, } \mathrm{Pa} \\ \mu & \text { fluid viscosity, } \mathrm{kg} / \mathrm{ms} \\ \mu & \text { shear modulus, } \mathrm{Pa} \\ \nu & \text { Poisson ratio } \\ \omega & \text { heart rate, } 1 / \mathrm{s} \\ \rho & \text { fluid density, } \mathrm{kg} / \mathrm{m}^{3} \\ \sigma_{i j} & \text { stress tensor, } \mathrm{Pa}\end{array}$





\section{Chapter 1}

\section{Introduction}

Examining the long term physiologic implications of aerospace environments on the cardiovascular system requires accurate modelling and analysis. In this study, the interaction of tissue response and fluid dynamics in the aortic arch are investigated under the influence of inertial load. The ability to accurately simulate such scenarios would enable exciting new pathways for research in aerospace medicine.

Predicting the safe level of gravitational $(G)$ force exposure has been a paramount concern for many years. It is well known that high levels of exposure can precipitate symptoms in the body such as narrowing or loss of vision, unconsciousness, and eventually death [22]. Determining an individual's tolerance to $\mathrm{G}$ has historically been measured experimentally through the use of human centrifuges where subjects can be exposed to increasing inertial force in a controlled setting under close medical supervision. However, in a research setting, such apparatus brings high costs and inherent limitations. Many centrifuges are designed for a limited load axis, and are restricted in the rates and limits of load application. Furthermore, the ability for instrumentation of a subject may be limited by size, weight, or in some cases ethical concerns. These limitations may hinder accurate tolerance assessment in future, more complex flight profiles. The use of numerical analysis would not only allow greater freedom of test scenarios, but the ability for integration with other design tools, such as a flight dynamics model. In such a case, optimization strategies could even be employed to develop the safest, most efficient flight envelope.

Current experimental analysis is also somewhat limited in its ability to elucidate low level information regarding flight physiology. Information such as flow behaviour and tissue response is very difficult to measure experimentally under load, yet could reveal substantial clues as to the long term effects of exposure to G. For example, numerous medical studies (under normal gravity) have shown a connection between wall shear stress in an artery and the development of long-term cardiovascular disease [65] [90] [67][[79][67]. Currently no public research has been published investigating effects of inertial load on such a connection. Studying the interaction of inertial load on properties such as wall shear 
stress may provide medical researchers a greater understanding of cardiovascular health in pilots.

To facilitate such research, a numerical model is presented with the goal of incorporating a computational fluid dynamics (CFD) analysis of blood flow coupled with a finite element (FE) analysis of the surrounding tissue wall. By simulating the fluid-structure interaction, the intent is to create a realistic model in which varying levels of inertial load can be applied and physiological parameters simulated. To achieve the highest fidelity possible, model geometry will be derived from computed tomography (CT) scans of a healthy male. As the intent of the study is not to be patient specific, the geometry is assumed to be representative in size and shape of a normal healthy aorta from which generalized conclusions can be formed.

This geometry was subdivided into two distinct grids for the solid and fluid domain. Each grid is meshed with the ideal characteristics specific to the domain. This allows special regions such as the fluid boundary layer, or areas of stress concentrations to be accurately meshed.

Each domain was solved in a coupled but segregated manor with a distinct solver for each regime. This allows the choice of solver to be made based on its strengths in dealing with the physics of the problem. For tissue modelling, MSC. Marc by MSC Software Corp. was chosen for its relative strength in nonlinear solutions. FLUENT by ANSYS Inc. was chosen to simulate the hemodynamics for its strong extensibility in terms of boundary condition and turbulence modelling. Both solvers have strong industry reputations and have been heavily evaluated. MpCCI by Fraunhofer SCAI was chosen as the intermediate "code coupling" solution responsible for transferring forces and displacements between each domain. Within this document, solutions will be presented in stages of increasing fidelity beginning with decoupled, static, and steady solutions, progressing towards a fully coupled unsteady solution.

A simulation conducted at standard earth gravity was used for validation purposes. Parameters including pressure, mass flow rate, velocity, and wall shear stress were used to evaluate the efficacy of the model in addition to qualitative comparison to experimental data, as presented in Section 15 . Based on the collected results, conclusions were drawn and the implications discussed along with avenues for future research. 


\section{Chapter 2}

\section{Background}

\subsection{Basic Anatomy and Physiology}

\section{Overview}

The human circulatory system is analogous to many other hydraulic systems seen in engineering. It can be generalized to three main components: a pump, a fluid, and a piping structure (Fig. B.J). In the body, these components are the heart, blood, and vascular system, respectively. The system is responsible for transporting nutrients, waste, oxygen, and carbon dioxide, as well as acting in thermoregulation and immune activities. The unique organic nature of the system creates several interesting, yet complex challenges when being analyzed in an engineering context [41]].

\section{The Heart}

The heart is the muscle which drives the circulatory system in the body. It is comprised of four chambers, two for receiving blood, two for pumping (Fig. ए.]). The left and right atria intake blood from the lungs and body respectively, while the right and left ventricles contract to force blood to the lungs and the body (Fig. B.2).

The contraction of the heart is triggered electrically due to an action potential developed in cardiac cells from a property known as automaticity. The charge propagates across the heart irritating the muscles in the chambers causing them to contract beginning with the atria followed by the ventricles. The heart beats in a periodic cycle at a normal rate between 60-100 beats per minute. This beat creates pulsatile flow in much of the body's vasculature. During a beat, known as systole, pressures in the ascending aorta, the vessel exiting the heart, normally average around $120 \mathrm{mmHg}$ or $16 \mathrm{kPa}$ gauge [28]. 


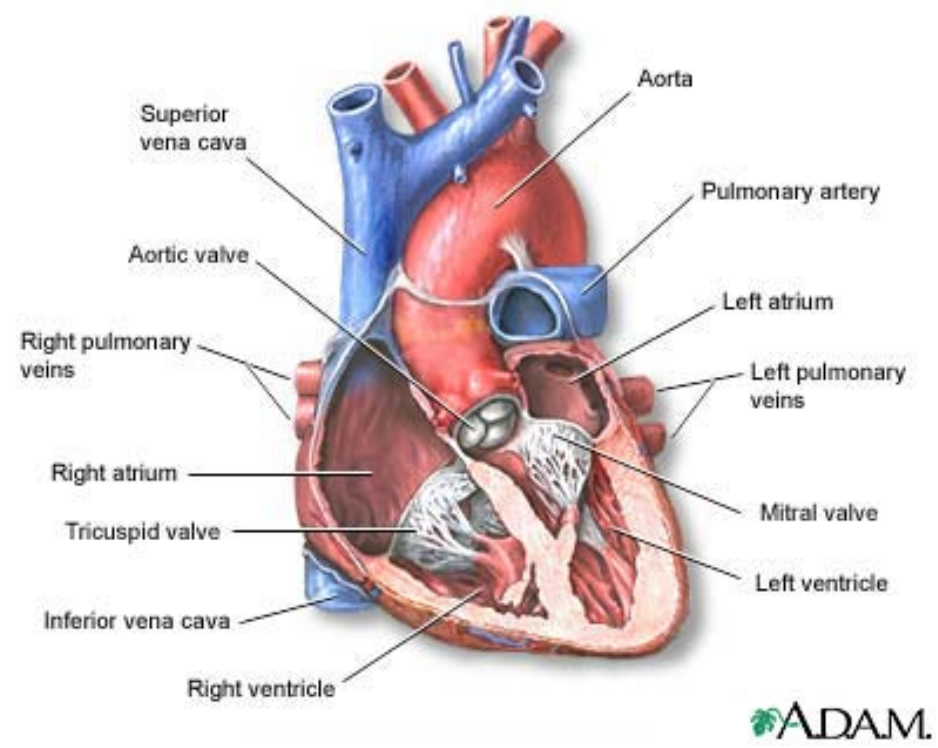

Figure 2.1: A cut-away view of the human heart and valves [9]

\section{The Cardiac Valves}

The cardiac valves are responsible for controlling the flow of blood through the heart. There are two pairs of valves, one pair located in the junction between each atria and ventricle, known as atrioventricular valves, and one pair located at the exit of each ventricle, known as semilunar valves. Semilunar valves operate in a passive manor, based purely on flow dynamics [II]. When flow rushes through a semilunar valve, a portion diverges into the chamber around the valve known as the sinus. This diverged flow forms a vortex before rejoining the main stream. When the ejection pressure reaches its peak, the flow begins to decelerate creating an adverse pressure gradient. As back-flow begins, vortical flow redevelops in the sinus chambers behind the leaflets causing them to shut [28].

Disease can interfere with the normal operation of the valves causing weak or hard leaflets. These abnormalities can affect the seal of the leaflets and allow fluid to leak back into the preceding chamber. This is known as regurgitation. In some cases, leaflets may not seal entirely and simply collapse back into the chamber, known as prolapse [24].

\section{Blood}

Blood facilitates the transport of oxygen and nutrients to tissues, as well as the removal of waste and carbon dioxide. In addition, the flow of warm blood from the core helps regulate body temperature. The transport of white blood cells also supports the body's immune response. The density of blood is slightly higher than that of water, between 1050 and $1060 \mathrm{~kg} / \mathrm{m}^{3}$ and the average person has between 4.5 


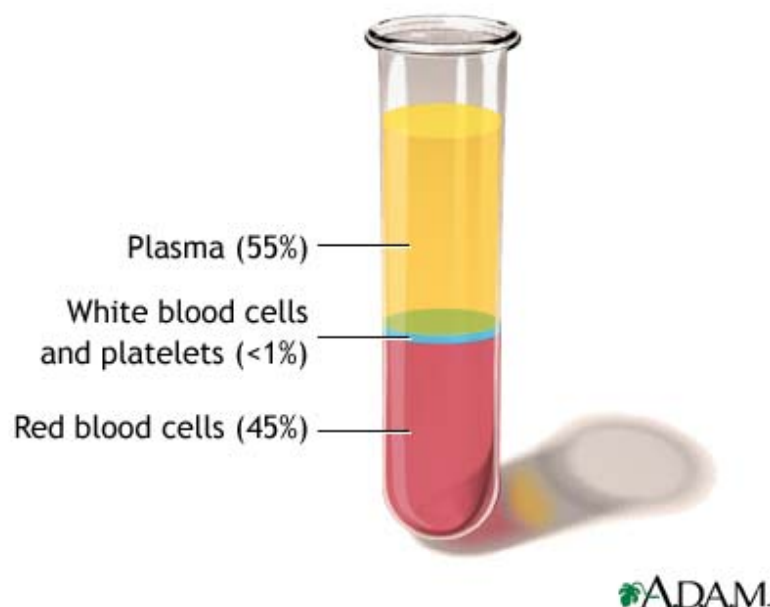

Figure 2.2: The three components of blood [].

to $6 \mathrm{~L}$ in their body [87]. As shown in Fig. [2.2, blood is comprised of three main components: plasma, white blood cells, platelets, and red blood cells. Depending on the kinematic status of the fluid, it can behave in a myriad of ways. Besides its multi-phase nature, another important property of blood from the perspective of fluid dynamics is its non-newtonian behaviour, discussed in section 2.3 .

\section{Systemic Vasculature}

The vasculature of the body plays an important roll in regulating hemodynamics by creating peripheral vascular resistance. In general, human vasculature is not rigid but highly deformable with the ability to dilate and contract to control blood pressure and volume. At a high level it can be subdivided into two segments, arterial and venous. Arteries are responsible for carrying oxygenated blood to body tissues. They can be further subdivided into narrower sections called arterioles and ultimately reduce to capillaries where cellular gas exchange occurs. The venous system is similar, however begins with the deoxygenated blood from the tissues and returns it to the heart where it is forwarded to the lungs (see Appendix [B.]) [6.3]. The vascular system also provides an important capacitive effect which can act to absorb some of the pulsatile energy from the flow [28].

\subsection{Human Response to Inertial Load}

When an individual is exposed to an an acceleration, the result is an increase (or decrease) in inertial load felt by the person. On earth, everyone is subjected to a standard gravity acceleration of $9.81 \mathrm{~m} / \mathrm{s}^{2}$, otherwise known as 1G. Depending on the axis the acceleration is applied, G-forces can be defined as either vertical $\mathrm{G}\left(G_{z}\right)$, in an axis running head-to-foot, lateral $\mathrm{G}\left(G_{y}\right)$, on a side-to-side axis, or in the 


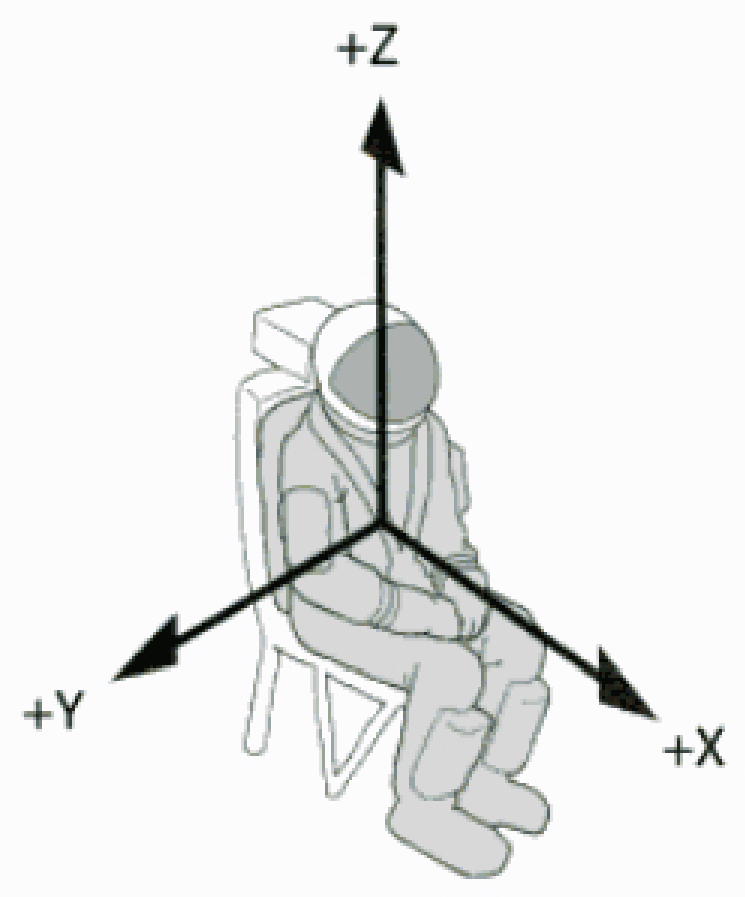

Figure 2.3: Gravitational force axis [22].

transverse $\mathrm{G}\left(G_{x}\right)$ direction, front-to-back.

The affect of increasing $\mathrm{G}$ on humans can be profound. Most often seen by pilots or astronauts, high G can restrict the cardiovascular system's ability to supply oxygen to the brain and vital organs resulting in a condition known as cerebral hypoxia. Fig. ए.] illustrates these symptoms including narrowing of vision (grey-out) progressing to a complete loss of vision (blackout), and ultimately loss-of-consciousness. Maintaining blood flow, and thus blood pressure is therefore key in managing cerebral hypoxia caused by an increase in $\mathrm{G}[22]$.

One can estimate relative blood pressure at various locations in the body through the use of the hydrostatic equation (Eqn. ए.]). This states that pressure in a column of fluid can be represented by the product of density, gravity, and the height of the fluid column

$$
p=\rho g z
$$

where $p$ is the internal pressure, $\rho$ is the fluid density, and $z$ is the height of the fluid column. If we consider $\rho$ to be the density of blood, roughly $1050 \mathrm{~kg} / \mathrm{m}^{3}$, and the standard acceleration due to gravity of $9.81 \mathrm{~m} / \mathrm{s}^{2}$, we can vary z to estimate the pressures at various vertical distances in the body, relative to the aortic valve $[22]$. For example, if the distance from the aortic valve to the brain is $40 \mathrm{~cm}$, the difference in hydrostatic pressure in that segment of the vascular system would be $p=(1050)(9.81)(0.40)=3605 \mathrm{~Pa}$. 


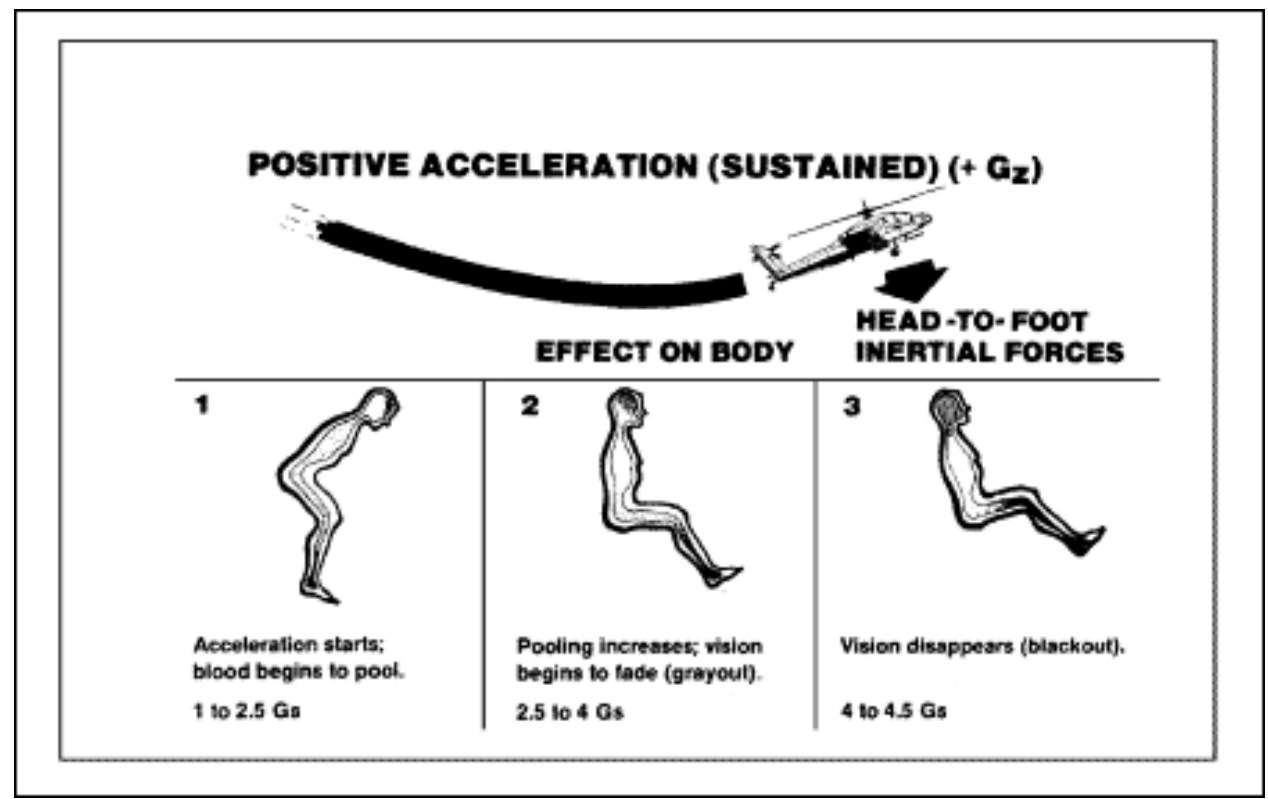

Figure 2.4: Effects of positive $\mathrm{G}$ acceleration in the longitudinal Z direction [4].

If this person was then subjected to an inertial load equivalent to 4 times standard gravity in the vertical direction, or $+4 \mathrm{G}_{z}$, the difference in hydrostatic pressure exerted by that column of fluid would increase to $16480 \mathrm{~Pa}$. If the body does not compensate for the increase in stress and maintains an average systolic pressure of $15998 \mathrm{~Pa}$, it is clear that cerebral flow will be compromised.

The body has various mechanisms for compensating for this reduction in perfusion including increasing heart rate, stroke volume, and systemic blood pressure (peripheral vascular resistance). However these mechanisms have upper limits and take time to react. The average upper limit for heart rate is generally 170 beats/min [22], whereas the highest safe systolic blood pressures is roughly $240 \mathrm{mmHg}$ [10]. Since between 6 to 9 seconds are required for the upper body compensatory mechanisms to activate, the rate of $\mathrm{G}$ onset also becomes a critical factor. Normally, the brain has a reserve time between 4 to 6 seconds before hypoxia sets in. As this is less than the time required for compensation, acceleration rates in excess of $0.2 \mathrm{G} / \mathrm{s}$ can cause complications if not assisted by external means such as anti-G suits or straining maneuvers which act to retain blood flow in the upper body [22].

\subsection{Fundamental Biofluid Dynamics}

The complex nature of blood flow creates unique challenges compared to fluid analysis in other engineering disciplines. Beginning with a simplified analytical baseline, one may assume the behaviour to be similar to steady flow through a tube. The most applicable starting point in this case is Poiseuille's Equation. Serendipitously, since Poiseuille was not only a physicist but also a physician, much of the 
development of his equation was actually based on modelling the circulatory system. The equation states that volumetric flow through a conduit increases by a power of 4 with increasing internal radius [57],

$$
Q=\frac{2 \pi\left(p_{1}-p_{2}\right)}{4 \mu L} \int_{0}^{R} r\left(R^{2}-r^{2}\right) d r
$$

where $p_{1}$ and $p_{2}$ represent pressure at each end of the tube, $\mu$ represents the fluid viscosity, $\mathrm{L}$ is the length of the pipe, $\mathrm{r}$ is the radius, and $\mathrm{R}$ the maximum radius. However there are many assumptions made in the development of this equation that make it inapplicable to cardiovascular flow in many cases. These assumptions require newtonian, homogeneous, and steady flow through a rigid vessel. In general the behaviour of the flow changes depending on the region of the body in question. Closer to the heart, the flow is highly pulsatile, although deep into the vasculature, it becomes steadier and characteristically non-newtonian. It is important to note that the Poiseuille assumptions are not adequately met in any of these circumstances, requiring the full Navier-Stokes equations for accurate analysis.

\section{Pulsatile Flow}

One of the most recognizable characteristics of the human body is the pulse. When the ventricles contract, the change in volume creates a pressure gradient that forces blood through the body. The pressure wave generated travels through the vasculature at a rate nearly 500 times the flow speed. This has many effects including changing the turbulent transition points and pressure distribution through the arteries. For example, when examining the flow field in the ascending aorta, one would imagine by the high instantaneous Reynolds number that the flow during systole would be highly turbulent. However, the strong flow acceleration actually has a stabilizing effect and delays the onset of turbulence until the flow begins to decelerate. The resulting instabilities are mainly due to the adverse pressure gradient accompanying the deceleration. In addition, the lower than expected levels of turbulence are also attributed to the short period of time that these pressure gradients are present. This reduces the time available for these disturbances to propagate to the entire flow field, resulting in low levels of turbulence in most healthy arteries [119].

In its simplest form, a relation for pulsatile flow can be developed assuming incompressible, fully developed flow in a rigid, circular tube,

$$
\rho \frac{\partial u}{\partial t}+\frac{1}{\rho} \frac{\partial p}{\partial x}=\mu\left(\frac{\partial^{2} u}{\partial r^{2}}+\frac{1}{r} \frac{\partial u}{\partial r}\right),
$$

where $\rho$ is the fluid density, $\mu$ is the viscosity, $p$ is the pressure, and $r$ is the radius from the centre of the artery. Examining the behaviour of Eqn. [2.3 can illuminate important qualitative aspects regarding the behaviour of pulsatile flows, useful in further computational analysis. For example, if we assume the time-dependant pressure to be a standard sinusoidal function, the rise and fall in pressure will drive a corresponding rise and fall in flow. If the frequency of the oscillations is low enough, the flow motion and pressure curve will be notably in phase. However as the frequency of the pulse rises, a phase difference 
between the flow motion and the pressure pulse will develop. This phase lag essentially acts to dampen the peak flow velocity from what it may achieve in a fully developed, steady Poiseuille flow of equivalent peak pressure [94]. Therefore it is important to expect some degree of overestimation when analyzing steady approximations to pulsatile flow. The higher the frequency of the driving pressure (heart rate), the higher the reduction in peak flow. It is also important to note that the pulse frequency affects the velocity profile as well as peak flow rates. As the frequency increases, the flow does not have enough time to develop completely, whereas at very low frequencies, in conditions that comply with the necessary assumptions, the flow can be adequately defined by Eqn. [2.2 alone [94].

Another disadvantage of the Poiseuille assumptions in an application to hemodynamics is the necessity of a rigid tube. In reality, the elastic nature of arteries allows the tissue to locally deform with the introduction of a pulse and return with a corresponding recoil. This generates a wave motion in the tissue, adding a radial component to the flow velocity, which also becomes dependent on the axial position $x$. For an accurate solution, the simulation must then be coupled to a structural constitutive model representing the behaviour of the tissue, further discussed in Section 4.3.3.

In normal engineering analysis, the Reynolds number, expressing the ratio of inertial to viscous forces is utilized to estimate the stability of the flow. However, it has been shown that the Reynolds number is not effective in conveying stability in pulsatile flow, largely because in highly pulsatile regions, the flow is rarely fully developed. Using the Reynolds number in these situations generally over predicts turbulence by a considerable degree [19]. Thus, for pulsatile flow, the dimensionless parameter known as the Wormersley number, $\alpha$, is commonly used:

$$
\alpha=\frac{d}{2} \sqrt{\frac{\rho \omega}{\mu}},
$$

where $\omega$ refers to the heart rate in radians per second, $\rho$ is the fluid density, $\mathrm{d}$ is the diameter of the artery, and $\mu$ is the fluid viscosity. The significance of the ratio is an evaluation of inertial to viscous forces, adjusted for unsteady flow. The range of $\alpha$ in a healthy person ranges from $10^{-3}$ downstream to roughly 20 in the aorta. Wormersley number values less than 1 generally indicate a form of quasi-steady flow in which Eqn. 2.2 could be employed. Careful evaluation of the Wormersley parameter can actually provide important information into velocity profiles and levels of forward flow [1.9].

Also essential for any numerical analysis is obtaining a mathematical representation for the pressure cycle. The most versatile approach is to develop an analytical function representing the pressure at any point in time. This can be accomplished using a Fourier series which allows the representation of any periodic function to be expressed as a superposition of sin and cosine functions. For example, a nine parameter Fourier series representing inlet pressure would have the form,

$$
p(t)=a_{0}+a_{1} \cos (t \omega)+b_{1} \sin (t \omega)+\ldots+b_{8} \sin (8 t \omega),
$$

where $a$ and $b$ are curve fit coefficients unique to the periodic function. This approach is attractive as it 
is easy to incorporate into a computational algorithm.

\section{Heterogeneous nature of blood}

The presence of red cells and platelets give blood very unique properties depending on its kinematic state. When blood is still, cells tend to coagulate, creating a three-dimensional lattice structure, allowing it to resist shear. This, in addition to the variable concentration of red blood cells (hematocrit) affects the blood viscosity and results in non-newtonian shear behaviour. Normally this is seen with a red blood cell concentration of over $10 \%$ [57]. The coagulative contribution to viscosity can sometimes take up to 30 seconds to develop. Therefore it is observed less in the highly pulsatile regions closer to the heart where the intense cyclic pressure waves break up these microstructures. Blood in these regions is subsequently assumed newtonian, although other physiologic factors can affect the validity of this assumption [42].

\subsection{Biosolid Mechanics}

\subsubsection{Arterial Structure}

A normal human artery is comprised of three main layers: the intima, media, and adventitia. Within each layer is a network of various biologically elastic materials. The most significant of these materials is elastin, and collagen. These molecules have vastly different properties, and therefore affect the structural mechanics of arteries in different ways. Fig. 2.5 illustrates the three-layered physical structure of a normal human artery [37].

\subsubsection{Elastin}

Elastin is a protein molecule that is found in many mammals and makes up a large portion of blood vessel walls. Mechanically, elastin is slightly viscoelastic however exhibits some of the most linearly elastic behavior in the body. The behavior of elastin is primarily a result of internal energy changes and entropy. The molecules are structured as long protein chains, which are always in motion due to thermal energy. Under strain, the molecular configurations change which creates a corresponding change in entropy. This resulting entropy change is manifested in elastic stress [27]. Therefore some preliminary inferences on the material behavior can be developed simply from thermodynamics. The shear modulus, $G$, can be related to the ideal gas constant, $R$, temperature, $T$, density, $\rho$, and average molecule weight of elastin, $M[27]$.

$$
G=\frac{\rho R T}{M}
$$




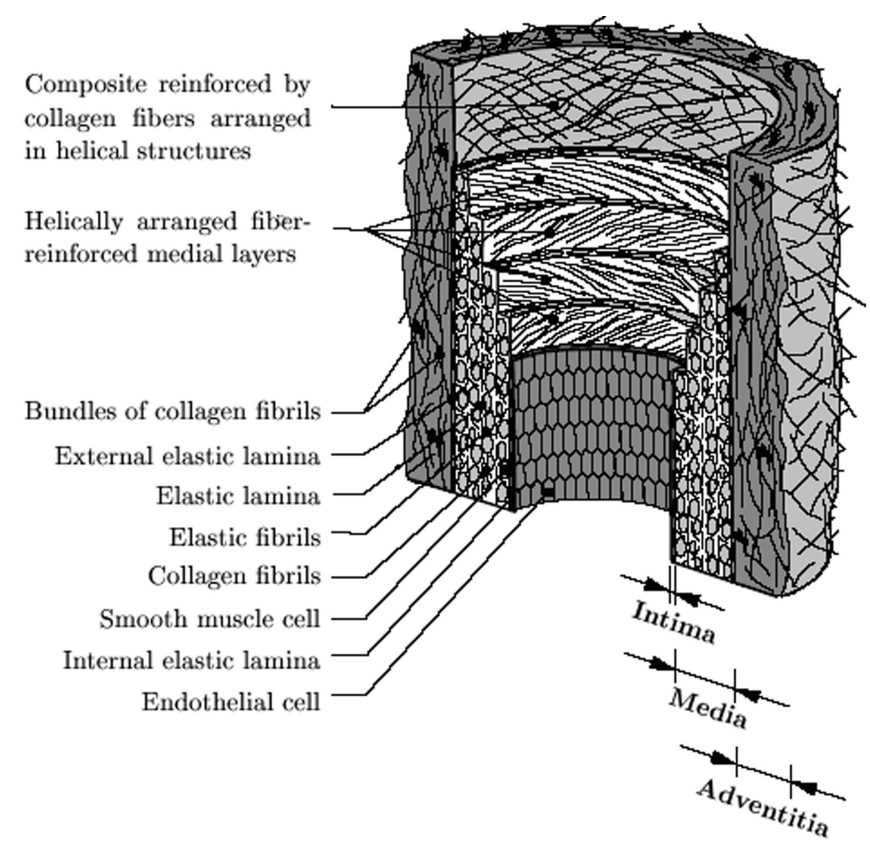

Figure 2.5: Physical structure of a normal human artery [37].

From this, the Young's modulus can be defined as,

$$
E=2(1-\nu) \frac{\rho R T}{M},
$$

where $\nu$ represents the Poisson ratio. The simplicity of Eqn. 2.7 creates an opportunity to embed the behaviour of elastin into a larger composite based model.

\subsubsection{Collagen}

Collagen is the primary load bearing structure in most vascular structures. It is mainly comprised of helically wound proteins in a structure similar to many long chain polymers. This structure is important when looking at the behavior of the material. Under small strains, these long protein chains remain coiled and do not support significant load. However, as the strain is increased and the molecules are stretched, they begin to support substantial stress. This phenomenon leads to the highly nonlinear behavior of human tissue. As in other composite materials, the orientation of these fibers is essential to the overall behaviour and response [27]. Table [2.4.3 provides a comparison of the mechanical properties of collagen and elastin. By examining the physical structure of these materials, it can be seen that a true constitutive representation of the aortic arch would involve a highly nonlinear and anisotropic model. 


\begin{tabular}{lll} 
Material & Youngs Modulus (MPa) & Tensile Strength (MPa) \\
\hline Collagen (axial) & 1000 & $50-100$ \\
Elastin & 0.6 & - \\
Aluminum (6061T6) [43] & 68900 & 310 \\
Femur Bone (longitudinal) & 20000 & - \\
\hline
\end{tabular}

Table 2.1: Biomaterial property comparison [28].

\subsection{Numerical Modelling of the Aorta and Aortic Arch}

Many attempts at modelling the solid and fluid regimes of the aorta numerically have been published with various levels of model fidelity. Early analysis involved the use of idealized geometries such as a simple curved conduit of constant radius. Taylor et al. [ㅍ] utilized a finite element approach to model unsteady pulsatile flow through an idealized model of the abdominal aorta. Their study focused on the application of numerical methods towards the prediction of wall shear stresses and their relevance to the development of atherosclerosis. With the development of higher accuracy medical imaging, numerical studies began to incorporate geometry with a larger degree of anatomic realism. In 2003, Leuprecht et al. [47] published a comparative study of blood flow in the ascending aorta between experimentally measured magnetic resonance imaging (MRI) data and a CFD based model with geometry developed from the MRTl data. Their showed the potential of such analysis and identified pitfalls associated with geometry based on medical imaging. A study by Shahcheraghi et al. [75] presented a three-dimensional, unsteady simulation of flow in the aortic arch based on geometry obtained from $\mathbb{C T}$ imaging. They used experimentally based pulsatile inlet conditions, and volumetric outlet conditions which proportion the flow through each outlet based on the model geometry. This study highlighted numerically, various flow behaviour such as skewed velocity profiles, retrograde, and helical flow patterns that have been previously observed in various experimental studies [45][26][56][53]. The study also presented a comparison of documented atherosclerotic legions in zones of maximum or minimum pressure and shear stress, suggesting that such phenomena could be effectively examined numerically. Subsequent research into effective boundary conditions emerged with the intent of incorporating the influence of the downstream network of bifurcating arteries and veins. Various approaches were developed including resistive

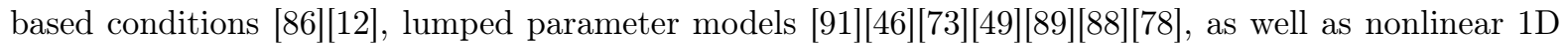
Navier-Stokes based models [5.9][주][10].

A similar history in tissue modelling of the aortic arch using finite element methods can be traced. Investigations of stress analysis in the cardiovascular system began as early as 1969 [50][31]. Early models utilized 1D axisymmetric models designed to simulate the response of cardiac tissue [64]. Since these early inquiries, much attention has been devoted towards developing constitutive models of biological tissue appropriate for use in finite element analysis. Various models [35][39] [38][34] and frameworks [37] have been developed for tissue response incorporating differing levels of fidelity. Recent studies in the region of the abdominal aorta and aortic arch have focused on estimating the development of atherosclerotic plaque or properties of aneurysms. In 2004, Venkatasubramaniam et al. [8.5] generated 3D based geometries of 
27 patients with abdominal aortic aneurysms using CT based imagery. Another application of analysis based upon 3D $\mathbb{M R}$ l reconstructions was published by Beller et al. [14] in which the superior portion of the aortic arch was examined in regards to the affects of wall stress on aortic insufficiency. Other applications included the investigation of root movement in aortic dissection [13].

Fluid-structure investigations have also been attempted recently, with the majority of applications focused on predicting pathologies such as plaque and aneurysm formation. A study in 2003 by De Hart et al. [23] examined the operation of the aortic valve leaflets using a Lagrange multiplier based fictitious domain method in conjunction with unsteady fluid and linear isotropic solid properties. This solution was further investigated in a study on the combined use of arbitrary Lagrangian-Eularian (ALE)] and fictitious domain (FD) methods in cardiovascular analysis. Other applications of Huid-structure interaction (FST) modelling include heart valve dynamics [83] [40] [18], aneurysm modelling [92], and ventricular dynamics [20]. There have also been published studies into ESTl modelling of the aortic arch. Gao, Watanabe, and Matsuzawa used a loosely coupled EST model to investigate stress behaviour in a layered aortic arch model under pulsatile blood flow [30]. Although this model idealized the aortic arch to simple U shaped tube, it showcased an expansion of constitutive models to include layered models in an EST analysis. Bazilevs et al. [12] presented an [ESTl investigation of the aortic arch incorporating a left ventricular assist device (LVAD). In this investigation, CT based imagery was used to generate the model geometry along with experimentally based inlet and resistive based outlet boundary conditions.

Nearly all presented studies have a common application in simulating pathophysiologic phenomena, but not necessarily examining the effects of external environments or applied loads.

\subsection{Analysis of Inertial Loading}

Historically, research into the effects of $\mathrm{G}$ forces on the body has been primarily qualitative through the use of human centrifuges. Individuals are subjected to increasing $\mathrm{G}$ until physiologic effects such as vision narrowing or unconsciousness are felt. Although this is an effective practical tool for determining acceleration tolerance, it does not reveal any information as to the degree of physiologic detriment. Primitive quantitative efforts generally included the measurement of basic body vital signs including heart rate, blood pressure, and respiration rate. Some studies have expanded to examine the effects of G on the electrical activity of the heart by examining the electrocardiogram (ECG) of patients undergoing centrifugal acceleration. In the late 1950's, Wood et al. [9:3] under contract with the United States Air Force began efforts to quantify changes in cardiac output and related physiologic variables under the influence of inertial load. Their research involved catheterization of the aortic arch and vena cava proximal to the right atrium. Subjects were exposed to forward and headward accelerations while physiologic parameters were monitored. The results of the study indicated a $22 \%$ reduction in cardiac output under $4 \mathrm{G}$ headward acceleration.

Recent studies include the use of noninvasive measurement modalities, providing an avenue for deeper 
exploration of the flow field or investigation into regions where catheterization is impractical. One study included the use of transcranial doppler ultrasound imaging to record the velocity of flow through the middle cerebral artery [ 82$]$. Such a technique shows immense potential due to the portability and noninvasive nature of the device.

Although experimental measurement is the standard method of determining an individual's G tolerance and flight readiness, various analytical models for predicting cardiovascular response have been developed. In the late 1960's, several lumped parameter models were developed with the intent of simulating the effects of acceleration on physiologic parameters such as cardiac output and blood pressure. In 1968, Snyder and Rideout [77] developed a comprehensive lumped parameter model of the systemic arterial system translating all major arterial branches into capacitive and resistive analogs (Fig. [2.6]). By calculating the correct constants for each branch, the complete model could then be used to determine flow velocity and pressure at any point.

Similar models were also developed for pulmonary circulation [69] and venous return. In 1973, Green and Miller [33] developed a lumped parameter model of the venous system specifically for simulating acceleration stress. This approach is of particular interest as the importance of maintaing adequate venus return is crucial under larger $\mathrm{G}$ forces where pooling of blood in the legs prevents blood from returning to the heart.

Although various analytical models have been developed to simulate cardiovascular response under inertial load, to date, no published data using CFD or FEM techniques has been found. 


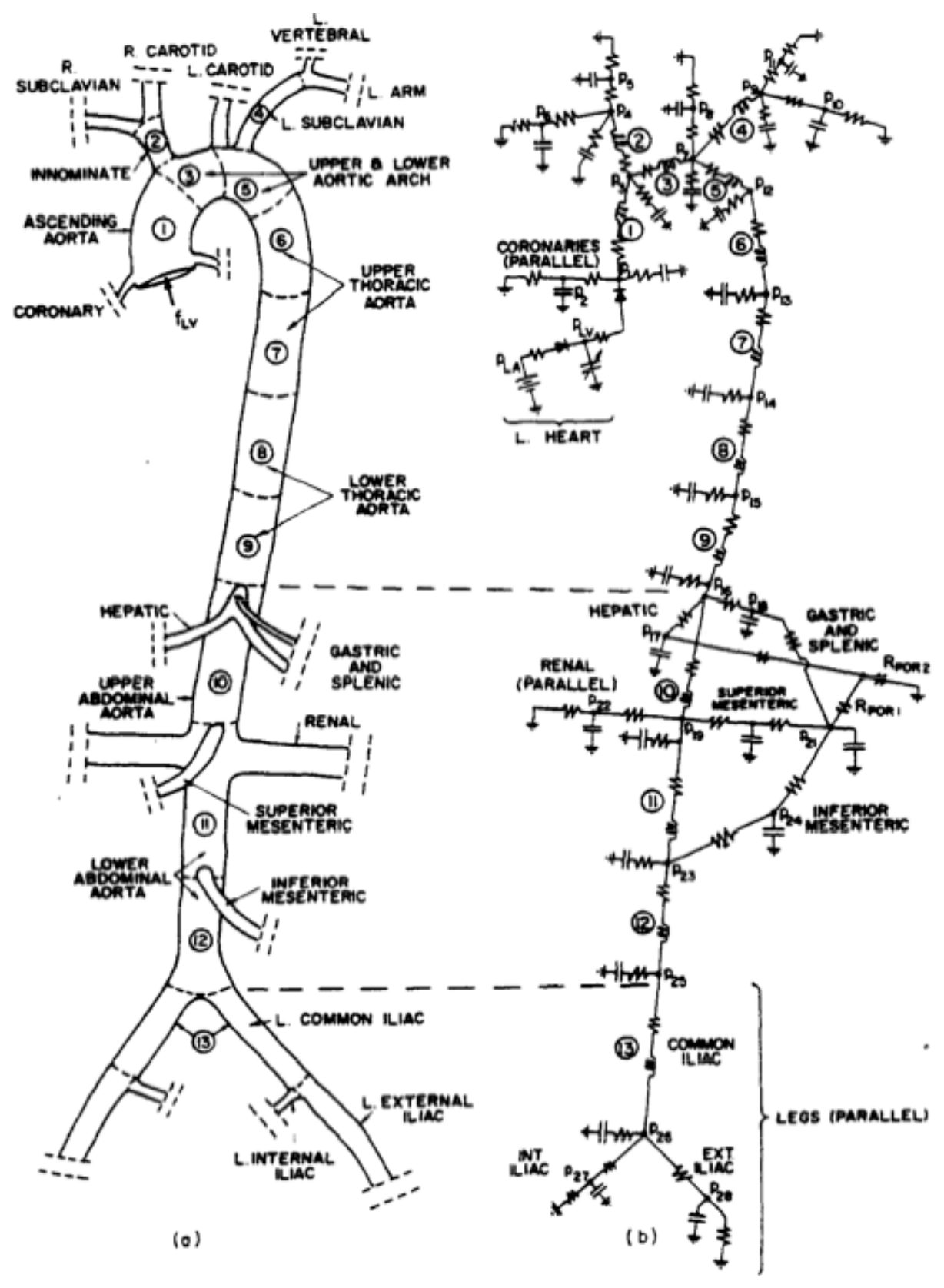

Figure 2.6: Diagram of the systemic arterial tree model developed by Snyder and Rideout, 1968 [7]]. 



\section{Chapter 3}

\section{Geometry and Mesh Generation}

\subsection{Overview of Geometry Generation}

Developing computational models of biological structures presents unique challenges with regards to obtaining a suitable geometry. Structures such as those found in the body tend to be rich with organic nuances and features that are often ignored when recreated using computer aided design (CAD). Therefore much research has been conducted into using medical imaging technologies as a conduit for creating accurate and anatomically correct geometry for use in engineering numerical analysis. Several medical imaging modalities exist for creating a three-dimensional representation of an anatomic structure, although the process has yet to become trivial.

\subsubsection{Computed Tomography (CT) Based Modelling}

CT is an extension of standard X-ray technology that allows a series of two-dimensional images to be stitched together to generate a three-dimensional model. A CT scanner uses an X-ray emitter and detector mounted on a rotating gantry which revolves around the body producing a cross-sectional "slice". As X-ray absorption varies with tissue density, the gradient of this absorption can be used to generate an image, mapping the absorption to pixel contrast on a grey scale. By obtaining successive cross-sections, advancing along the longitudinal axis of the body, each slice is interpolated with the next to produce the final $3 \mathrm{D}$ representation [1]7].

CD imaging has certain advantages over other modalities as it may provide a higher pixel contrast between regions of varying density. This can be advantageous when imaging bone, or searching for regions of blood. The higher contrast can also be advantageous when developing numerical models, making it simpler for a computer algorithm to detect the anatomic structure in question. 


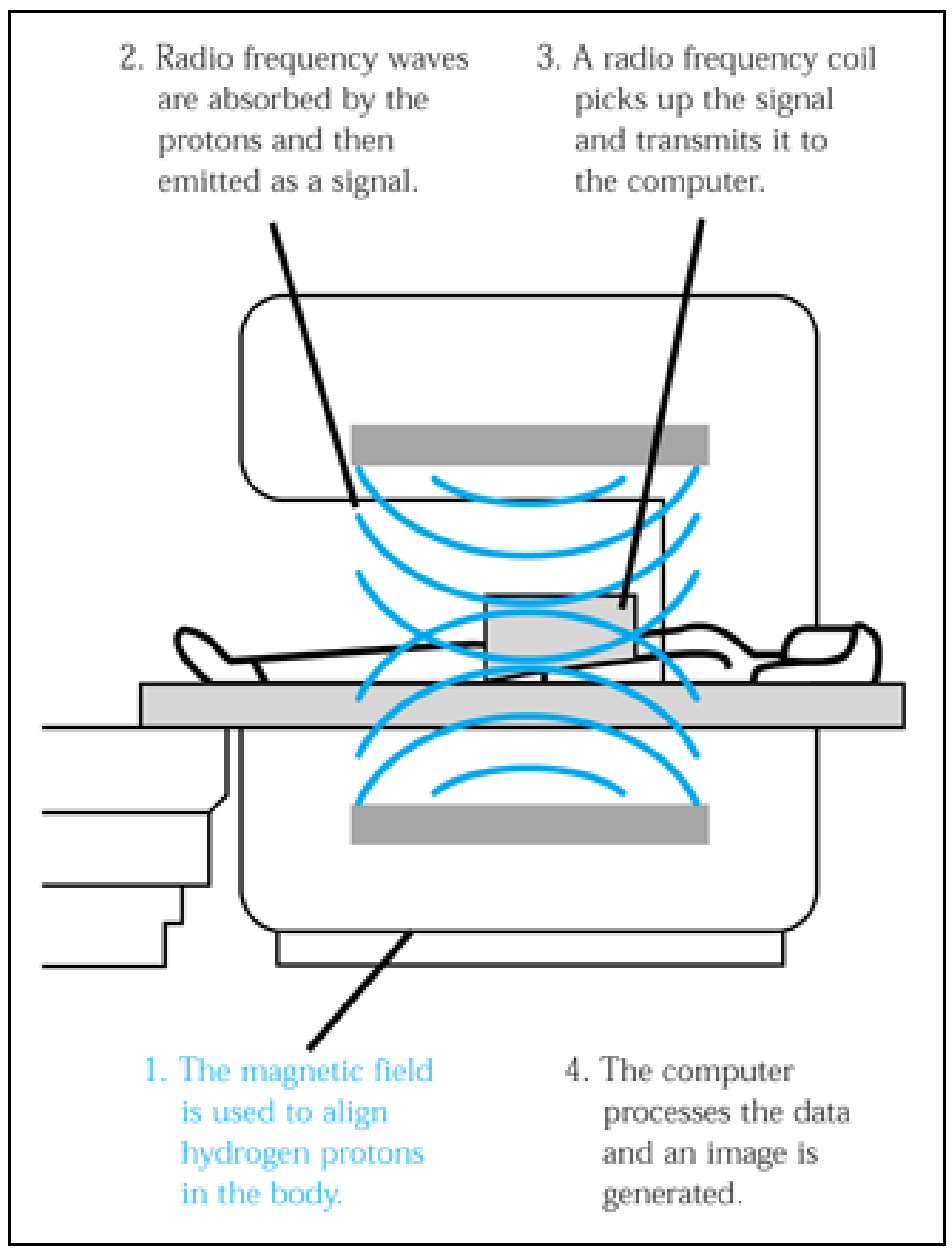

Figure 3.1: An illustration of image acquisition using MRI []].

\subsubsection{Magnetic Resonance Imaging (MRI)}

Magnetic resonance imaging has emerged as a popular imaging choice in health care. Unlike $\mathbf{C D}$, $\mathbb{\text { MKW }}$ uses no X-ray radiation to produce an image, making it safer for use with some patients. MRD uses a combination of strong magnetic fields and a radio wave transducer to generate highly detailed images of bones and soft tissues. The patient is placed in the center of a large superconducting magnet with a strength normally between 1- 3 Tesla (T). This causes the magnetic moment (spin) of the hydrogen atoms to align. A radio pulse is then sent towards the region in question. The hydrogen atoms absorb the energy from the pulse causing the spin to change. This new resonant frequency is detected by the sensor and converted into a cross sectional image showing the density of the tissue (Fig. [.]) [16]. Generally, पRR can produce a more detailed image of soft tissue structures than $\mathbb{C D}$, although the contrast between structures may be lower. This may cause difficulty in applying automated reconstruction algorithms. Nonetheless, $\mathrm{MRT}$ remains an important tool for modelling anatomic structures. 


\subsubsection{Segmentation and Model Development}

Once a series of images are obtained representing the anatomic structure in question, an algorithm must be employed to interpolate these images in three-dimensions, and if necessary extract the specific feature in question. This process is known as segmentation.

For the presented research, the objective was to develop an accurate model of the aortic arch. A CD scan of the thoracic chest cavity was obtained from a publicly available imaging database [6]]. The data set contained several hundred cross sectional slices beginning near the diaphragm, extending to the neck in $1 \mathrm{~mm}$ intervals. An algorithm was then used to extract only the aortic arch from the entirety of the chest cavity.

First, the 2D cross-sections are analyzed for regions of high contrast gradients which indicate a border between one structure and another, for example the aorta and lung. A curve is then defined around all high contrast regions. The result of this boundary identification is a series of curves representing all the anatomic structures in each slice. With a given distance between slices of $1 \mathrm{~mm}$, the curves are then joined using a triangular mesh. Once this process is complete, a triangulated surface mesh is formed providing a 3D representation of the anatomic body. Specific structures can be extracted by imposing boundaries or regions-of-interest on each 2D slice, limiting the region where the boundary identification takes place (Fig. [2). Although this resulting tessellated surface (Fig. B.3) can in theory be used for numerical modelling, further processing is generally necessary to advance the model to a form conducive to meshing operations and boundary definition. When such post-processing is required, a problem of compatibility can emerge in performing operations on a tessellated surface. Most CAD software is developed to operate on Non-Uniform Rational Basis Splines (NURBS) based geometry. Unfortunately the process of converting a complex tessellated surface into a NURBS based representation is not trivial. The approach used in this research analyzed the contours of tessellated regions and merged like regions into a simplified NURBS representation. The result is a collection of NURBS "patches", stitched together to create a full NURBS representation (Fig. B.4). This was accomplished using the software package 3-Matic (Materialize, Ann Arbour, MI). Once the NURBS based model was obtained, minor smoothing operations were performed to produce a consistent arterial wall. At all boundaries a flat plane was also imposed to aid in defining and meshing the boundaries. These steps greatly simplify the meshing process allowing compatibility with commercial meshing algorithms and solvers.

\subsubsection{Considerations for Medical Imaging in Engineering Analysis}

Although modern medical imaging has created a myriad of possibilities in engineering analysis, there are several considerations that should be regarded for a valid solution. As the use of medical imaging such as $\mathbb{C D}$ and $\mathbb{M R}$ l for geometry generation became more popular, the accuracy of their use has also been scrutinized [54][5]]. Research by Moore et al. [51] emphasized that variations in spatial resolution and reconstruction techniques can propagate into large errors in computed values. Techniques such as mesh 


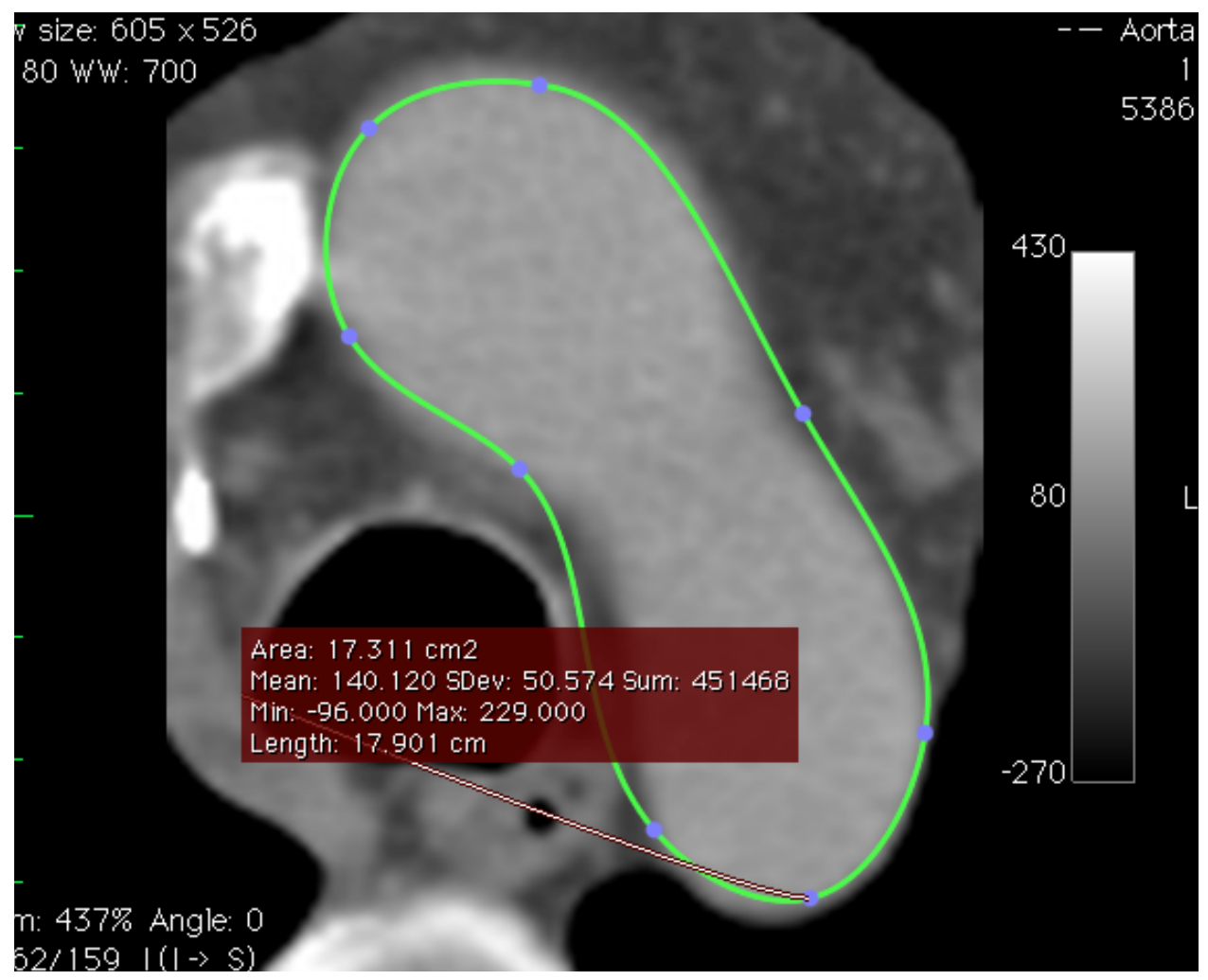

Figure 3.2: CT scan segmentation of the superior aortic arch.

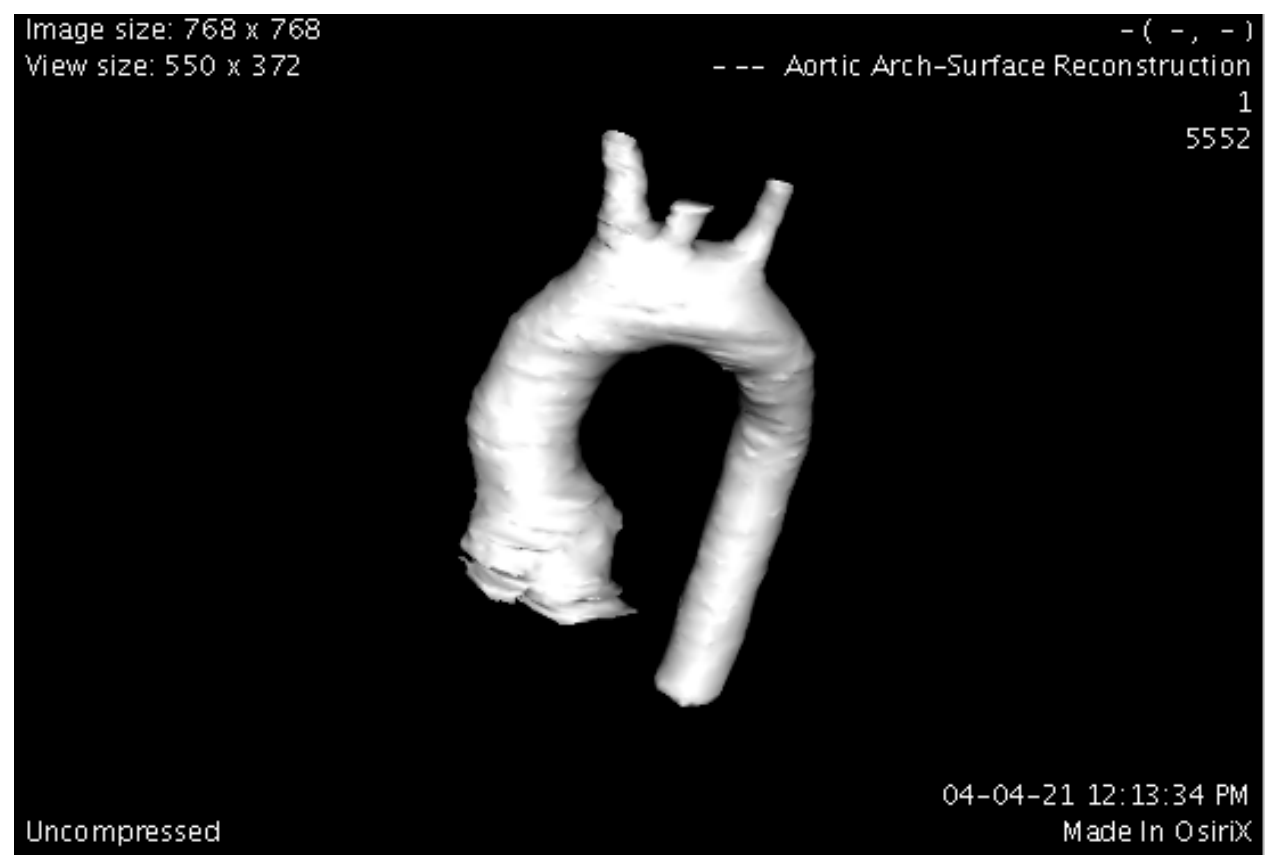

Figure 3.3: Rough surface reconstruction prior to smoothing operations. 


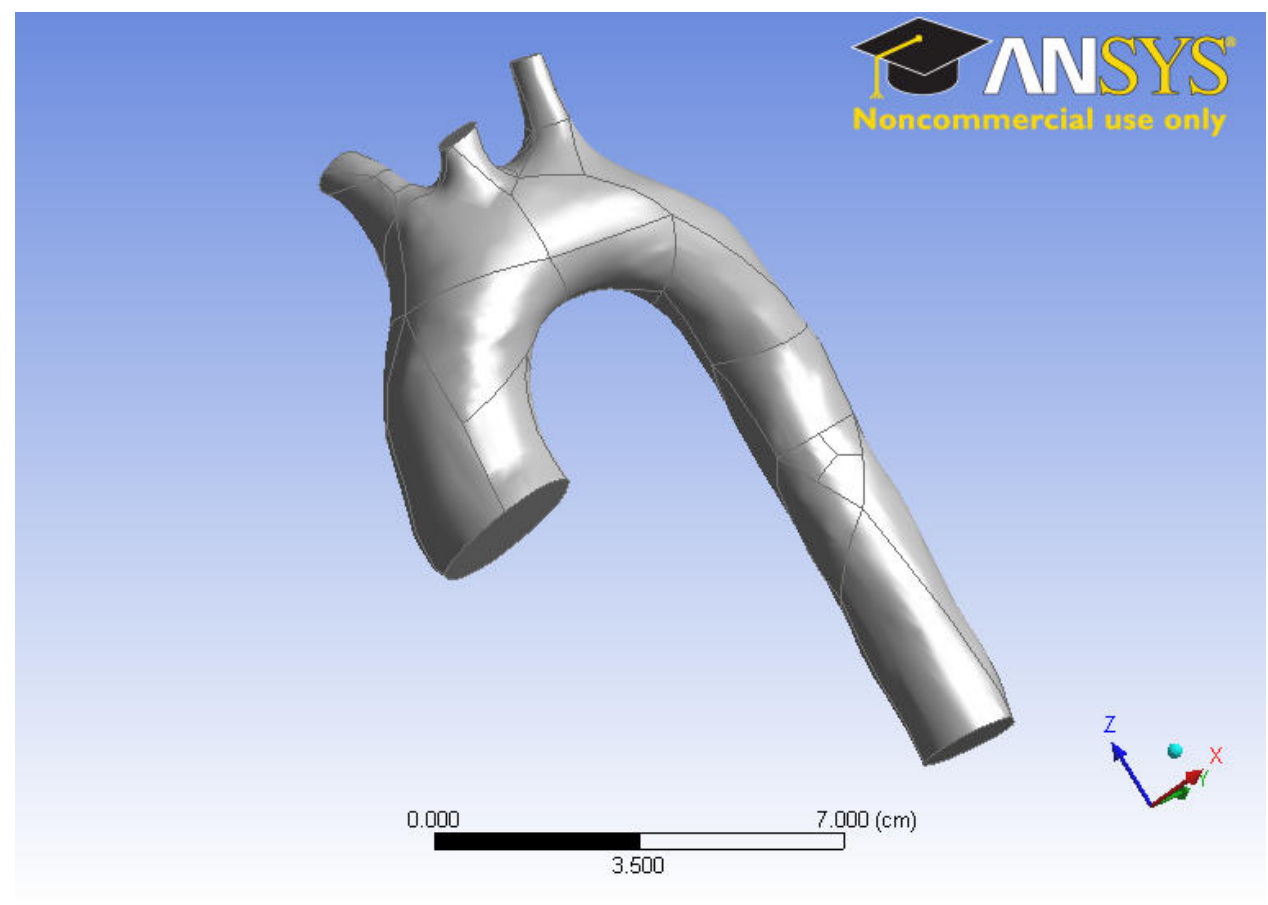

Figure 3.4: Completed non-uniform rational basis splines (NURBS) based model.

smoothing markedly reduced such errors and was recommended for analysis based on MR imaging. Such error is more important in the context of a patient specific analysis where the accuracy of the model geometry is paramount. In the presented case, the geometry is considered to be representative of the general shape and not an exact recreation of the original. It is likely that any error arising from image acquisition and segmentation will be similar to the natural variation of the aorta from one individual to another.

\subsection{Meshing Overview}

The organic shape and patched NURBS surface can create an interesting challenge with respect to meshing. A properly refined mesh should be fine enough to resolve the physics in the model with adequate accuracy, although not overly dense as to impede efficient computation. The fluid-structure approach taken in this research required two separate meshes to be constructed, for both the fluid and solid regimes. This is advantageous allowing the most appropriate meshing techniques and elements to be used to represent the solid and fluid mechanics. 


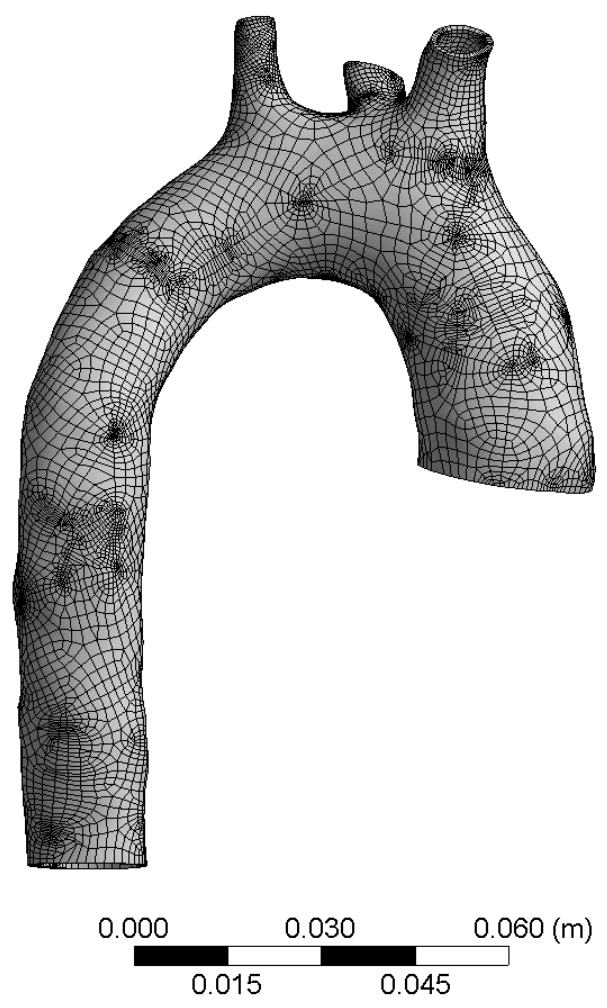

Figure 3.5: Localized mesh sizing errors due to $\mathrm{NURBS}$ patch topology.

\subsubsection{Solid Meshing}

In this investigation, the wall of the aortic arch must be modelled to accurately reflect the stress, strain, and deformation throughout the cardiac cycle. The forces on the wall are created primarily by the pressure inside the aorta, which is defined through the solution of the CFD model. There is also an inertial load applied globally to the system which will induce stress. As the wall forms a fluid-solid coupling region, the surface mesh on the wall should be a similar geometry discretization as the surface CFD mesh.

Several meshing strategies exist in modern meshing software. Many such schemes also utilize advanced sizing functions that automatically determine ideal sizing parameters based on contours, volumes, etc. It is important to consider however that especially in the case of a patched NURBS surface, many algorithms such as the advancing front method can misinterpret the patch structure and create localized, dense mesh regions that are not necessary or advantageous (Fig. 3.5 ). Furthermore, these regions add to the computational cost of the simulation. For this reason, a simplified quad meshing scheme was used for all solid meshing (Fig. B.6)). This ignored the patched nature of the underlying geometry and provided an acceptable, uniform grid. The final mesh contained 11,717 elements. 


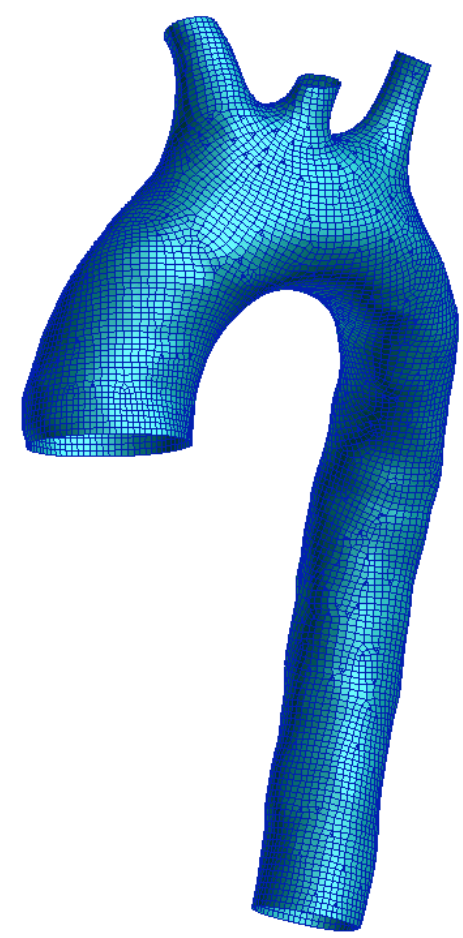

Figure 3.6: Overview of the quadrilateral solid mesh.

\subsubsection{Fluid Meshing}

The fluid regime required special care to ensure that characteristic flow behaviour such as helicity and boundary layer oscillations could be adequately resolved by the governing equations. This dictated the need for careful meshing of the boundary layer region. To accurately resolve the boundary layer, seven inflated prism layers were used with an expansion rate of 1.2 to a maximum thickness of $1 \mathrm{~mm}$ (Fig. B.7). This boundary layer thickness, $\delta$, was approximated using the Blausius solution for a flat plate (Eqn. [. [. $)$ [72] which models the thickness as a ratio of position, $x$, and the square root of Reynolds number, Re. This method was chosen for its simplicity although other methods may provide a more accurate initial reference.

$$
\delta(x)=\frac{5.0 x}{\sqrt{R e_{x}}}
$$

The volume of the arch was meshed using tetrahedral elements (Fig. 3.8), while the surface was meshed using a Delauny triangulation scheme. The surface mesh sizing was designed to be comparable to the solid mesh sizing to reduce interpolation error in the fluid-solid interface. 


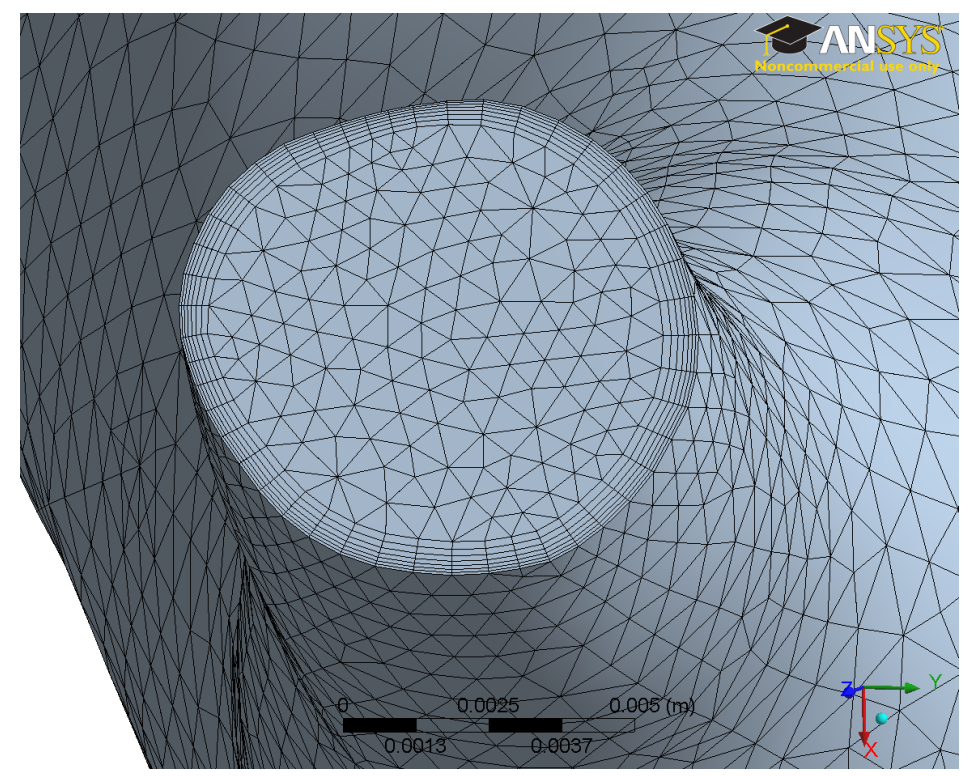

Figure 3.7: Closeup of inflated boundary layer near the left subclavian artery boundary.

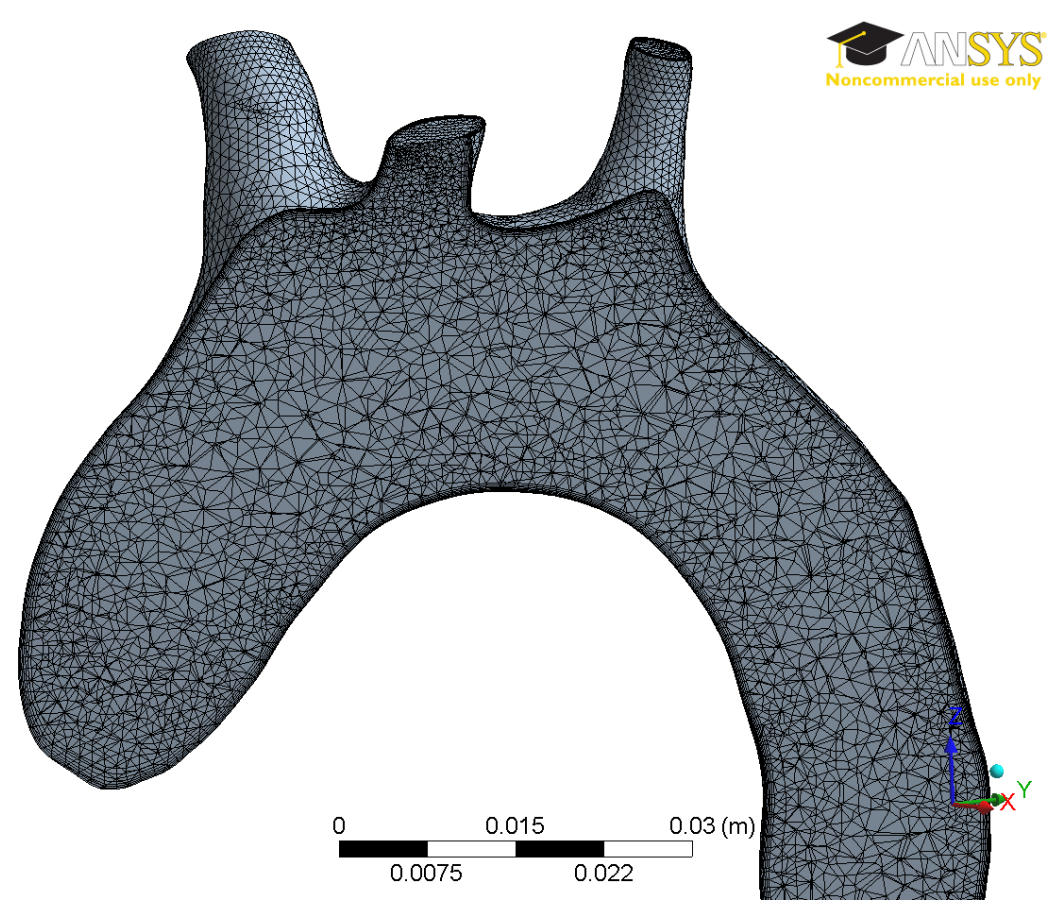

Figure 3.8: Section view of final fluid mesh. 


\section{Chapter 4}

\section{Methodology}

\subsection{CFD Solution}

\subsubsection{Inlet Boundary Conditions}

A cornerstone of this simulation is the ability to recreate accurate flow conditions in the aortic arch. Much of this fidelity is driven by the flow at the inlet of the aorta. In reality, flow at the inlet is generated by the contraction of left ventricle which forces blood through the aortic valve and into the ascending aorta. Once the ventricular pulse has propagated through the aorta, decelerating flow behind the pulse creates an adverse pressure gradient which forces the aortic valve shut and prevents any back flow of blood into the left ventricle. This valve operation is important to recreate numerically especially in the context of inertial loading, as the increase in hydrostatic load will increase the tendency for back flow through the inlet.

Generating an explicit model representing the characteristics and dynamics of left ventricular outflow is beyond the scope of this thesis. Instead, experimental velocity data obtained through hot-film anemometry of a human subject was used to define an inlet boundary condition distal to the valve. Using these experimental values, an analytical relation was developed with the use of a Fourier series analysis. Using a eight-parameter Fourier analysis in the software package MATLAB (Mathworks Inc.), the following nonlinear velocity relation for a plug flow profile in the ascending aorta was obtained using experimental results from Nichols and O'Rourke [57].

$$
v(t)=a_{0}+a_{1} \cos (t \omega)+b_{1} \sin (t \omega)+\ldots+b_{6} \sin (6 t \omega) .
$$

In Eqn. 4.], $v$ represents inlet velocity, $a$ and $b$ represent curve fit parameters, and $t$ represents the simulation time. Adjusting $\omega$ changes the frequency of the wave thereby allowing control over the 


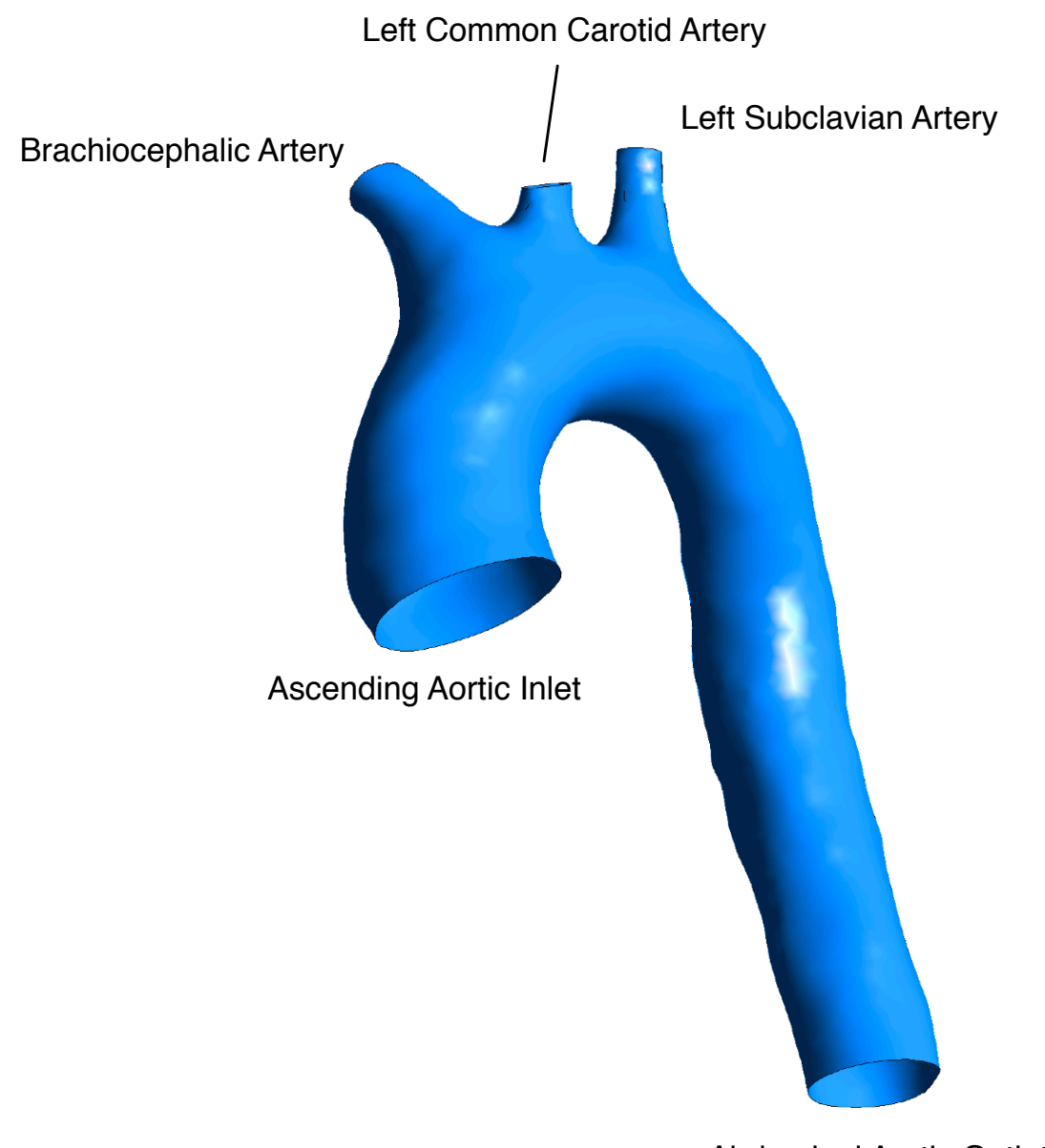

Abdominal Aortic Outlet

Figure 4.1: Diagram of CFD boundary conditions.

effective "heart rate" of the simulation. Detailed coefficients for this relation are provided in Appendix 因. By defining the velocity explicitly at the boundary, back flow through the inlet is prevented, imitating the operation of the aortic valve. Future models could incorporate more detailed characteristics of ventricular outflow including cardiac contractility and volume variations. Incorporating a model of the aortic valve would also increase the fidelity of the analysis as it is possible that increasing hydrostatic load on the leaflets could cause valve leakage or regurgitation. This may substantially alter the flow the arch and is worth further investigation.

\subsubsection{Outlet Boundary Conditions}

The aortic arch segment in this study contains four outlets, three ascending arteries at the top of the arch, and the outlet to the abdominal aorta (Fig. 1. (d). The conditions at each exit not only depend on 


\begin{tabular}{lll}
$C_{a}$ & Value & Location \\
\hline$C_{1}$ & $4.44 \times 10^{7}$ & Brachiocephalic Artery \\
$C_{2}$ & $3.60 \times 10^{7}$ & Left Common Carotid Artery \\
$C_{3}$ & $6.51 \times 10^{7}$ & Left Subclavian Artery \\
$C_{4}$ & $6.00 \times 10^{6}$ & Abdominal Aorta
\end{tabular}

Table 4.1: Resistive boundary coefficients.

the upstream conditions, but on the peripheral vascular resistance created by the downstream network of blood vessels. In addition, an appropriate boundary condition should be able to incorporate the pressure reflections and bifurcations present upstream and downstream. Three general techniques have been developed to approximate boundary conditions in biomedical flows.

\section{Resistive Boundary Conditions}

A resistive boundary condition lumps all the effects of the downstream vasculature into one time invariant parameter. The pressure at the outlet face $\delta \Omega$, can be described by Eqn. 1.2 [12]

$$
p=C_{a} \int_{\delta \Omega} \mathbf{v} \cdot \mathbf{n} d A,
$$

where $C_{a}$ represents the specific constant of peripheral vascular resistance for each outlet (Table 4.d). The integral of $\mathbf{v} \cdot \mathbf{n}$ is the volumetric flow rate across the boundary. Therefore the applied resistance is proportional to the flow rate.

Although this condition is the simplest to integrate into a numerical analysis, it lacks some important fidelity. The most problematic assumption is that it is time invariant. Therefore it is unable to simulate the effects of downstream wave propagation abundant in a pulsatile flow.

For comparison, a CFD analysis using resistive boundary conditions was performed using parameters

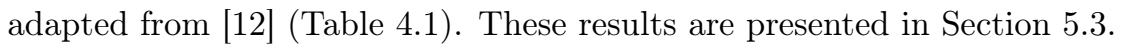

\section{Impedance Based Boundary Conditions}

An impedance based condition, like the electrical analog, is similar in action to resistance, although it is time-dependant. This allows important wave characteristics to be included in the system. One of the most common approaches to impedance based modelling is in the form of lumped parameter models. These treat each parameter as a black box and avoid implicitly solving any governing equations. Most lumped parameter models use some form of electrical analog. In this case, flow rates can be controlled through the action of a resistor while tissue compliance is modelled through a capacitor. Varying the number of elements, series-parallel connectivity, and component coefficient values allows the lumped parameter model to be adjusted to the proper behaviour. One of the most popular models is the 


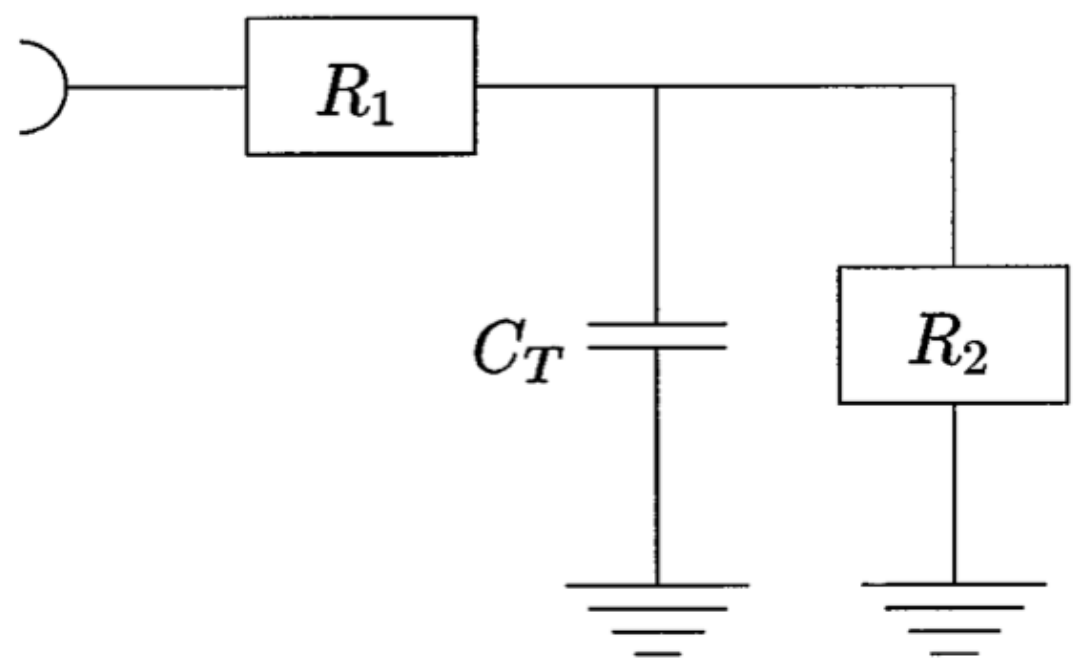

Figure 4.2: Three parameter windkessel model [5.9].

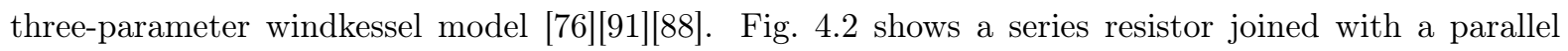
resistor-capacitor group. The sum of resistors $R_{1}$ and $R_{2}$ represent the total vascular resistance while $C_{T}$ represents the elasticity of the artery wall [5.9]. For this model to be of use however, coefficients for each component must either be experimentally determined or otherwise calculated. For a more in depth discussion of calculating parameters, the reader is referred to work by Shim et al. [76].

The main disadvantage of such models, is that although the resulting condition varies with time, the use of lumped parameters restricts the ability to simulate wave propagation effects [5.9].

\section{Structured Tree Models}

An interesting approach presented by Olufsen et al. [60] separates arteries into large and small sizes. Larger arteries are simulated using nonlinear Navier-Stokes equations, while the smaller arteries and capillaries are grouped into structured "trees" (Fig. 4.31). These trees are approximated using a hydrodynamic model based on a linear axisymmetric derivative of the Navier-Stokes equations. The linear equations are used in the frequency domain to estimate the impedance at the root of each vessel which is subsequently summed based on the geometry of an asymmetric tree of $N$ generations.

As this model is based on a variant of the governing equations, it provides the ability to simulate pressure-flow phase behaviour as well as wave propagation throughout the tree. This however is met with substantially larger computational cost and complexity by introducing additional time dependant equations that must be solved implicitly alongside the main solution. 


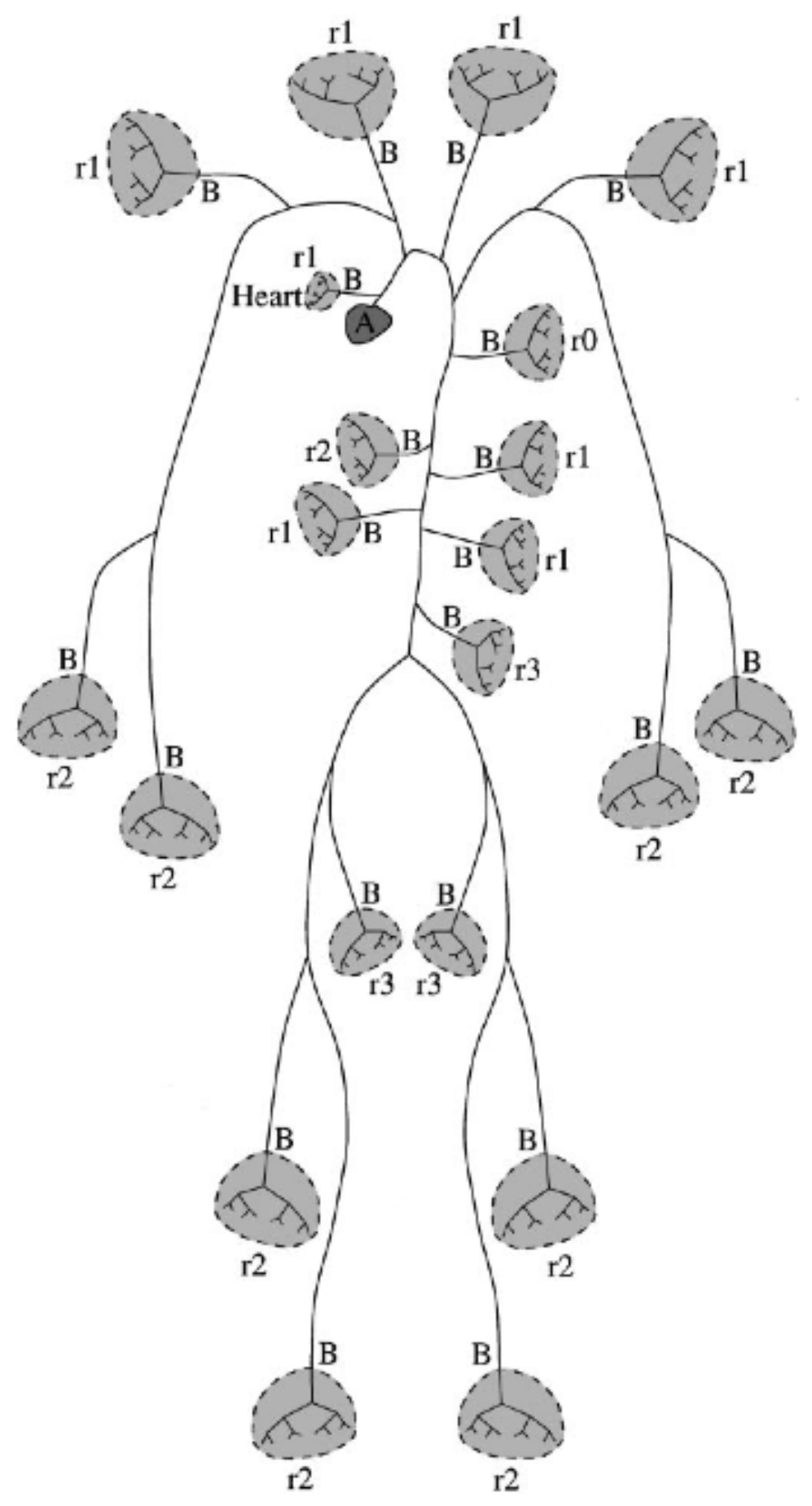

Figure 4.3: Olufsen structured tree model [59]. 


\begin{tabular}{ll} 
Property & Value \\
\hline Blood density, $\rho$ & $1060 \mathrm{~kg} / \mathrm{m}^{3}$ \\
Viscosity, $\mu$ & $0.0035 \mathrm{~kg} / \mathrm{ms}$ \\
Inlet pressure (steady) & $16 \mathrm{kPa}$ \\
Outlet pressures (steady) & $10.665 \mathrm{kPa}$ \\
Time step size (unsteady) & $0.01 \mathrm{~s}$ \\
Time steps (unsteady) & 200
\end{tabular}

Table 4.2: Steady and unsteady CFD simulation parameters.

\subsubsection{Solution Parameters}

The solver chosen for all CFD analysis was FLUENT (ANSYS Inc.). This decision was based on the reputation of the code in solving low Reynolds number internal flows as well as the ability to meet all simulation criteria. A pressure based solver was used and all discretization was second order in space and time. Momentum was simulated using a second order upwinding scheme.

The standard $k-\omega$ turbulence model was used as it has been shown to provide a good approximation to aortic flow [44][70][84]. This model uses the Boussinesq hypothesis, relating the Reynolds stresses to the mean velocity gradients. The model implemented in FLUENT is based upon the standard Wilcox $\mathrm{k}-\omega$ model and is optimized for low-Reynolds-number, wall bounded flow making it most applicable to the aortic flow being examined. Since the flow in the aortic arch is highly pulsatile, the assumption of newtonian behaviour was made, justified by the research of Ghalichi et. al. [25] [44]. Additional solution parameters are highlighted in Table 4.2 .

\subsection{Finite Element Solution}

\subsubsection{Linear Constitutive Models}

\section{Elastic Isotropic}

The simplest model of material behaviour is one where stress is linearly proportional to strain. Although this elastic behaviour does not necessarily coincide with the histology of arterial tissue, the use of the assumption provides a fundamental starting point for more complex simulations. In some cases, depending on the degree of accuracy required, linear elastic assumptions may provide satisfactory results. Several published studies of fluid-structure interaction in the cardiovascular system show acceptable results with regards to wall displacements using linear elastic models [12] [2:3] [66]. However, difficulty arises when estimating the elastic modulus. A study by Okamoto et al. [58] examined the mechanical properties of the ascending aorta noting that age and blood pressure created wide variability in elastic modulus. In the Okamoto study, values of approximately $3.5 \mathrm{MPa}$ were observed where other studies cite values between $840 \mathrm{kPa}$ [2.9] to $6.5 \mathrm{MPa}$ [55]. Such variability makes validating results very difficult. 


\begin{tabular}{llll} 
Property & Intima & Media & Adventitia \\
\hline Young's Modulus, E (MPa) & 0.3856 & 1156.928 & 0.3856 \\
Poisson's Ratio, $\nu$ & 0.49 & 0.49 & 0.49 \\
Thickness (mm) & 0.2 & 1.2 & 0.6
\end{tabular}

Table 4.3: Composite model properties [2.9].

Despite this, the stability of linear isotropic properties in a coupled simulation provides an attractive alternative to nonlinear constitutive laws.

\section{Composite Elastic}

Reviewing Fig. 2.5 shows the three fundamental layers found in vascular tissue. Assuming each layer is treated in an elastic isotropic fashion, material properties for each layer have been presented by Gao et al. [29]. This composition should represent the tissue behaviour with greater accuracy over the fully homogeneous model discussed in Section $4.2 \mathrm{~J}$. To evaluate the model stability in the context of a coupled EST analysis, several test simulations were conducted using the properties in Table 4.3. The results of these tests are presented in Section 52.2 .

\subsubsection{Hyperelastic Representations}

A hyperelastic solid is generally defined as a material which can tolerate large strains that are recoverable without any plastic deformation. Common examples include polymers such as rubber, plastic, etc [2]. This is also one of the most common representations of biological materials. Numerous hyperelasticity relations exist, normally derived to represent polymers. However, some models have been developed or modified specifically for use with biomaterials. The most common representation of hyperelastic constitutive relations is through a strain energy formulation. Strain energy can be simply defined as the potential energy stored in a solid due to an elastic deformation. The strain energy can be related to the stress in a solid using Eqn. 4.3 [15],

$$
\sigma_{i j}=\frac{\partial W}{\partial \epsilon_{i j}}
$$

where $W$ is the strain energy function, $\sigma_{i j}$ represents the nine term stress tensor, and $\epsilon_{i j}$ represents the strain tensor [27]. Based on this equation, many strain energy functions can be developed for various materials, with their derivatives giving the direct stress-strain relationships. For convenience, if the material is assumed to be isotropic, the strain energy function $W$ can be expressed in terms of its strain invariants $W=W\left(I_{1}, I_{2}, I_{3}\right)$. Further, the parameter $F_{i j}$ can be defined as the volume-preserving component of the deformation gradient and the parameter $J$ is used to represent the determinant of this deformation component. Building from this, the strain energy function can be defined generally as 
Eqn. $4.4[2]$.

$$
\sigma_{i j}=\frac{\partial W\left(I_{1}, I_{2}, J\right)}{\partial \epsilon_{i j}}
$$

\section{Neo-Hookean Model}

In literature, several strain energy functions have been used to model cardiovascular tissue. One of the simplest is the Neo-Hookean Model. Research by De Hart et al. [2:3] utilized this model successfully in an analysis of the aortic valve. The constitutive relation is represented in Eqn. 4.5

$$
W=\frac{\mu}{2}\left(I_{1}-3\right)+\frac{1}{d}(J-1)^{2}
$$

where $\mu$ is the shear modulus, $I_{1}$ is the first invariant, $J$ is the determinant of the deformation gradient, and $d$ is the material compressibility parameter [2]. This model has the advantage of capturing some of the nonlinearity of biological tissue compared to a perfectly linear elastic model. However, it is generally limited to smaller strains, which may not be adequate for modeling structures like valve leaflets, which experience high deflections and strains. Another main disadvantage in using this model alone is that it is built on isotropic assumptions. As the tissue is known to be highly anisotropic, this is likely to introduce additional error in an analysis. Nonetheless, it is a relatively simple approximation to implement making it advantageous in more complex, coupled analysis.

\section{Mooney-Rivlin}

Another commonly used model is the Mooney-Rivlin relation. Several studies have shown good correlation of this model to cardiac tissue [44] [35] [21] [36] [68][81]. As it is nonlinear in nature, the model is adjusted based on coefficients derived from experimental curve fit data. The relation is available in various orders based on the number of parameters. Popular formulations include 2, 3, 5, and 9 coefficient versions. Studies have shown that the most applicable form for cardiovascular tissue modeling is the five-parameter version [ [2]], which is represented in terms of stress invariants as Eqn. 4.6] [2].

$$
W=c_{1}\left(I_{1}-3\right)+c_{2}\left(I_{2}-3\right)+c_{3}\left(I_{1}-3\right)^{2}+c_{4}\left(I_{1}-3\right)\left(I_{2}-3\right)+c_{5}\left(I_{1}-3\right)^{2}+\frac{1}{d}(J-1)^{2} .
$$

This model has the advantage of an experimental curve fit, theoretically providing a very accurate representation of experimental data. In addition, the hyperelastic function supports higher strain levels making it more versatile for modeling valve leaflets and other large deflection structures. However, as with the Neo-Hookean model, the model is purely isotropic and therefore needs further manipulation or incorporation into an anisotropic relation using different coefficients for each fibre direction to achieve the highest fidelity. 


\subsubsection{Nonlinear Anisotropic Models}

The constitutive relations presented thus far each capture specific elements of the tissue behaviour such as the hyperelastic, nonlinear response, or the anisotropic behaviour, although no model has coupled these into a truly accurate constitutive model. Research by Holzapfel et al. [37] has attempted to address this by creating a simple strain energy framework that incorporates the hyperelasticity of the collagen fibers, linear elastic behaviour of the elastin, and describes each material anisotropically. The first step is to treat each layer of the artery independently, and assign the respective properties based on the specific ratio of collagen to elastin found in each layer. This way each layer of the artery will have a respective strain energy function appropriate to the histologic makeup of that layer. Each model is then built using the principle of superposition, separating the isotropic and anisotropic components into separate terms represented in Eqn. 4.7 [37]

$$
W\left(F, a_{01}, a_{02}\right)=W_{i s o}(F)+W_{\text {aniso }}\left(F, a_{01}, a_{02}\right),
$$

where $W_{i s o}$ represents the isotropic response of the tissue, which is mainly a result of the elastin. $W_{\text {aniso }}$ represents the anisotropic response dominated by collagen which activates at higher strain levels. $F$ is the deformation gradient, and $a_{01}$ and $a_{02}$ are the anisotropic direction vectors. By introducing an appropriate hyperelastic strain energy function for $W_{\text {iso }}$ and $W_{\text {aniso }}$, the model can be tailored to the desired response. For example, a Neo-Hookean model can be implemented to represent the isotropic elastin behaviour at lower strain, while a Mooney-Rivlin or polynomial model can be implemented in anisotropic layers to model the higher strain experienced by the collagen tissue [27]. By applying a version of this model to each artery layer, with material constants tailored to the response of that layer, a very accurate constitutive model can be created. This model is the most comprehensive and versatile in describing the response of cardiovascular tissue however can create substantial complexity when discretizing for a numerical scheme. As there are several layers of nonlinearity including deflection, material properties, contact, etc., orchestrating reliable convergence can be very challenging [37]. Also, as with any nonlinear model, obtaining accurate experimental parameters from biological tissue is not trivial.

\subsubsection{FEM Solution Methodology}

After a comprehensive evaluation of the constitutive models presented in Section 4.2.1, the linear elastic and composite models were chosen for implementation into the coupled ESTl model. This decision was based on the practicality of obtaining converged solutions in a coupled fluid-structure simulation. Although theoretically, a nonlinear model provides the most accurate representation of the true artery wall behaviour, locating suitable data for the aortic arch tissue prevented the integration of such a model into the final coupled analysis.

According to the theory presented, the most accurate linear representation presented is the composite 


\begin{tabular}{ll} 
Property & Value \\
\hline Young's Modulus, E & $3.0 \mathrm{MPa}$ \\
Poisson's Ratio, $\nu$ & 0.49 \\
Thickness [30] & $2 \mathrm{~mm}$
\end{tabular}

Table 4.4: Linear isotropic material parameters.

model, which endeavours to incorporate the behaviour of each of the arterial layers. Although successful static solutions were generated, in the coupled EST simulation, extensive convergence problems were encountered preventing any successful results. Therefore the linear elastic model was ultimately chosen based on the most accurate parameters provided in literature.

All finite element analysis was conducted using the commercial code MSC. Marc (MSC Software Corp.). All elements were defined as three-dimensional shell elements with a thickness of $2 \mathrm{~mm}$. The material parameters used are defined in Table 4.4, adapted from Beller et al. [1.3]. The model was constrained in all degrees of freedom at the aortic inlet and all outlets. Static simulations were conducted using a ramped load case with applied force distributed over 10 sub steps. In the coupled $\mathbb{E S T l}$ case, a dynamic transient load case was used with global time parameters matching those of the unsteady CFD analysis. All solutions were conducted using a multifrontal sparse solver which provides the most efficient solution of a well conditioned linear system on a multi-processor computer [3].

\subsection{Fluid-Structure Integration}

The software package Mesh-based Parallel Code Coupling Interface (MpCCI) (Fraunhofer SCAI, Germany) was chosen as the intermediate code responsible for handling the fluid-structure coupling. The software is structured in two main components, a code adaptor that is written to interface with the specific solver, and a server which coordinates the exchange of variables between the solvers, in this case, MSC.Marc and FLUENT. MpCCI uses a weak coupling approach to solving coupled systems. This segregates the governing equations into separate sub-problems, which can be solved by independent solvers (Fig. 4.4). In this configuration, the flow field including gauge pressure at each node is calculated first. This data is then sent to the solid solver, MSC. Marc, which then computes the corresponding nodal displacements. These displacements are subsequently transferred back to the CFD solver, FLUENT, which perturbs the mesh and recomputes the solution. This interaction constitutes one time step. This is in contrast to a strong coupling system, where all equations are solved simultaneously. Although strongly coupled systems tend to create more precise solutions by reducing the interpolation between domain boundaries, in this situation weak coupling is more flexible by reducing the need for further software development [6]. 
code A

code B

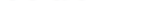

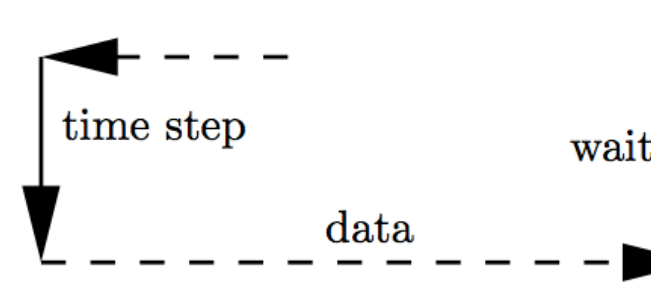

wait for data

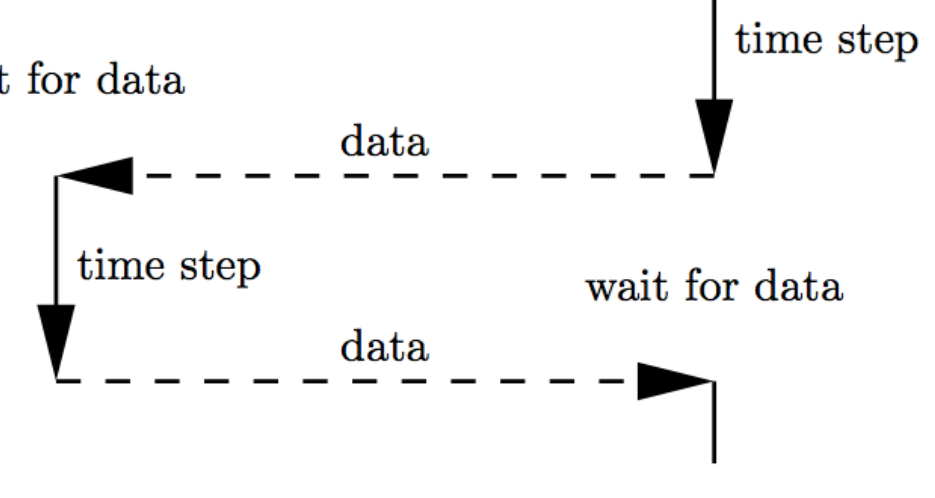

Figure 4.4: Staggered weak coupling algorithm used by MpCCI [6].

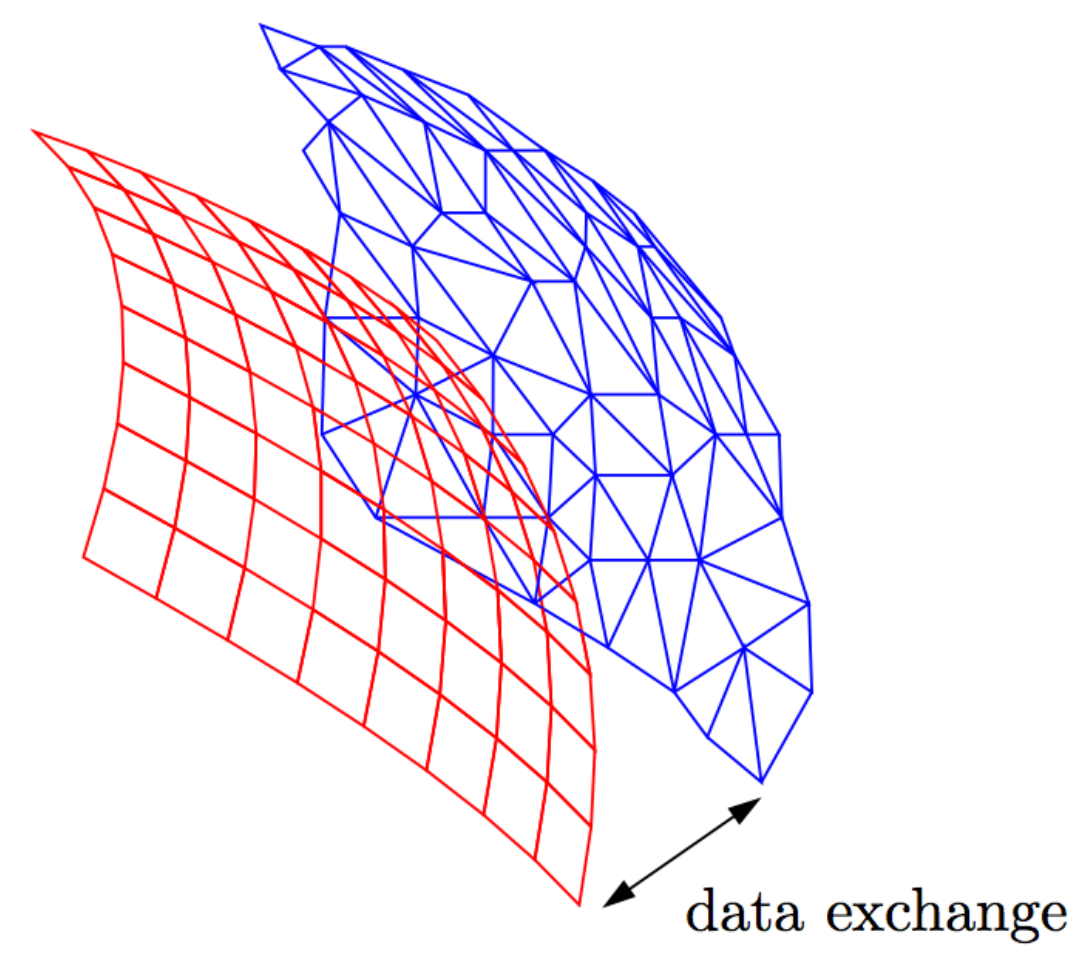

Figure 4.5: Nonmatching FEM-CFD meshes [6]. 


\subsubsection{Boundary Data Exchange}

To simplify the data exchange, MpCCl also allows independent or non-matching grids to be used in each solver (Fig. 4.5). This simplifies the meshing process substantially and allows optimal element choice for the fluid and solid regimes. As a result of the non-matching grids, MpCCI cannot directly transfer nodal forces and displacements between meshes. To bridge the two meshes together, a two stage approach is employed where similar nodes on each grid are associated together and the resulting forces are interpolated between these associated nodes. To determine the associated nodes on the fluid-solid interface, a neighbourhood search is conducted based on a Kd-tree approach where a bounding box is created around each mesh node based on preset criteria [6].

Once the associated nodes between the fluid and solid mesh are defined, a shape function algorithm is used to interpolate the coupled pressure and nodal position from one mesh to the next. The requirement for this is that the element order on each grid be consistent (linear or quadratic).

For this $\mathbb{E S T l}$ simulation, nodal position and gauge pressure are exchanged between the two solvers. The solution is first initialized in FLUENT, and the resulting gauge pressures are transferred to MSC.Marc. This solver then computes the resulting stress and strain for post-processing, and transfers the resulting nodal displacements back to MpCCI, which deforms the FLUENT mesh via the moving mesh user-defined function. The fluid solution is then recomputed using the deformed mesh, and the cycle continues over the stipulated simulation duration.

Solution specific information such as artery wall stress and strain, or fluid velocity and pressure are then retrieved and post-processed using the respective solver environments or a unified post-processing program such as TecPlot. 


\section{Chapter 5}

\section{Results}

\subsection{Mesh Independence Study}

Among the first tests conducted was a grid independence study. This involved performing several iterations of the solution using a progressively finer mesh until it could be shown that the solution was independent of the mesh size.

For the fluid mesh, three steady-state simulations were conducted with mesh densities ranging from 55,248 to $1.4 \mathrm{M}$ elements. Mass flow rate through the left common carotid artery was used to represent the solution on each mesh. The results of this independence study are displayed in Fig. 5.1 which plots the difference in solution for each mesh relative to the finest mesh studied. This effectively shows a reduction in error in each solution, proportional to the mesh size. The absolute difference in the calculated mass flow rate of the coarse mesh relative to the finest is $10^{-} 3$. Therefore, for computational efficiency, a mesh of 118,590 elements was chosen for subsequent unsteady [CWD and EST solutions.

\subsection{Steady State Results}

\subsubsection{Steady CFD}

Although the nature of the problem centres on highly pulsatile flow, steady state simulation still provides an important reference for initializing the more advanced unsteady simulation. Also, comparison between steady and unsteady cases can provide important insight into the implications of pulsatile flow. 


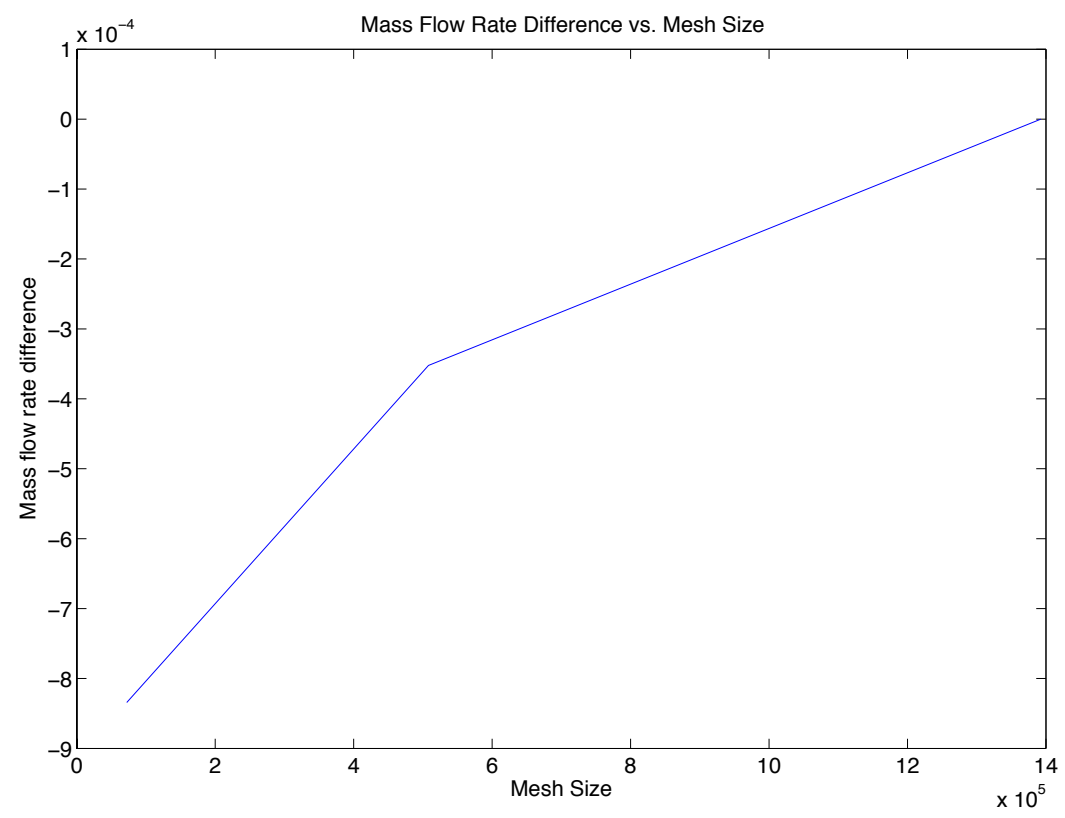

Figure 5.1: CFD mesh independence study showing LCC mass flow rate verses mesh density.

\section{Simulation Conditions}

The steady state simulations were carried out using a constant pressure defined at each boundary. The inlet pressure was set to a constant $16 \mathrm{kPa}$, matching normal systolic blood pressure. Each outlet was assigned a pressure boundary set at $10665 \mathrm{~Pa}$, coinciding with diastolic blood pressure. Remaining

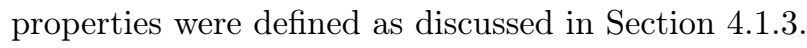

\section{Results}

The results of the steady state simulations (Fig. 5 ) show that continuity is satisfied across all boundaries, and the mass flow distribution is consistent with anatomy, with the larger arteries experiencing higher mass flow, proportional to their diameter.

Fig. 5.3 illustrates velocity streamlines obtained from this steady simulation. It is noted that even in the steady case, representing the peak systolic pressure state, that no appreciable turbulence was found. Even as the flow splits off into the ascending arteries, it remains primarily laminar.

Fig. 5.4 shows contours of static pressure on the arterial wall. As expected, higher pressures are experienced closer to the aortic valve and drop toward the descending aorta accompanying the increase in flow velocity. Fig. 5.4 also shows the relationship between wall pressure and geometric contours. This draws attention to the importance of accurately modelled geometry if conclusions regarding pressure 


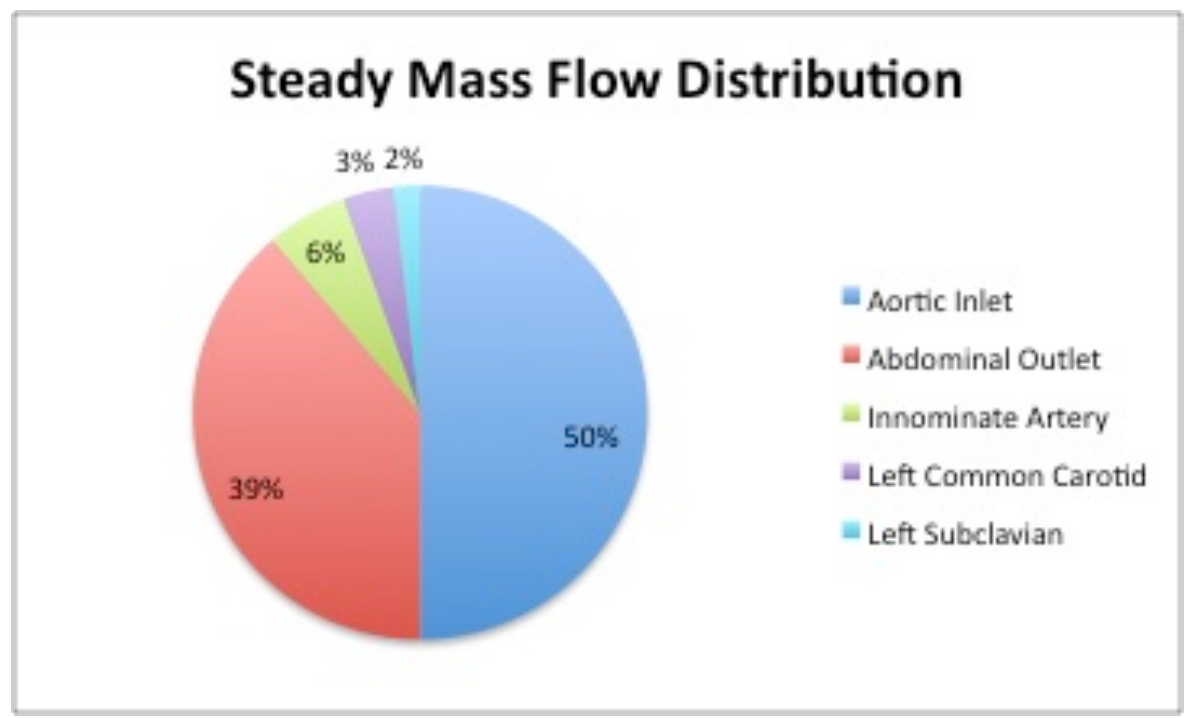

Figure 5.2: Steady mass flow distribution across boundaries.

distribution are to be made.

\subsubsection{Static FEM Results}

Preliminary analysis was conducted on two constitutive models to determine both the feasibility of integration into an $\mathbb{E S T l}$ analysis as well as the expected accuracy of the solution. The models chosen include a homogeneous linear elastic model, and three-layered composite linear elastic model. With regards to feasibility, the primary goals were to determine which models would provide adequate convergence and which have enough published experimental data to make an accurate nonlinear analysis possible.

\section{Simulation Conditions}

All models were tested using identical meshes, and in the anticipated ESTl configuration. To simulate systolic pressure, a $16 \mathrm{kPa}$ face load was applied to all interior elements. The model was fixed in all degrees-of-freedom at each inlet and outlet. This is a reasonable assumption as the aortic arch does not translate significantly through the cardiac cycle. Type 75, thick shell elements with an assigned thickness of $2 \mathrm{~mm}$ were used. Remaining parameters were defined as per Section 4.2.4. Wall displacement and von Mises stress were chosen as the comparison parameters for each model. 


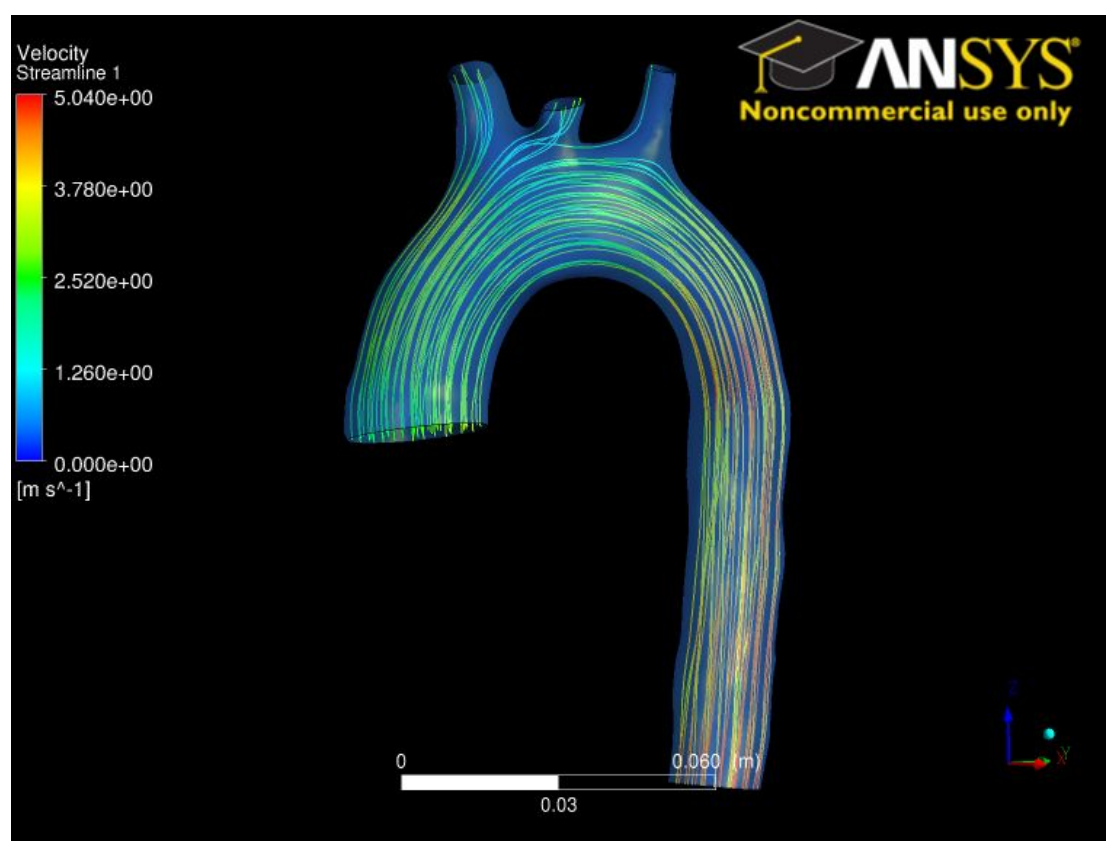

Figure 5.3: Steady velocity streamlines.

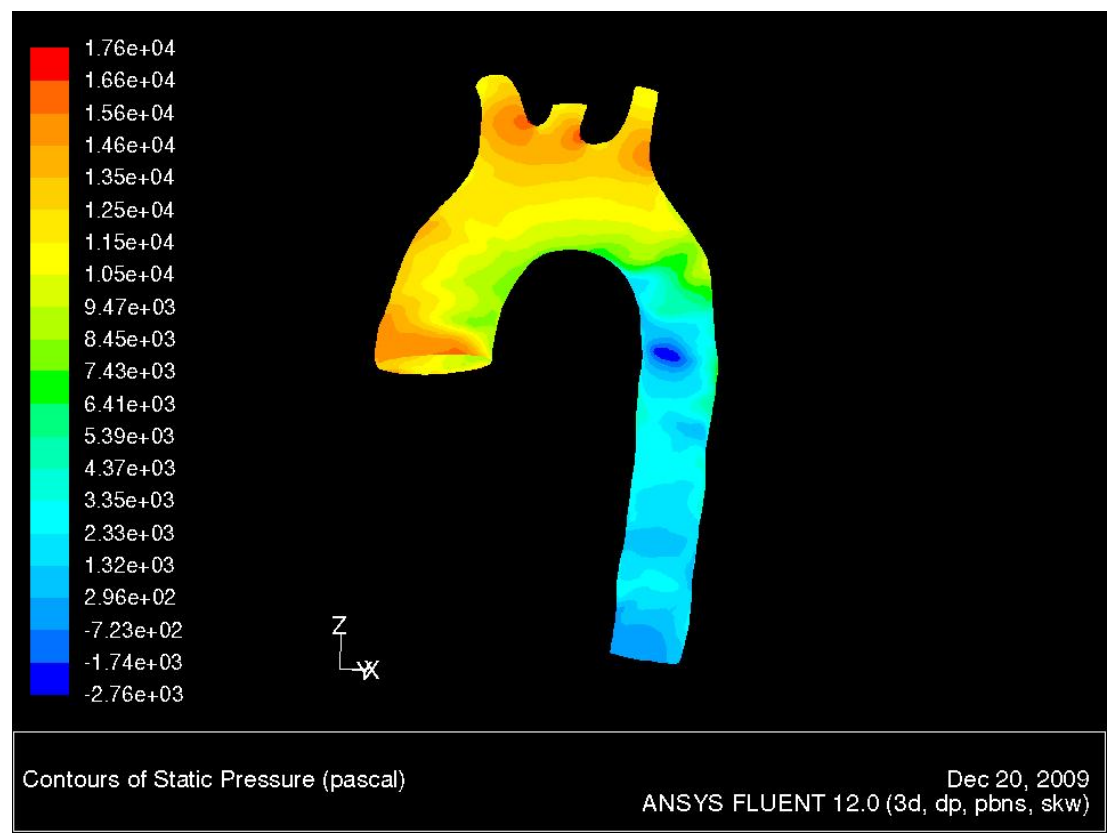

Figure 5.4: Contours of static pressure (steady state). 


\begin{tabular}{lll} 
Parameter & Maximum & Minimum \\
\hline Displacement & $2.27 \mathrm{~mm}$ & - \\
von Mises Stress & $272 \mathrm{kPa}$ & $12.8 \mathrm{kPa}$ \\
\hline
\end{tabular}

Table 5.1: Summary of static linear elastic results

\begin{tabular}{lll} 
Parameter & Maximum & Minimum \\
\hline Displacement & $3.28 \mathrm{~mm}$ & - \\
Stress & $264 \mathrm{kPa}$ & $13.8 \mathrm{kPa}$ \\
\hline
\end{tabular}

Table 5.2: Summary of static composite model results.

\section{Linear Elastic Model}

The linear elastic model used a constant Young's modulus of $4 \mathrm{MPa}$ and a nearly incompressible Poisson ratio of 0.49. Table 5 . 1 outlines the minimum and maximum results for tissue displacement, and von Mises equivalent stress. Fig. 5.5(a) shows the contours of displacement in the static case. The peak displacements occur on the sides of the arch below the left common carotid artery. This is likely due to the cross sectional shape of the artery in this region which is more rectangular and therefore more prone to deflection than a similar circular cross section.

The von Mises equivalent stress distribution is shown in Fig. 5.6(a). The contours of stress show that the distribution, like pressure, is highly dependant on model geometry. As the wall contours are likely to vary substantially between models, specific comparison of stress distributions would be difficult. Peak stresses are found in the web regions between the branching ascending arteries. However, it is important to note that the proximity of the fixed boundary conditions to these regions may strongly affect the magnitude and distribution in these regions.

\section{Composite Model}

A composite model based on the constitutive representation developed in Section 4.2.1 was also evaluated under the same conditions outlined in Section 5.2.2. Fig. 5.5 compares the displacements of both the linear elastic and composite model. The most apparent difference is the substantial drop in peak displacement. Experimental values indicate expansion in the aortic arch to be on the order of 2 to $3 \mathrm{~mm}$. The approximate expansion in diameter in the linear elastic model is $4.54 \mathrm{~mm}$ and $3.28 \mathrm{~mm}$ in the composite model (Table. 52). With experimentally measured values ranging between 2 and 3mm [5] , it is clear the composite based model, approximating the individual contribution of each artery layer is substantially more accurate than the homogeneous linear elastic model. Although the distribution of displacement magnitude is similar between each model, the composite simulation shows slightly higher displacement gradients in the descending section.

Comparing von Mises stress represented in Fig. 5.6, similar distribution patterns are noted in both models. Peak stresses are also on the same order of magnitude ranging between 257 and $272 \mathrm{kPa}$. 


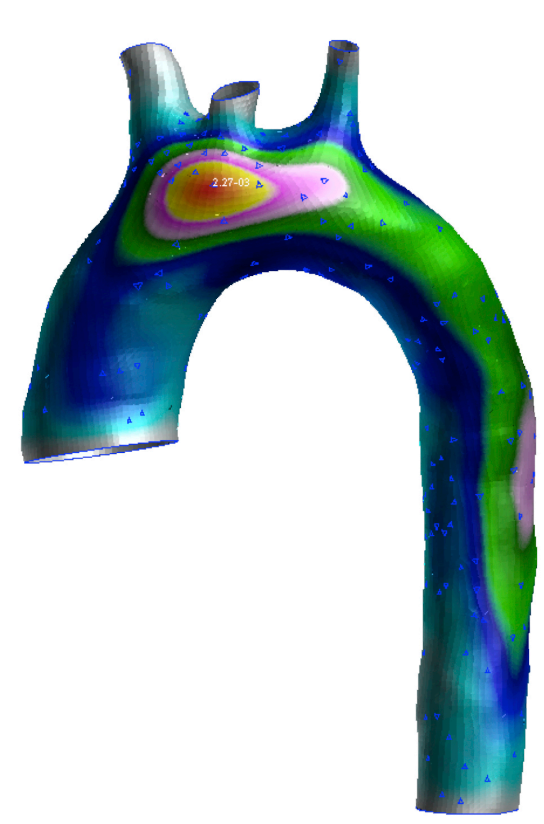

(a) Static displacement of homogeneous linear elastic model under $16 \mathrm{kPa}$ load.

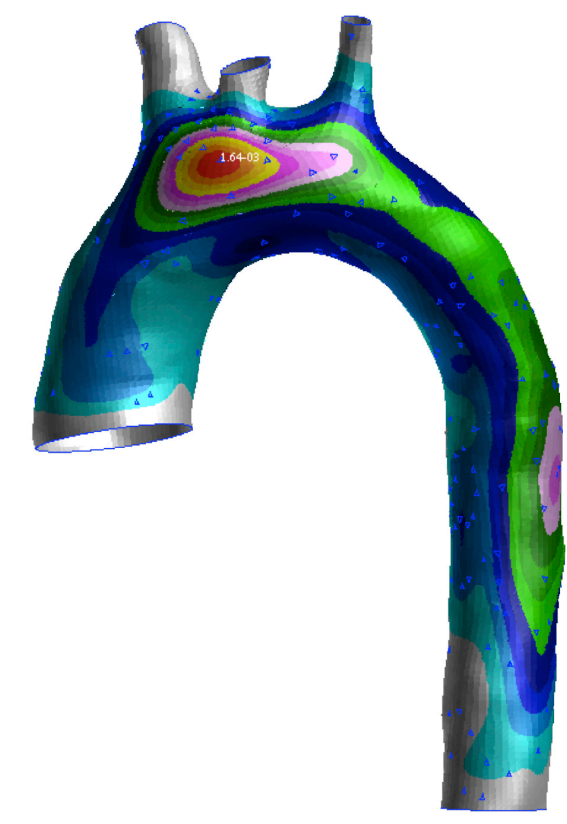

(b) Static displacement of composite linear elastic model under $16 \mathrm{kPa}$ load.

Figure 5.5: Comparison of wall displacement between homogeneous and composite linear elastic models.

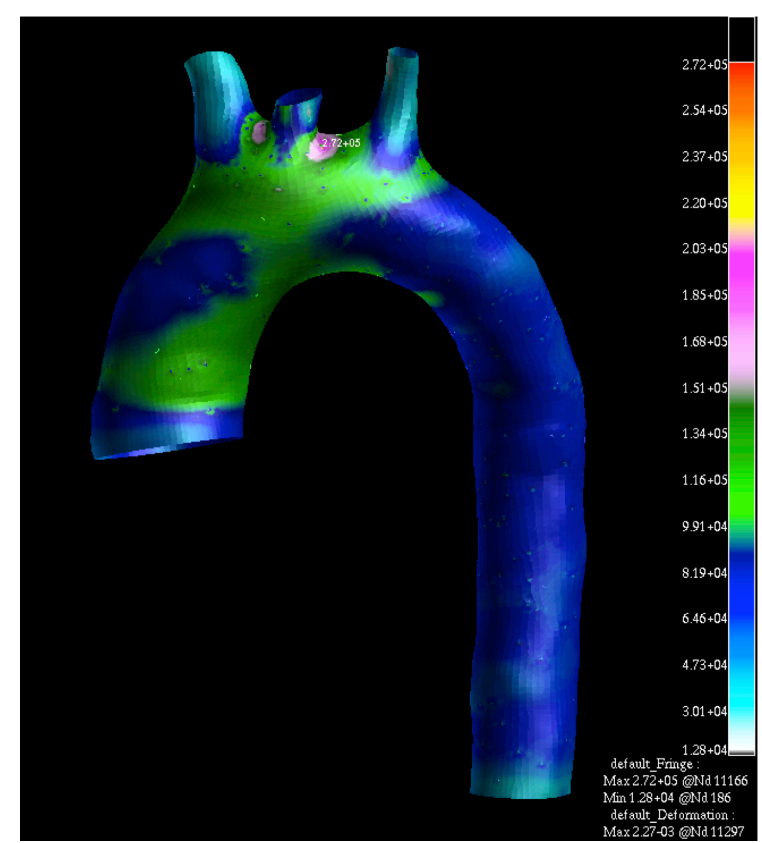

(a) von Mises stress distribution of homogeneous linear elastic model under $16 \mathrm{kPa}$ load.

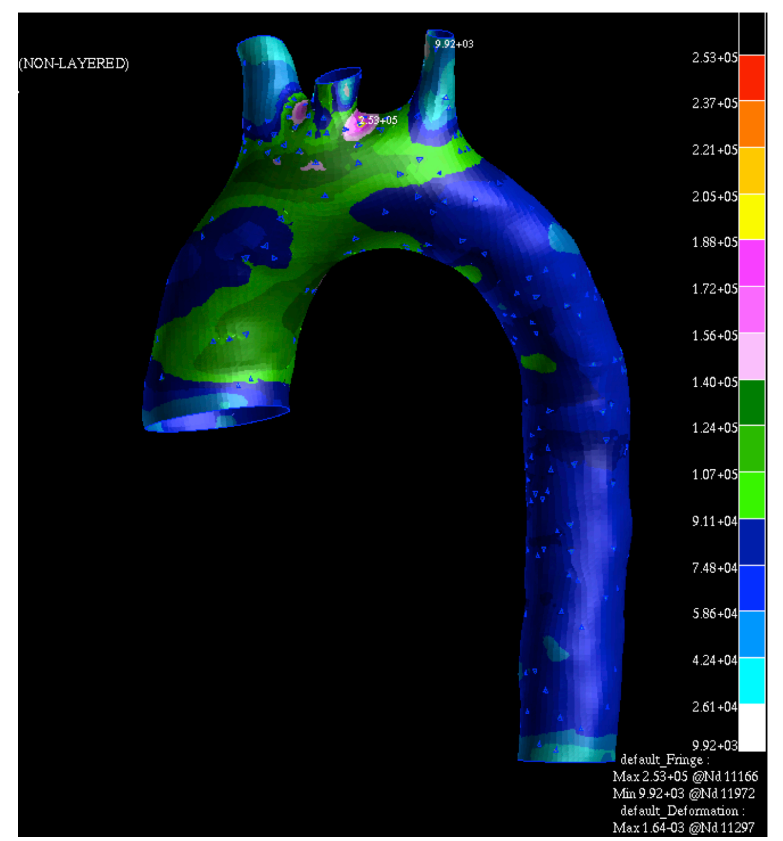

(b) von Mises stress distribution of composite linear elastic model under $16 \mathrm{kPa}$ load.

Figure 5.6: Comparison of von Mises stress between linear and composite elastic models. 


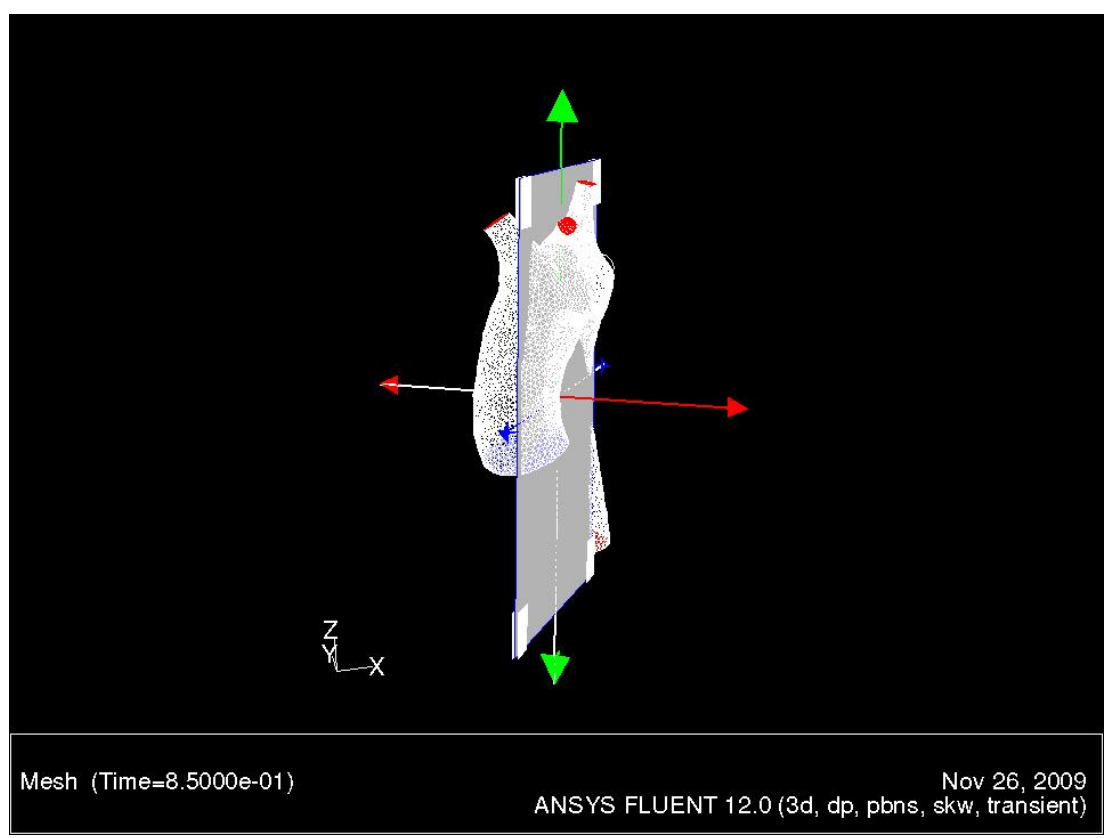

Figure 5.7: Diagram showing CFD arch bisection plane position.

It is clear that the composite model more accurately represents experimentally measured behaviour at $1 \mathrm{G}_{\mathrm{z}}$ and would be the ideal linear elastic model to include in the final $\mathbb{E} S \mathrm{Tl}$ simulation. However, in tests with a fully coupled model, substantial difficulty obtaining solution convergence was encountered, preventing the inclusion of this constitutive relation in the final model.

\subsection{Unsteady Results}

\subsubsection{Overview}

Initial unsteady simulations were conducted using a time step size of $0.1 \mathrm{~s}$ to a total of 200 steps. This allowed the visualization of 4 cardiac cycles. Each time step was allowed to compute until a minimum $10^{-5}$ residual error was achieved.

\subsubsection{Velocity and Mass Flow}

Fig. 5.8 shows the velocity vectors across a lateral plane bisecting the arch (Fig. 5.7 ) near peak flow at normal earth gravity $\left(9.81 \mathrm{~m} / \mathrm{s}^{2}\right)$. Across the mid-plane the average velocity ranged between $0.5 \mathrm{~m} / \mathrm{s}$ to $1.22 \mathrm{~m} / \mathrm{s}$ with localized regions peaking near $1.76 \mathrm{~m} / \mathrm{s}$. This corresponds well to experimental data obtained in the ascending aorta (Table 5.3 ). 


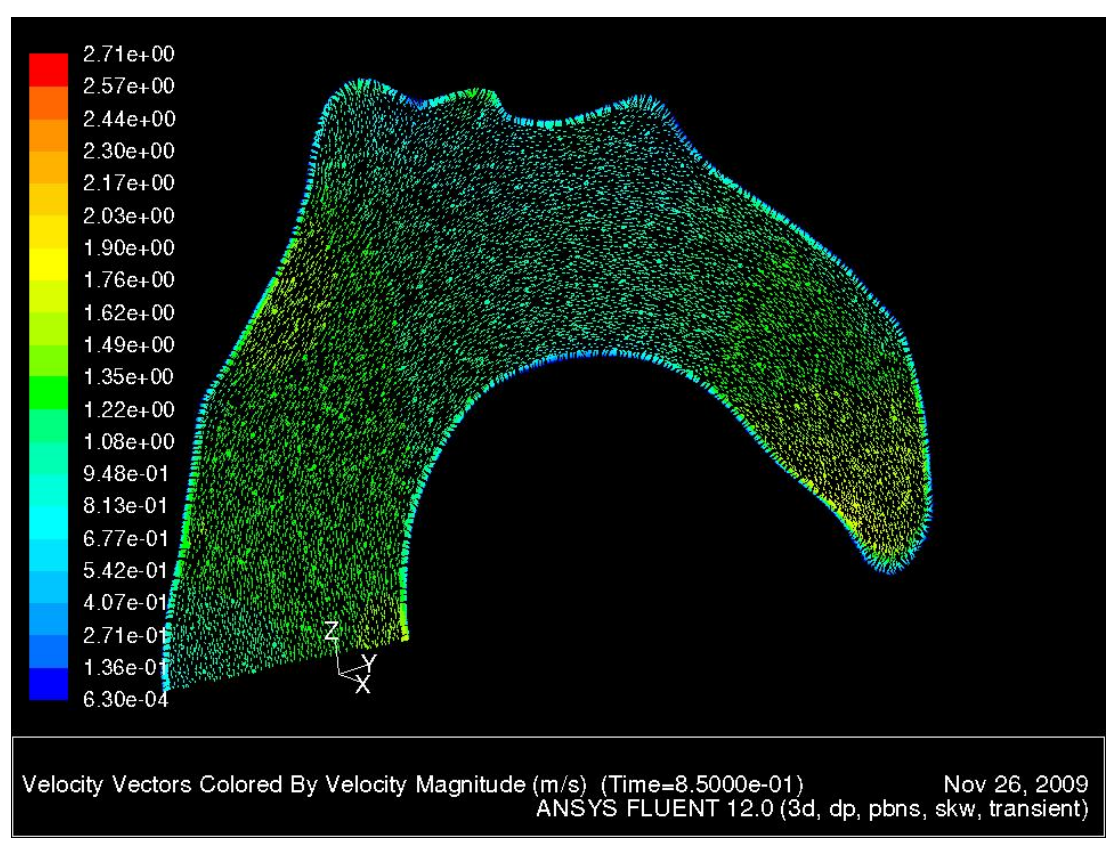

Figure 5.8: Velocity vectors across the mid-plane of the arch.

\begin{tabular}{l|l} 
Modality & Average Peak Velocity $(\mathrm{m} / \mathrm{s})$ \\
\hline Catheter-Tip Hot-film Anemometry [7] $]$ & 1.27 \\
Phase Encoded MR] [48] & 1.3 \\
Presented Unsteady CFD Analysis & $\mathbf{1 . 2 2}$
\end{tabular}

Table 5.3: Comparison of peak aortic arch velocities by various modalities.

Table 5.3 illustrates a comparison of the numerical results for peak flow velocity with results measured using various experimental techniques. It is important to identify that Table 5.3 shows the agreement of the CFD study with averaged values obtained from other modalities. As all studies were not performed on the same patient or using the same geometry, conclusions regarding the accuracy of one modality versus another should not be drawn, only the general agreement of results obtained from various methods.

With the standard gravity control case showing strong correspondence with published results, gravitational load was added to the model in $1+\mathrm{Gz}$ increments through the addition of a $\mathrm{Z}$ momentum source term in the governing equations. The first noted result was the increase in velocity through the descending aorta (Fig. 5.

Corresponding with the rise in aortic velocity, substantial changes in boundary mass flow rates were noted. Through the descending outlet, a linear increase of approximately $15 \%$ per G was calculated. Accompanying the increase in abdominal flow was a nearly linear reduction of outward mass flow from the ascending arterial branches (Fig. $5 . \mathrm{C}$ ). Since the left ventricle (feeding the inlet) has a finite volume of fluid, any increase in flow through the descending region must be drawn from ascending branches. At 


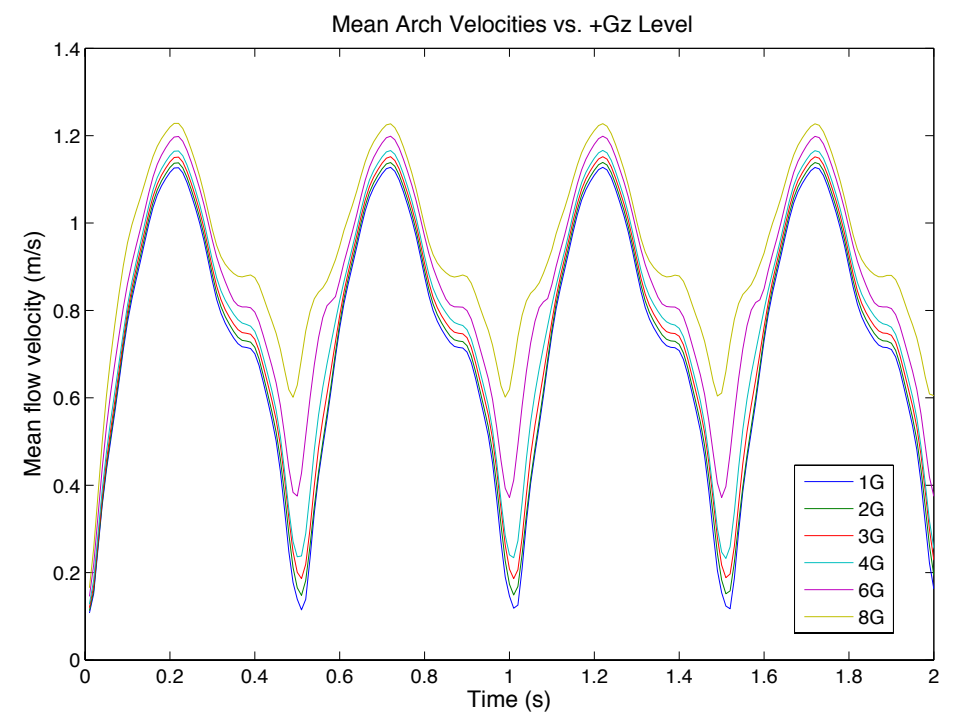

Figure 5.9: Effect of inertial load on average velocity at the top section of the arch.

approximately $8+\mathrm{Gz}$, all outward flow to the left common carotid artery ceased (Fig. important physiologic implications, indicating complete loss of upper body pertusion.

\subsubsection{Pressure}

Fig. 5.5 represents the contours of static pressure during the systolic phase of the unsteady analysis. Comparing with those in the static investigation (Fig. 5.4), the contours of the pulsatile flow are lower in magnitude across the entirety of the wall and particularly in the ascending branches. This is due to the transient nature of the pressure pulse which prevents the pressure from reaching its equivalent steady value.

The effect of inertial load is illustrated in Fig. 5. 13. As inertial load is applied, the hydrostatic pressure opposes the systolic pressure generated by the contraction of the left ventricle thereby reducing the overall arch pressure. Each increase in $\mathrm{G}_{\mathrm{z}}$ creates a corresponding decrease in pressure of approximately $5 \mathrm{mmHg}$.

\subsubsection{Turbulence and Flow Behaviour}

In Fig. 5.14, a plot of $y+$ values across the wall surface shows a peak $y+$ value of 2.06, although most regions are well under 1 . This verifies the applicability of the k- $\omega$ model used which desires an ideal $y+$ below 2 [回]. 


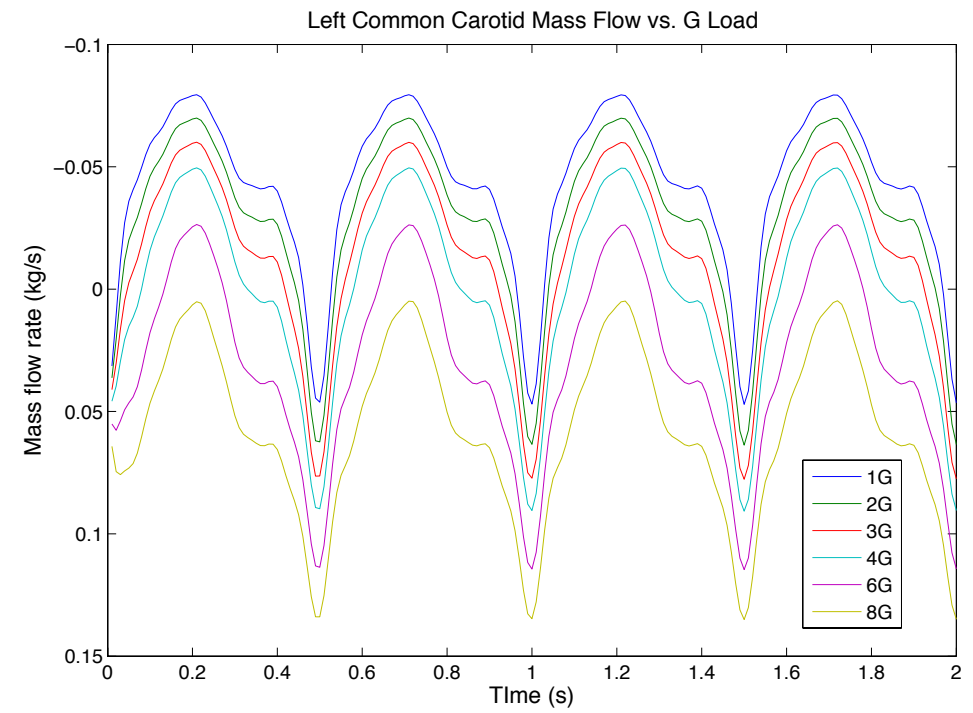

Figure 5.10: Effect of inertial load on unsteady mass flow through the left common carotid artery.

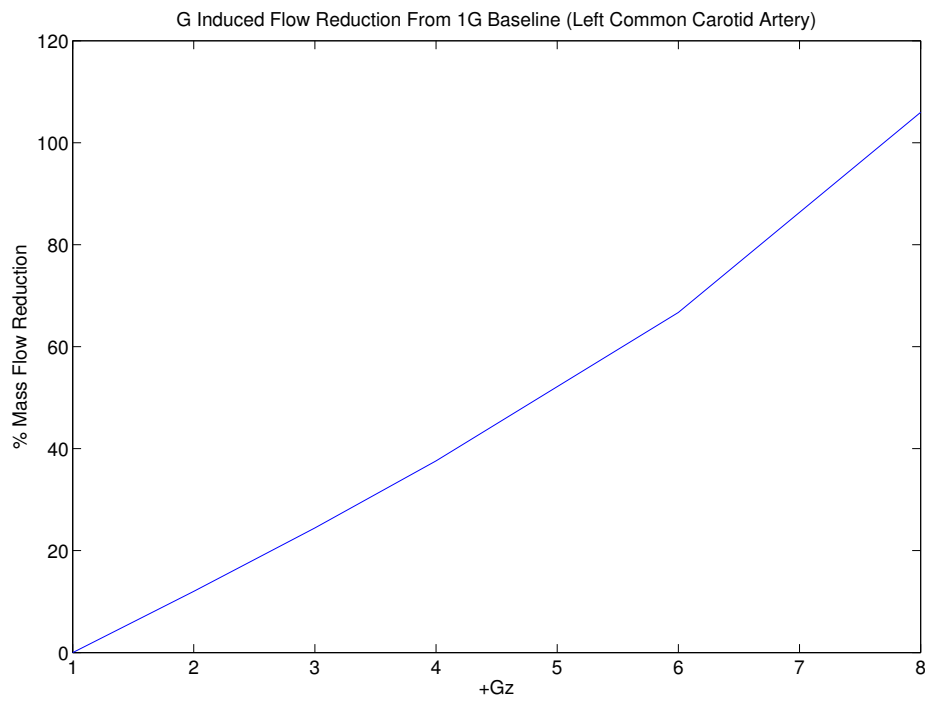

Figure 5.11: Reduction in mass flow rate through the left common carotid artery with increasing inertial load compared with a $1 \mathrm{G}$ baseline. 

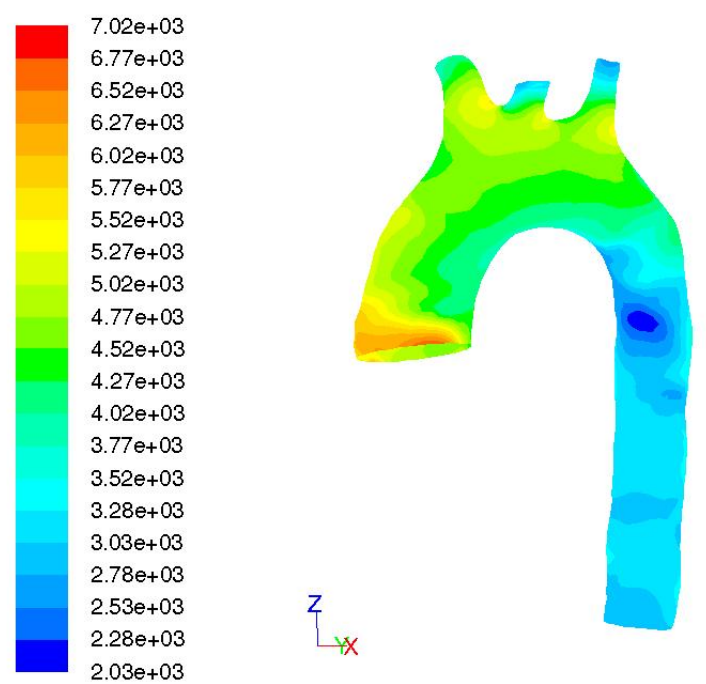

MNYS

Contours of Static Pressure (pascal) $\begin{aligned} & \text { (Time }=7.0000 \mathrm{e}-01) \\ & \text { ANSYS FLUENT } 12.1 \text { (3d, dp, pbns, dynamesh, skw, transient) }\end{aligned}$

Figure 5.12: Pressure contours at systole under standard gravity conditions.

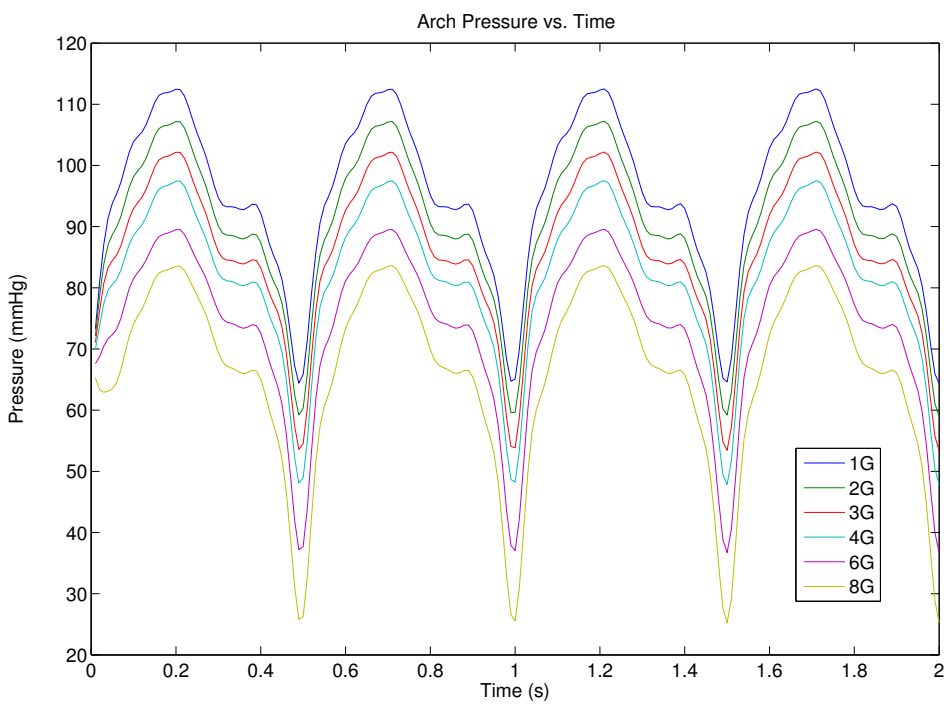

Figure 5.13: Variation in arch pressure under gravitational load. 

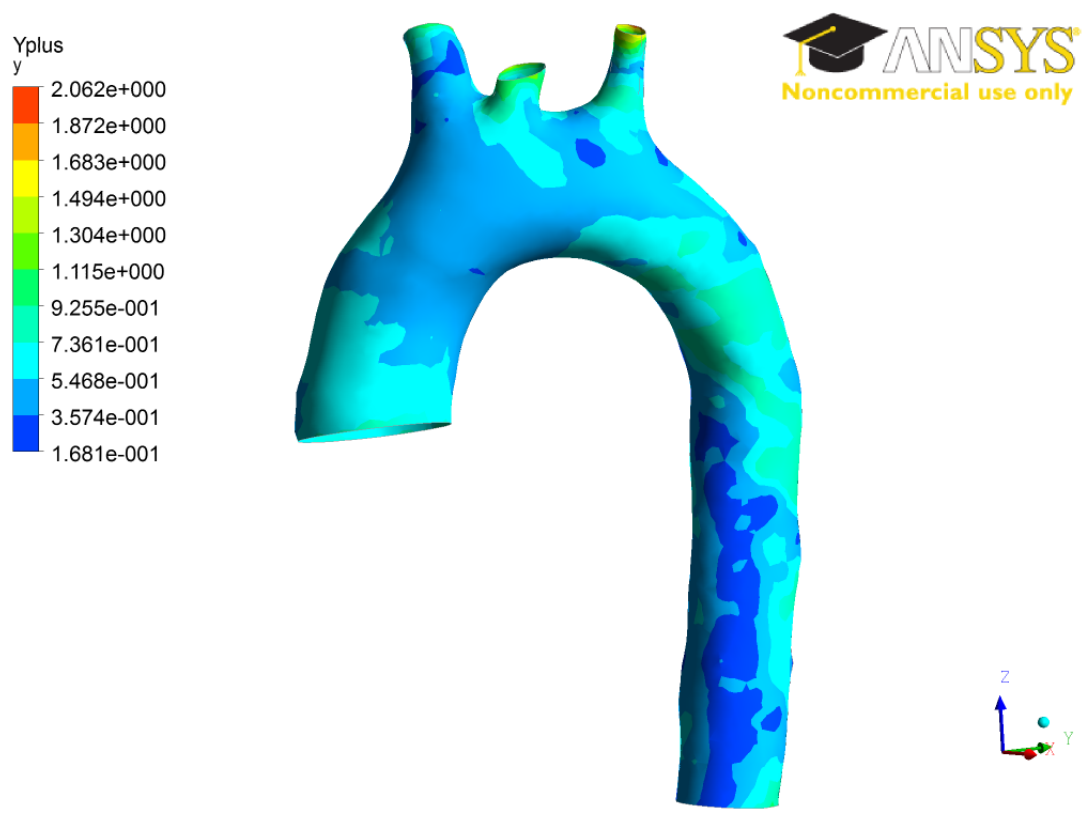

Figure 5.14: Systolic $y+$ contours during an unsteady standard gravity simulation.

When examining the behaviour of the flow throughout systole under standard gravity conditions, many complex structures emerge. The most prevalent is the strong right handed helix which develops throughout the arch, intensifying through the descending aorta. Such flow patterns have been studied both in vivo and in vitro through the use of phase-contrast पMR] [4.5] and colour Doppler ultrasound [26]. Both studies as well as numerous others confirmed the existence of these structures throughout the aorta. Generally the secondary flow is attributed to a skewed velocity profile that forms, accelerating fluid near the outer bend of the arch faster than the inner regions [45]. Once systole has ended, the inner regions decelerate faster creating retrograde flow.

The application of inertial load tended to amplify the flow structures noted in the $1 \mathrm{G}$ case. As the applied load surpassed $6+\mathrm{Gz}$, secondary flow recirculation began to emerge in the ascending portion of the arch. This is likely caused by an amplification of the skewed velocity profile noted in the ascending region. It is plausible that enough inertial load near the inner wall could cause localized flow reversal resulting in an inner wall recirculation region. This behaviour is seen through streamline visualization in Fig. 5.15(b).

In addition to the of creation of localized secondary flow behaviour, the increased gravitational load also acted to strengthen the helical flow behaviour noted in the standard gravity case, particularly in the descending aorta. This is an expected finding, corresponding to the increase in flow velocity through the abdominal outlet. This strengthening effect could have interesting implications on the overall cardiovascular health of pilots, or others exposed to $\mathrm{G}$ forces. Research by Morbiducci et al. [52] examined the link between helix strength in arteries to the development of atherosclerotic plaque formation. Their 


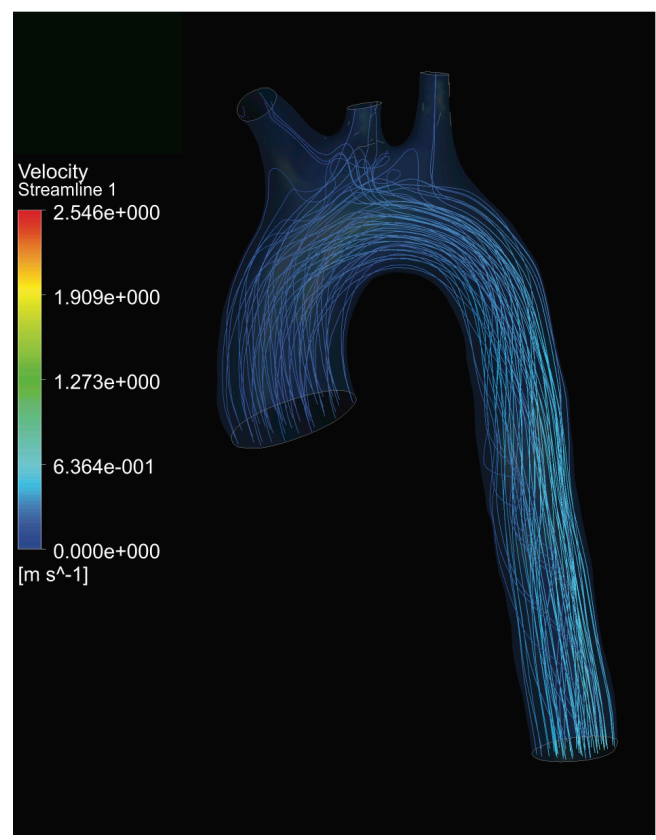

(a) During diastole under standard gravity $\left(1 G_{z}\right)$.

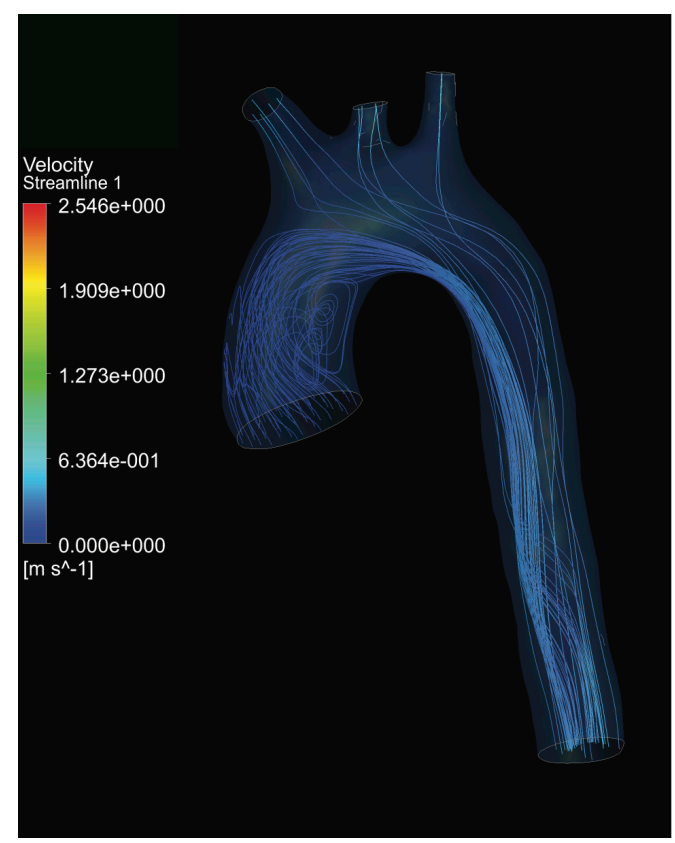

(c) At beginning of systole (ejection) under standard gravity $\left(1 G_{z}\right)$.

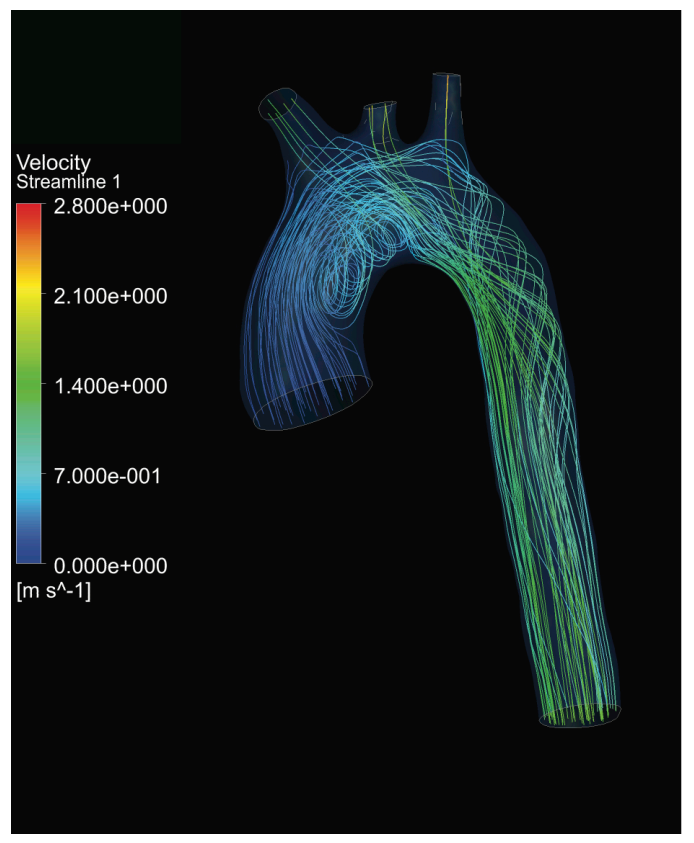

(b) During diastole under 6 times standard gravity showing substantial increase in abdominal aortic flow and recirculation regions in the ascending segment.

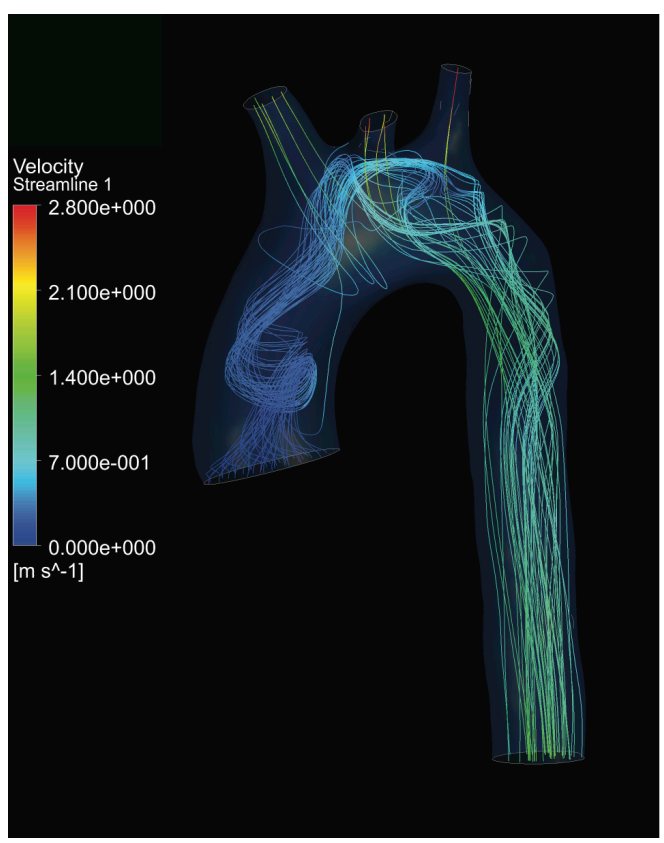

(d) At the beginning of systole (ejection) under 6 times standard gravity. The development of helical flow can be seen clearly in both the ascending aorta.

Figure 5.15: Comparison of velocity streamlines in the aortic arch for $1 \mathrm{G}_{\mathrm{z}}$ and $6 \mathrm{G}_{\mathrm{z}}$. 
research noted that stronger helical flows may reduce the risk for atherogenesis and potentially reduce the risk of cardiovascular disease. This is thought to be a result of the oscillatory component of the wall shear stress, acting to inhibit the formation of plaque [52][62][174].

A by-product of the helical secondary flow is an affect on turbulence. Published data by Kilner et al. [45] indicates that the formation of a helix in the ascending region may minimize separation and subsequently reduce turbulence. Insufficient data and model fidelity prevents verifying these turbulence findings in this numerical study. However, these results provide interesting pathways for future research.

\subsection{Comparison of Unsteady vs. Steady Results}

Comparing the results between the steady and unsteady analysis illustrates a substantial variance in flow properties and behaviour. For example, peak flow velocity in the ascending region was over predicted by the steady state model by a factor of 4 . When using a steady solution for unsteady initialization purposes, the discrepancies were often large enough to generate convergence problems at the beginning of the solution. It was found that initializing using a constant pressure slightly lower than that of the diastolic value provided the most effective simulation start. The steady simulation by its very nature, does not capture important secondary flow behaviour and turbulence. This secondary behaviour, which is modified by inertial load, carries important physiological implications. It is therefore concluded that steady simulations offer limited value in cardiovascular analysis and that data obtained from such simulations should be interpreted cautiously.

\subsection{Coupled Fluid-Structure Results}

There is no profound variation between the unsteady CFD analysis alone and the incorporation of the structural regime. This is expected due to the relatively small $(2-4 \mathrm{~mm})$ deflection of the wall and the time-invariant nature of the outflow boundary conditions. The main benefit of the fully coupled analysis is in the determination of the transient tissue response. Fig. 5.16 compares the deformed tissue wall at systole under $1 \mathrm{G}_{\mathrm{z}}$ and $6 \mathrm{G}_{\mathrm{z}}$. The increase in hydrostatic load tended to damp the deformation at the top of the arch by approximately $0.5 \mathrm{~mm}$. This was accompanied by a slightly larger displacement through the descending region of the aorta, likely due to the increase in mass flow rate under the higher load. The maximum deformation at systole in the $1 \mathrm{G}_{\mathrm{z}}$ case was approximately $4.5 \mathrm{~mm}$ which is slightly higher than the published values of $2-3 \mathrm{~mm}$ in the ascending arch [57]. This is attributed to the simplifications introduced by the linear elastic model as discussed in Section 5.22 . Fig. 5.17 shows the von Mises stress distribution in the artery wall under standard conditions and $6+\mathrm{Gz}$. Although the overall distributions are similar, the inertial load acted to increase stress in regions of the ascending and descending aorta. The peak stress concentrations were slightly reduced due to the hydrostatic load acting in opposition 


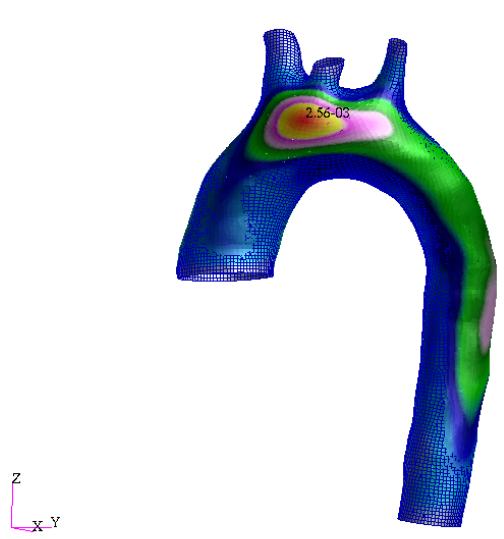

(a) Systolic displacement under $1+\mathrm{Gz}$ load.
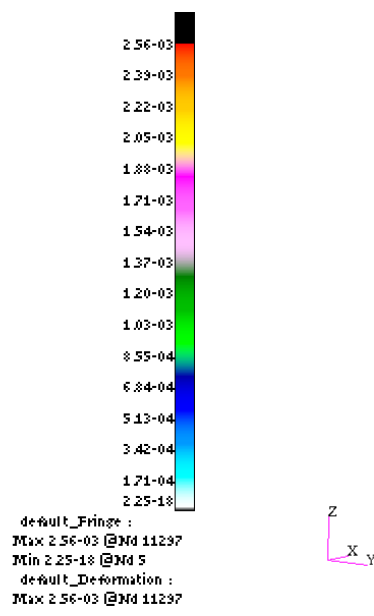

(b) Systolic displacement under $6+\mathrm{Gz}$ load.

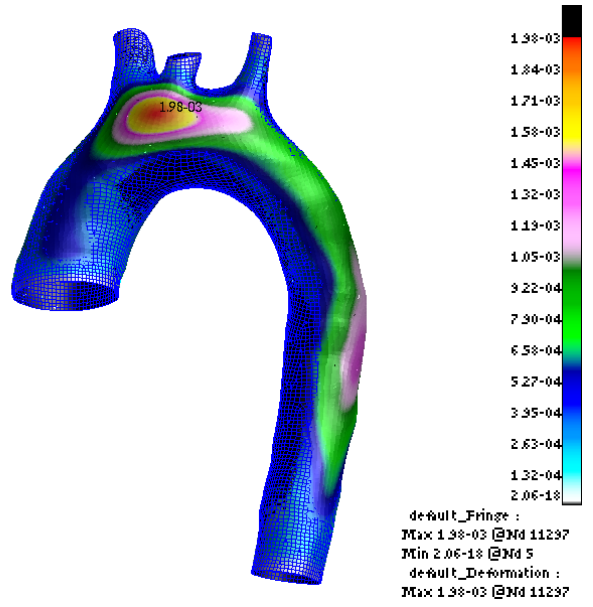

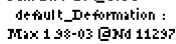

Figure 5.16: Comparison of systolic displacement under inertial load.

to the systolic inflation pressure. Although the results appear intuitive and reasonable, the absence of similar experimental results makes quantitative validation impossible at this time.

\subsection{Computational Considerations}

The scale and complexity of this coupled analysis necessitated the extensive use of parallel computing. Conducting such an analysis in serial would result in unreasonably long solution times. Although both regimes of the problem are tightly coupled, results indicate the problem scales well in a parallel environment. Due to the large mesh size, the CFD side of the problem was the most computationally expensive.

The majority of the results presented were computed with the use of an $8 \mathrm{CPU}$ workstation computer, however some unsteady CFD simulations were computed using up to 40 CPUs on Ryerson University's jvlNet cluster. To specifically evaluate the scalability of the CFD simulation, a test was conducted with a steady state simulation on a $2.1 \mathrm{M}$ element variant of the presented CEDD mesh. The test was run on the SHARCNET Saw supercomputer to a maximum of 64 CPUs. The simulation was allowed to run for 500 iterations and resulting performance values were measured. The resulting performance data is presented in Table 5.4 and Fig. 5.8 . Fig. 5.5 shows that the problem scales remarkably well. 

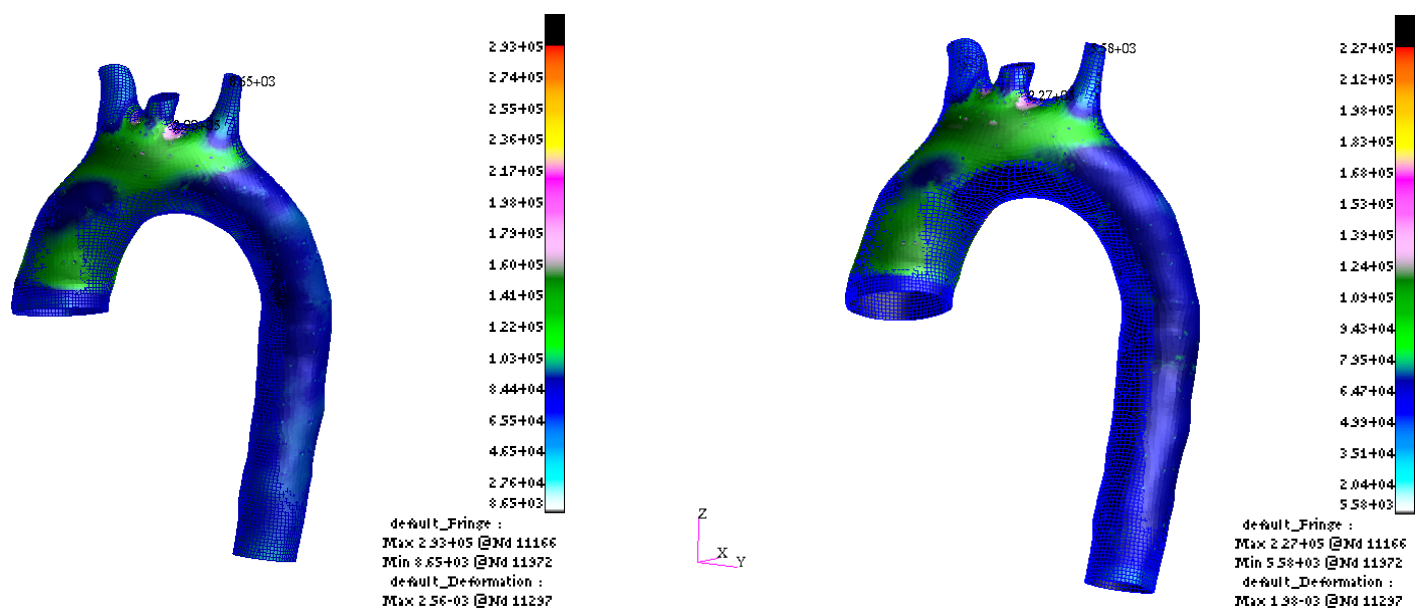

(a) Systolic von Mises stress under $1+\mathrm{Gz}$ load.

(b) Systolic von Mises stress under $6+\mathrm{Gz}$ load.

Figure 5.17: Comparison of systolic von Mises stress under inertial load.

\begin{tabular}{l|lll} 
CPU Cores & Wall-clock time & Average time per iteration & Data transfer per iteration \\
\hline 4 & $4 \mathrm{~h} 17 \mathrm{~m}$ & - & - \\
8 & $3 \mathrm{~h} 59 \mathrm{~m}$ & - & - \\
16 & $2 \mathrm{~h} 20 \mathrm{~m}$ & - & - \\
32 & $55 \mathrm{~m} 46 \mathrm{~s}$ & $5.21 \mathrm{~s}$ & $149.9 \mathrm{MB}$ \\
64 & $20 \mathrm{~m} 10 \mathrm{~s}$ & $2.51 \mathrm{~s}$ & $231.1 \mathrm{MB}$
\end{tabular}

Table 5.4: CFD solution scalability test conducted on the SHARCNET Saw supercomputer.

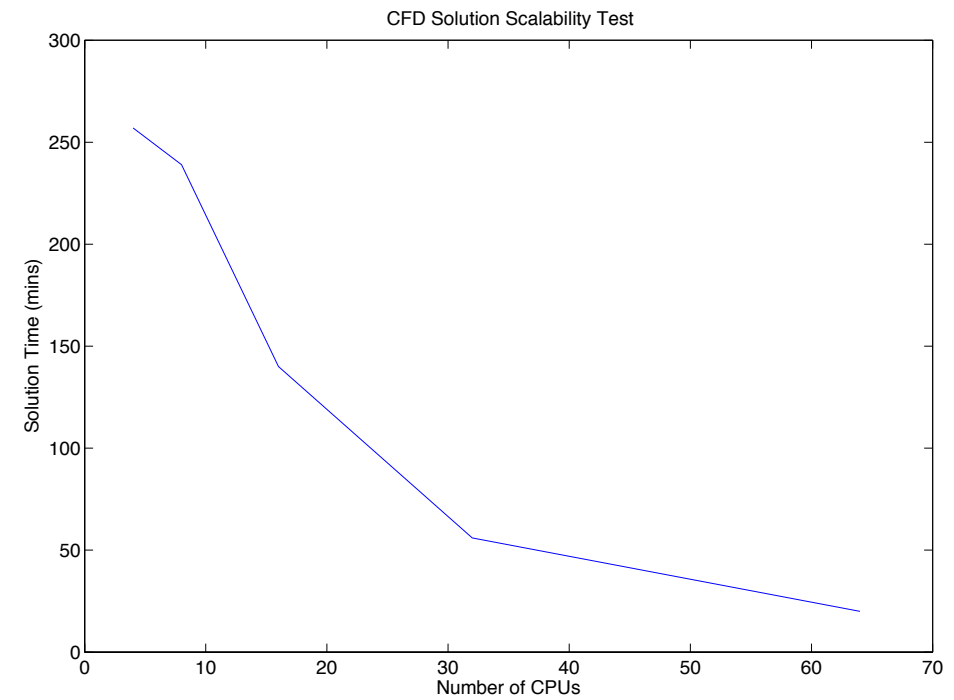

Figure 5.18: Scalability of the CFD solution in a parallel environment. 


\section{Chapter 6}

\section{Conclusions}

This thesis endeavoured to apply recent advances in medical imaging, mesh generation, and code-coupling methods to the field of aerospace physiology. It has been shown that a fully coupled fluid-structure interaction analysis using anatomically based geometry can be used to investigate the influence of inertial load on the aortic arch.

Some of the most complex steps involved with bio-mechanical simulations continue to include obtaining accurate geometries and suitable meshes. The use of idealized, CAD based geometry is convenient, although less useful when trying to capture flow nuances created by the organic nature of the structure. The accuracy of the results obtained in the presented research confirms that it is indeed feasible to utilize medical imaging to produce an anatomically correct, $\mathbb{N U R B S}$ based geometry suitable for use in a coupled analysis.

Along with obtaining suitable geometry, creating an effective mesh is equally challenging. Existing meshing algorithms are not well suited to organic curvature especially with regards to generating continuous inflation layers. The most effective solution to these challenges is to use an appropriate blocking scheme where the geometry is segmented into regions allowing separate mesh parameters to be used in regions with dramatic geometric change. If a patch style $\mathbb{D U K B S}$ reconstruction is used, one should also be aware of the boundaries between each patch and select appropriate meshing strategies accordingly. Such regions pose difficulties in meshing either the solid or fluid domains. With regards to the solid mesh specifically, the highly organic curvature of the geometry also created difficulties obtaining consistent element types (triangular or quadrilateral), which were necessary with the solver chosen in this analysis. The use of other solvers or configurations may mitigate this difficulty. With regards to the use of non-matching grids in the context of the fully coupled simulation, no appreciable difficulty was experienced.

Once an appropriate geometry has been obtained, it has been shown that unsteady CFD analysis can produce accurate results well within the range of those produced by experimental means. Acknowledg- 
ing this, it is believed that even higher fidelity can be achieved with the use of structured tree outflow boundary conditions, such as presented in Section 4. 2.2. Such an improvement would allow the investigation of the influence of $\mathrm{G}$ on pulsatile wave propagation throughout the great vessels. Although the $\mathrm{k}-\omega$ turbulence model chosen appeared to produce accurate results, an analysis into the use of other turbulence models such as large eddie models may be of academic interest.

Due to convergence issues, a linear elastic constitutive representation was chosen to represent the tissue behaviour of the aortic arch. This was realized with a noted discrepancy in wall displacement values as compared to experimental results. The results for tissue response presented should therefore be interpreted with this discrepancy in mind. The use of a constitutive model such as the nonlinear anisotropic model presented in Section 4.2 .3 should theoretically provide a more accurate representation of true tissue response.

With regards to the influence of inertial load, very limited information has been found for use in verification activities. The results should therefore be interpreted based on their accuracy in the standard gravity case with the following caveats. The results presented do not include the effects of physiologic compensation that naturally occur when the body is subject to a stress. These compensatory mechanisms include changes in heart rate, blood pressure, respiration rate, etc. Such mechanisms would act to oppose the applied load and maintain perfusion to vital organs such as the brain. These mechanisms however, often take several seconds to fully deploy, therefore these results should be interpreted as an instantaneous change in load, prior to any compensatory action. Second, this simulation does not model the entire cardiovascular system, which is a closed loop. Likewise, this model does not include any effects of inertial load on the venus return of blood to the heart. Further, it does not account for the volume shift and pooling that is known to occur as a result of the hydrostatic force. Additionally, the use of G-straining maneuvers or suits was not simulated.

\subsection{Improving Solution Efficiency}

The tightly coupled nature of the presented ESTl problem lends itself greatly to parallelization. All numerical regimes including the CFD, FEM, and code coupling operations have been designed to support parallel computing. The most effective use would be applied to the CFD solution as it is the most computationally expensive. Simple tests indicate that the mesh scales very well to at least $64 \mathrm{CPU}$ and likely beyond (Section 5.6). The use of clustered computing is strongly encouraged for future research.

One important remark regarding the deployment of such a parallel analysis as presented is the use of user defined boundary conditions. Although the FLUENT solver has been designed for domain segmentation and parallel efficiency, any user defined functions must also receive this attention and be written as efficient parallel routines using message passing interface (MPI) or Multi-Processing (OpenMP) as appropriate. 


\subsection{Future Analysis}

Although the results obtained in this investigation are preliminary, they illuminate an exciting range of applications for this field of research. One such avenue is the use of these results for determining an individual's tolerance of G. Conducting such tests numerically would have many benefits over traditional centrifuge based tests. Most human centrifuges are limited in the range of acceleration and the axis at which they are applied to the body. However, in practice, it is common for pilots and astronauts to be subjected to dynamic loading both in magnitude, direction, and time. The use of numerical techniques would allow $\mathrm{G}$ tolerance to be examined in any flight scenario. Future investigations could even integrate the $\mathrm{G}$ tolerance simulation into the vehicle flight dynamics model providing the most certainty regarding the safety of the crew throughout the flight profile.

Another fascinating application for the presented research is with regard to the influence of inertial load on the cardiovascular health of pilots and astronauts. The link between fluid parameters such as oscillatory wall shear stress and the development of cardiovascular disease has been postulated in many medical studies. In the presented results, the influence of inertial load on fluid behaviour such as secondary and helical flow formation has been demonstrated. It is then reasonable to suspect that a link may exist between applied inertial load and development or prevention of cardiovascular disease. It should be reiterated that substantial further analysis is required before these connections are verified. Nonetheless, the presented work provides exciting insight into this unexplored frontier of aerospace analysis. 



\section{Appendix A}

\section{Unsteady Velocity Relation}

This section defines the Fourier series used to describe the unsteady inlet velocity used in the presented analyses. This function was obtained by digitizing an experimental velocity plot obtained from Nichols et al. [57]. A Fourier curve fit analysis was then conducted in MATLAB (Mathworks Corp.) to obtain the presented coefficients.

$$
v(t)=a_{0}+a_{1} \cos (t \omega)+b_{1} \sin (t \omega)+\ldots+b_{8} \sin (8 t \omega) .
$$

Coefficients (with $95 \%$ confidence bounds):

$$
\begin{array}{ll}
a_{0}=2.094 & \\
a_{1}=-1.121 & b_{1}=0.3113 \\
a_{2}=-0.3412 & b_{2}=-0.2101 \\
a_{3}=-0.2507 & b_{3}=0.02114 \\
a_{4}=-0.1012 & b_{4}=0.00658 \\
a_{5}=-0.06782 & b_{5}=0.0001719 \\
a_{6}=-0.04863 & b_{6}=0.004727 \\
a_{7}=-0.03503 & b_{7}=0.005966 \\
a_{8}=-0.02637 & b_{8}=0.006643
\end{array}
$$

$\mathrm{w}=12.51$

R-square: 0.9929

Adjusted R-square: 0.9873 



\section{Appendix B}

\section{Additional Anatomic References}

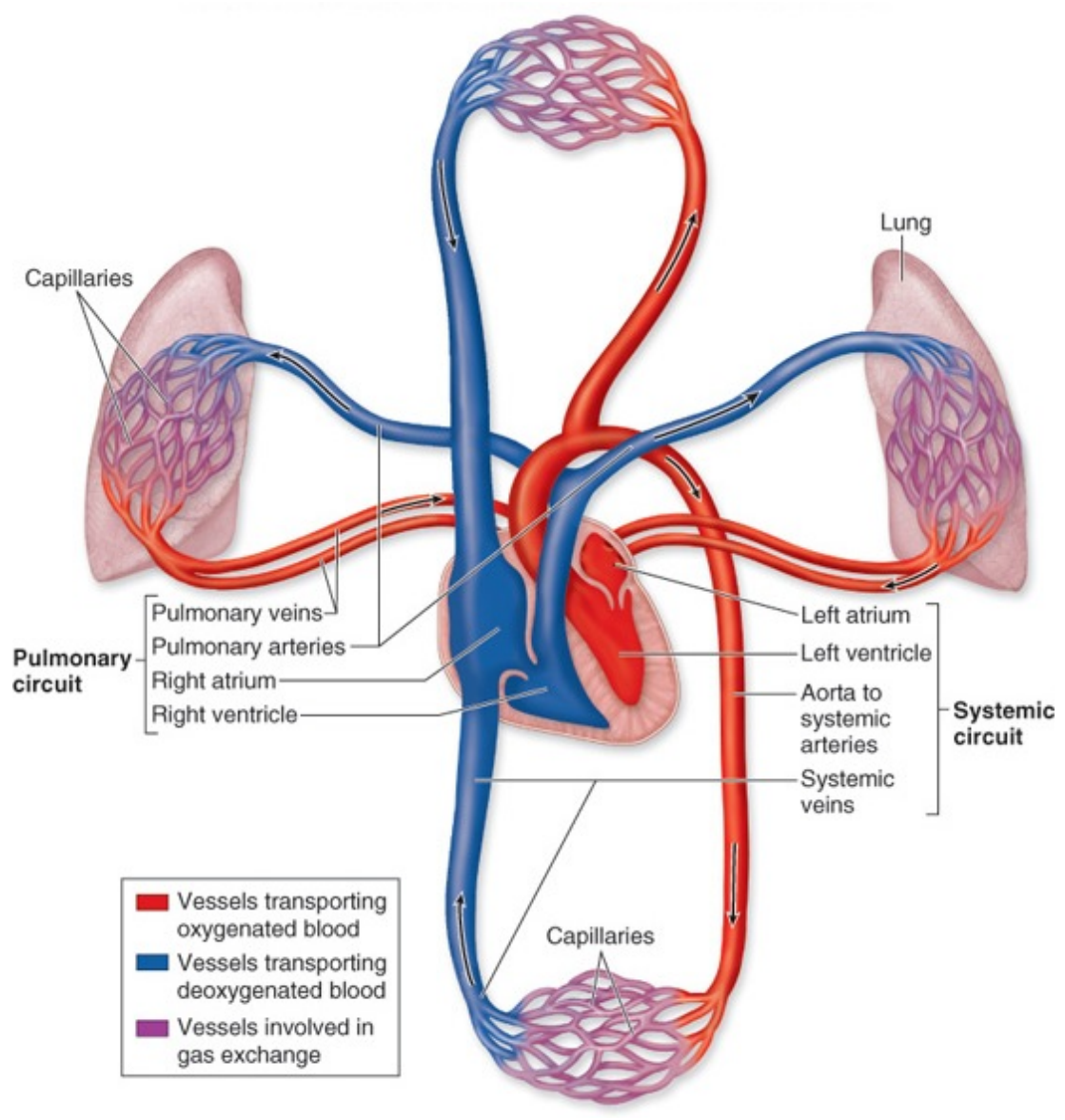

Figure B.1: Diagram of the cardiovascular system [5]. 


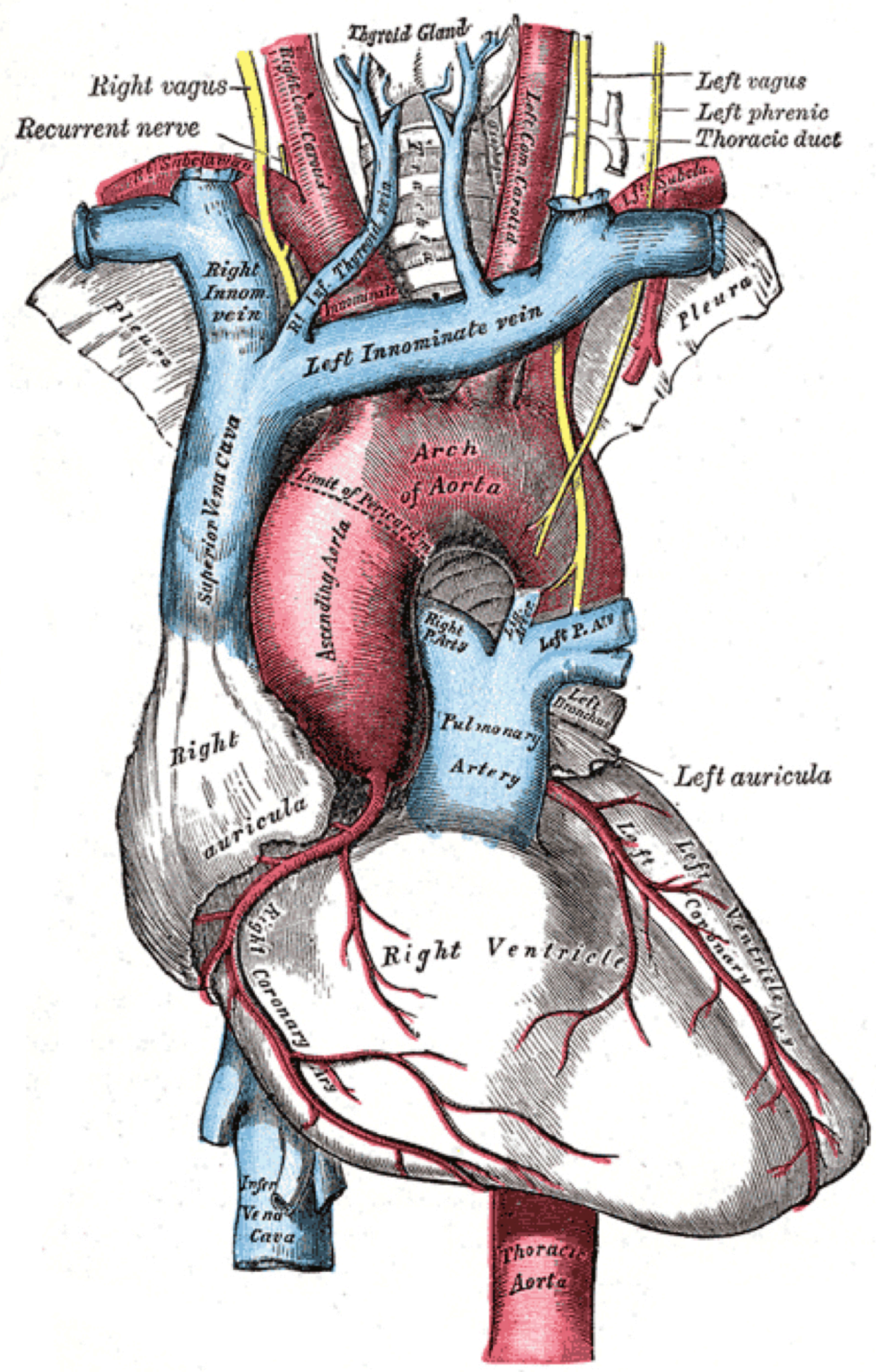

Figure B.2: Illustration of the heart and aortic arch [32]. 


\section{References}

[1] ANSYS CFX-Solver Theory. ANSYS Inc., Dec 2006.

[2] Theory reference for ANSYS and ANSYS Workbench. ANSYS Inc., 2007.

[3] Marc 2008 r1 User's Guide. MSC Software, 2008.

[4] Aeromedical Training for Flight Personnel, vol. FM 3-04.301(1-301). United States Department of the Army, 2009.

[5] The cardiovascular system [online]. 2009. Available from: http://academic.kellogg.cc.mi.us/ herbrandsonc/bio201_McKinley/f22-1_cardiovascular_sy_c.jpg [cited July 2010].

[6] MpCCI 4.0 Documentation. Fraunhofer SCAI, 2009.

[7] How MRI works [online]. July 2010. Available from: http://www.mrimagingblog.com/ how-mri-works/how-mri-works-2min-video [cited July 2010].

[8] ADAM. Components of blood [online]. 2009. Available from: http://www.pennmedicine.org/ health_info/bloodless/000209.html [cited July 2010].

[9] ADAM. Heart and valves [online]. 2009. Available from: http://m.medlineplus.gov/mlp/main/ rw/web/ency/imagepages/8672.htm [cited July 2010].

[10] AGARD. Short course on cardiopulmonary aspects of aerospace medicine. North Atlantic Treaty Organization. Advisory Group for Aerospace Reserach and Development, 1987.

[11] Barash, P. G. Clinical Anesthesia. Lippincott Williams And Wilkins, 2009.

[12] Bazilevs, Y., Gohean, J., Hughes, T., Moser, R., And Zhang, Y. Patient-specific isogeometric fluid-structure interaction analysis of thoracic aortic blood flow due to implantation of the Jarvik 2000 left ventricular assist device. Comput. Methods Appl. Mech. Engrg. (Jan 2009). Available from: http://linkinghub.elsevier.com/retrieve/pii/S0045782509001674.

[13] Beller, C., Labrosse, M., Thubrikar, M., and F.Robicsek. Finite element modeling of the thoracic aorta: including aortic root motion to evaluate the risk of aortic dissection. Journal of 
Medical Engineering 83 Technology (Jan 2008). Available from: http://www.informahealthcare. com/doi/abs/10.1080/03091900600687672.

[14] Beller, C. J., Labrosse, M. R., Thubrikar, M. J., Szabo, G., Robicsek, F., and Hagl, S. Increased aortic wall stress in aortic insufficiency: clinical data and computer model. European Journal of Cardio-Thoracic Surgery (Jan 2005). Available from: http://www.ejcts.ch/cgi/ content/abstract/27/2/270.

[15] Boresi, A. P., And Schmidt, R. J. Advanced Mechanics of Materials, 6th Ed. Jan 2009. Available from: http://books.google.com/books?id=hTHjQQAACAAJ\&printsec=frontcover.

[16] Bradley, W., Lisanti, C. J., And Hashemi, R. H. MRI: The Basics. Lippincott Williams And Wilkins, 2004.

[17] Bushong, S. C. Computed Tomography. McGraw-Hill, 2000.

[18] Carmody, C., Burriesci, G., Howard, I., and Patterson, E. An approach to the simulation of fluid-structure interaction in the aortic valve. Journal of Biomechanics (Jan 2006). Available from: http://linkinghub.elsevier.com/retrieve/pii/S0021929004005652.

[19] Chandran, K. B., Yoganathan, A. P., And Rittgers, S. E. Biofluid Mechanics: The Human Circulation. CRC Taylor \& Francis, 2007.

[20] Cheng, Y., Oertel, H., And Schenkel, T. Fluid-structure coupled CFD simulation of the left ventricular flow during filling phase. Ann Biomed Eng (Jan 2005). Available from: http: //www.springerlink.com/index/K1V52QMX17652W81.pdf.

[21] Daly, S., Prendergast, P., Dolan, F., and Lee, T. Use of finite element analysis to simulate the hyperelastic behavior of cardiovascular tissue. Proceedings of the 12th Conference of the European Society of Biomechanics (Jan 2000). Available from: http://ansys.net/ansys/papers/ cardiovascular.pdt.

[22] Davis, J. R., Johnson, R., And Stepanek., J. Fundamentals of Aerospace Medicine. Jan 2008. Available from: http://books.google.com/books?id=_6hymYAgC6MC\&printsec=frontcover.

[23] De Hart, J., Peters, G., Schreurs, P., and Banijens, F. A three-dimensional computational analysis of fluid-structure interaction in the aortic valve. Journal of Biomechanics (Jan 2003). Available from: http://linkinghub.elsevier.com/retrieve/pii/S0021929002002440.

[24] E Topol, R. C. Textbook of Cardiovascular Medicine. Lippincott Williams And Wilkins, 2007.

[25] F. Ghalichi, X. D. Low Reynolds number turbulence modelling of blood flow in arterial stenoses. Biorheology 35 (1998), 281-294.

[26] Frazin, L., Vonesh, M., and Chandran, K. Confirmation and initial documentation of thoracic and abdominal aortic helical flow: an ultrasound study. American Society of Artificial Inernal 
Organs (Jan 1996). Available from: http://journals.lww.com/asaiojournal/Abstract/1996/ 42060/Confirmation_and_Initial_Documentation_of_Thoracic.6.aspx.

[27] Fung, Y. Biomechanics: mechanical properties of living tissues. Jan 1993. Available from: http: //books. google.com/books? id=4HaMStTGOHwC\&printsec=frontcover.

[28] Fung, Y. Biomechanics: Circulation. Jan 1997. Available from: http://books.google.com/ books? Id=TlbXtdbT6D8C\&printsec= =trontcover.

[29] Gao, F., Guo, Z., Sakamoto, M., and Matsuzawa, T. Fluid-structure interaction within a layered aortic arch model. Journal of Biological Physics (Jan 2006). Available from: http: //wWw.springerlink.com/index/4X14U05860362M8U.pdf.

[30] Gao, F., And Watanabe, M. Stress analysis in a layered aortic arch model under pulsatile blood flow. BioMedical Engineering Online (Jan 2006). Available from: http://www.biomedcentral. com/content/pdf/1475-925X-5-25.pdf.

[31] Ghista, D., And Sandler, H. An analytic elastic-viscoelastic model for the shape and the forces in the left ventricle. Journal of Biomechanics (Jan 1969). Available from: http://linkinghub. elsevier.com/retrieve/pii/0021929069900402.

[32] Gray, H. The heart and aortic arch [online]. 1918. Available from: http://en.academic.ru/ pictures/enwiki/71/Gray505.png [cited July 2010].

[33] Green, J., And Miller, N. A model describing the response of the circulatory system to acceleration stress. Ann Biomed Eng (Jan 1973). Available from: http://www.springerlink.com/ index/J4277H736424830R.pdf.

[34] Holzapfel, G. Determination of material models for arterial walls from uniaxial extension tests and histological structure. Journal of Theoretical Biology (Jan 2006). Available from: http: //linkinghub.elsevier.com/retrieve/pii/S0022519305002080.

[35] Holzapfel, G., Eberlein, R., and Wriggers, P. Large strain analysis of soft biological membranes: Formulation and finite element analysis. Computer Methods in Applied Mechanics and Engineering (Jan 1996). Available from: http://linkinghub.elsevier.com/retrieve/pii/ 0045782596009991 .

[36] Holzapfel, G., And Gasser, T. A viscoelastic model for fiber-reinforced composites at finite strains: Continuum basis, computational aspects and applications. Computer Methods in Applied Mechanics and Engineering (Jan 2001). Available from: http://linkinghub.elsevier.com/ retrieve/pii/S0045782500003236.

[37] Holzapfel, G., Gasser, T., and Ogden, R. A new constitutive framework for arterial wall mechanics and a comparative study of material models. Journal of Elasticity (Jan 2000). Available from: http://www.springerlink.com/index/Q1185464175U2738.pdf. 
[38] Holzapfel, G., Sommer, G., Auer, M., Regitnig, P., And Ogden, R. W. Layer-specific 3D residual deformations of human aortas with non-atherosclerotic intimal thickening. Ann Biomed Eng (Jan 2007). Available from: http://www.springerlink.com/index/V8570V230774780U.pdf.

[39] Holzapfel, G. A. Computational biomechanics of soft biological tissue. Encyclopedia of Computational Mechanics (Jan 2004). Available from: http://www.biomech.tugraz.at/ files/publications/Holzapfel\%2520-\%2520Encyclopedia\%2520of\%2520Computational\% 2520Mechanics $\% 2520-\% 25202004 . p d f$.

[40] Hose, D., Narracott, A., Penrose, J., And Baguley, D. Fundamental mechanics of aortic heart valve closure. Journal of Biomechanics (Jan 2006). Available from: http://linkinghub. elsevier.com/retrieve/pii/S0021929005000655.

[41] Houghton, G. The Circulatory System. The Rosen Publishing Group, Inc, 2007.

[42] Jung, J., And Hassanein, A. Three-phase CFD analytical modeling of blood flow. Medical Engineering 65 Physics 30, 1 (Jan 2008), 91-103. Available from: http://linkinghub.elsevier. com/retrieve/pii/S1350453306002645.

[43] Kaufman, J. G. Properties of aluminum alloys: Fatigue data and the effects of temperature, product form, and processing. 559. Available from: http://books.google.com/books?id= Gt-CDab11KwC\&printsec=trontcover.

[44] Khanafer, K., Bull, J., And Berguer, R. Fluid-structure interaction of turbulent pulsatile flow within a flexible wall axisymmetric aortic aneurysm model. European Journal of Mechanics/B Fluids (Jan 2008). Available from: http://linkinghub.elsevier.com/retrieve/pii/ S(0.9.97(54608(0)(0)28.

[45] Kilner, P. J., Yang, G., Mohiaddin, R., Firmin, D., And Longmore, D. Helical and retrograde secondary flow patterns in the aortic arch studied by three-directional magnetic resonance velocity mapping. Circulation (Jan 1993). Available from: http://circ.ahajournals.org/cgi/ content/abstract/88/5/2235.

[46] Kim, H., Figueroa, C., Hughes, T., And Jansen, K. Augmented lagrangian method for constraining the shape of velocity profiles at outlet boundaries for three-dimensional finite element simulations of blood flow. Comput. Methods Appl. Mech. Engrg. (Jan 2009). Available from: http: //linkinghub.elsevier.com/retrieve/pii/S0045782509000887.

[47] Leuprecht, A., Kozerke, S., Boesiger, P., And K.Perktold. Blood flow in the human ascending aorta: a combined MRI and CFD study. Journal of Engineering Mathematics (Jan 2003). Available from: http://www.springerlink.com/index/KK640P7322806478.pdf.

[48] Lotz, J., Meier, C., Leppert, A., And Galanski, M. Cardiovascular flow measurement with phase-contrast MR imaging: Basic facts and implementation. Radiographics (Jan 2002). Available from: http://radiographics.rsnajnls.org/cgi/content/abstract/22/3/651. 
[49] Manoliu, V. Considerations about the lumped parameter windkessel model applicativity in the cardiovascular system structure. 1-9.

[50] Mirsky, I. Left ventricular stresses in the intact human heart. Biophysical Journal (Jan 1969). Available from: http://linkinghub.elsevier.com/retrieve/pii/S0006349569863794.

[51] Moore, J. A., Steinman, D. A., And Ethier, C. R. Computational blood flow modelling: Errors associated with reconstructing finite element models from magnetic resonance images. Journal of Biomechanics (Jan 2004). Available from: http://linkinghub.elsevier.com/retrieve/pii/ S0021929003003609.

[52] Morbiducci, U., Ponzini, R., Grigioni, M., And Redaelli, A. Helical flow as fluid dynamic signature for atherogenesis risk in aortocoronary bypass. a numeric study. Journal of Biomechanics (Jan 2007). Available from: http://linkinghub.elsevier.com/retrieve/pii/ S0021929006000741.

[53] Morbiducci, U., Ponzini, R., Rizzo, G., Cadioli, M., Esposito, A., Cobekki, F. D., MasChio, A. D., Montevecchi, F. M., And Redaello, A. In vivo quantification of helical blood flow in human aorta by time-resolved three-dimensional cine phase contrast magnetic resonance imaging. Ann Biomed Eng (Jan 2009). Available from: http://wWw.springerlink.com/index/ U7L66141L/44HVb8.pdf.

[54] Morris, L., Delassus, P., Callanan, A., And Walsh, M. 3D numerical simulation of blood flow through models of the human aorta. Journal of Biomechanical Engineering (Jan 2005). Available from: http://link.aip.org/link/?JBENDY/127/767/1.

[55] Mosora, F., Harmant, A., And Bernard, C. Modelling the arterial wall by finite elements. Archives of Physiology and Biochemistry (Jan 1993). Available from: http://www. informahealthcare.com/doi/abs/10.3109/13813459309046473.

[56] Naruse, T., And Tanishita, K. Large curvature effect on pulsatile entrance flow in a curved tube: model experiment simulating blood flow in an aortic arch. Journal of Biomechanical Engineering (Jan 1996). Available from: http://link.aip.org/link/?JBENDY/118/180/1.

[57] Nichols, W. W., O'Rourke, M. F., and McDonald, D. A. Mcdonald's blood flow in arteries: theoretic, experimental, and clinical principles. Hodder Arnold (Jan 2005), 607. Available from: http://books.google.com/books?id=F8RpQgAACAAJ\&printsec=frontcover.

[58] Okamoto, R., Wagenseil, J., Delong, W., and Peterson, S. Mechanical properties of dilated human ascending aorta. Ann Biomed Eng (Jan 2002). Available from: http://www. springerlink.com/index/P2GTR7616R423414.pdf.

[59] Olufsen, M. Structured tree outflow condition for blood flow in larger systemic arteries. American Journal of Physiology-Heart and Circulatory Physiology (Jan 1999). Available from: http://ajpheart.physiology.org/cgi/content/abstract/276/1/H257. 
[60] Olufsen, M., Peskin, C., Kim, W., Pedersen, E., Nadim, A., and Larsen, J. Numerical simulation and experimental validation of blood flow in arteries with structured-tree outflow conditions. Ann Biomed Eng (Jan 2000). Available from: http://www.springerlink.com/index/ UNT/518X1Q354/52.pdf.

[61] OsiriX. Dicom sample image set [online]. 2010. Available from: http://pubimage.hcuge.ch: 8080/ [cited July 11, 2010].

[62] Oyre, S., Pedersen, E., Ringgaard, S., Boesiger, P., and PaAske, W. In vivo wall shear stress measured by magnetic resonance velocity mapping in the normal human abdominal aorta. European Journal of Vascular Surgury (Jan 1997). Available from: http://linkinghub.elsevier. com/retrieve/pii/S1078588497800974.

[63] P I Aaronson, J. P. W. The cardiovascular system at a glance, vol. 2. Wiley-Blackwell, 2004.

[64] Pao, Y., Ritman, E., And Wood, E. Finite-element analysis of left ventricular myocardial stresses. Journal of Biomechanics (Jan 1974). Available from: http://linkinghub.elsevier. com/retrieve/pii/0021929074900815.

[65] Pedersen, E. M., Sung, H.-W., Burlson, A. C., And Yoganathan, A. P. Two-dimensional velocity measurements in a pulsatile flow model of the normal abdoinal aorta simulating different hemodynamic conditions. Journal of Biomechanics (Jun 2003), 11.

[66] Pittaccio, S., Migliavacca, F., Dubini, G., Morre-Pedersen, E., Frund, E.-T., HuortDAL, V., XU, Y., AND DELEval, M. Fluid-structure interaction and rigid wall CFD-MRI combined study of aortic coarctation repairs. 2003 Summer Bioengineering Conference, June 25-29 (Jan 2003). Available from: http://www.tulane.edu/ sbc2003/pdfdocs/0509.PDF.

[67] Prosi, M., Perktold, K., Ding, Z., and Friedman, M. Influence of curvature dynamics on pulsatile coronary artery flow in a realistic bifurcation model. Journal of Biomechanics (Jan 2004). Available from: http://linkinghub.elsevier.com/retrieve/pii/S0021929004000533.

[68] Ranga, A., Mongrain, R., Galaz, R., Biadillah, Y., and Cartier, R. Large-displacement 3D structural analysis of an aortic valve model with nonlinear material properties. Journal of Medical Engineering \& Technology (Jan 2004). Available from: http://www.informaworld.com/ index/713825403.pdf.

[69] Rideout, V., And Katra, J. Computer simulation study of the pulmonary circulation. Simulation (Jan 1969). Available from: http://sim.sagepub.com/cgi/reprint/12/5/239.pdf.

[70] Ryval, J., Straftman, A., and Steinman, D. Two-equation turbulence modeling of pulsatile flow in a stenosed tube. Journal of Biomechanical Engineering (Jan 2004). Available from: http: //link.aip.org/link/?JBENDY/126/625/1. 
[71] Sabbah, H., And Stein, P. Turbulent blood flow in humans: its primary role in the production of ejection murmurs. Circulation Research (Jan 1976). Available from: http://circres. ahajournals.org/cgi/content/abstract/38/6/513.

[72] Schetz, J. A. Boundary layer analysis. 586. Available from: http://books.google.com/books? id=vcNvQgAACAAJ\&printsec $=$ Irontcover.

[73] Segers, P., Rietzschel, E., And Buyzere, M. D. Three and four-element windkessel models: assessment of their fitting performance in a large cohort of healthy middle-aged individuals. Proceedings of the Institution of Mechanical Engineers (Jan 2008). Available from: http://journals.pepublishing.com/index/TG14W49585Q03241.pdf.

[74] Shadban, A., And Duerinckx, A. Wall shear stress and early atherosclerosis: a review. American Journal of Radiology (Jan 2000). Available from: http://www.ajronline.org/cgi/content/ full/174/6/1657.

[75] Shahcheraghi, N., Dwyer, H., Cheer, A., and Barakat, A. Unsteady and three-dimensional simulation of blood flow in the human aortic arch. Journal of Biomechanical Engineering (Jan 2002). Available from: http://link.aip.org/link/?JBENDY/124/378/1.

[76] Shim, Y., Pasipoularides, A., and Straley, C. Arterial windkessel parameter estimation: a new time-domain method. Ann Biomed Eng (Jan 1994). Available from: http: //wWw.springerlink.com/index/PR3023RWVW417723.pdf.

[77] Snyder, M., Rideout, V., and Hillestad, R. Computer modeling of the human systemic arterial tree. Journal of Biomechanics (Jan 1968). Available from: http://linkinghub.elsevier. com/retrieve/pii/0021929068900298.

[78] Stergiopulos, N., Westerhof, B., and Westerhof, N. Total arterial inertance as the fourth element of the windkessel model. American Journal of Heart and Circulation Physiology (Jan 1999). Available from: http://ajpheart.physiology.org/cgi/content/abstract/276/1/H81.

[79] Svensson, J., Gardhagen, R., Heiberg, E., Ebbers, T., Loyd, D., Lanne, T., and Karlsson, M. Feasibility of patient specific aortic blood flow CFD simulation. MICCAI 2006 (Jan 2006). Available from: http://www.springerlink.com/index/440114lu823176n4.pdf.

[80] Taylor, C., Hughes, T., And Zarins, C. Finite element modeling of three-dimensional pulsatile flow in the abdominal aorta: Relevance to atherosclerosis. Ann Biomed Eng (Jan 1998). Available from: http://www.springerlink.com/index/Q4R26W6443676M75.pdf.

[81] Tezduyar, T., Sathe, S., Schwaab, M., And Conklin, B. Arterial fluid mechanics modeling with the stabilized space-time fluid-structure interaction technique. International Journal for $\mathrm{Nu}$ merical Methods in Fluids (Jan 2007). Available from: http://www3.interscience.wiley.com/ journal/116332039/abstract. 
[82] Tripp, L. Cerebral blood flow during $+\mathrm{Gz}$ acceleration as measured by transcranial doppler. The Journal of Clinical Pharmacology (Jan 1991). Available from: http://jcp.sagepub.com/cgi/ reprint/31/10/911.pdf.

[83] van Loon, R., Anderson, P., And van de Vosse, F. A fluid-structure interaction method with solid-rigid contact for heart valve dynamics. Journal of Computational Physics (Jan 2006). Available from: http://linkinghub.elsevier.com/retrieve/pii/S0021999106000416.

[84] Varghese, S., And Frankel, S. Numerical modeling of pulsatile turbulent flow in stenotic vessels. Journal of Biomechanical Engineering (Jan 2003). Available from: http://link.aip.org/link/ ? JBENDY/125/445/1.

[85] Venkatasubramaniam, A., Fagan, M., Mehta, T., Mylankal, K. J., Ray, B., Kuhan, G., Chetter, I. C., And McCollum, P. T. A comparative study of aortic wall stress using finite element analysis for ruptured and non-ruptured abdominal aortic aneurysms. European Journal of Vascular Surgury (Jan 2004). Available from: http://linkinghub.elsevier.com/retrieve/pii/ 51078588404001789 .

[86] Vignon, I., and TAYlor, C. Outflow boundary conditions for one-dimensional finite element modeling of blood flow and pressure waves in arteries. Wave Motion (Jan 2004). Available from: http://linkinghub.elsevier.com/retrieve/pii/S0165212503001173.

[87] Waite, L. Biofluid Mechanics in Cardiovascular Systems. McGraw-Hill, 2006.

[88] Wang, J., Flewitt, J., Shrive, N., Parker, K., and Tyberg, J. V. Waves propagating on a windkessel: relation of arterial and venous windkessels to systemic vascular resistance. American Journal of Physiology- Heart and Circulatory Physiology (Jan 2006). Available from: http:// ajpheart.physiology.org/cgi/content/abstract/290/1/H154.

[89] Wang, J.-J., O’brien, A., Shrive, N., Parker, K., and Tyberg, J. Time-domain representation of ventricular-arterial coupling as a windkessel and wave system. AJP - Heart and Circulatory Physiology 284, 4 (Apr 2003), H1358. Available from: http://ajpheart.physiology.org/cgi/ content/full/284/4/H1358.

[90] Wentzel, J., Corti, R., Fayad, Z., and Wisdom, P. Does shear stress modulate both plaque progression and regression in the thoracic aorta? Human study using serial magnetic resonance imaging. Journal of the American College of Cardiology (Jan 2005). Available from: http:// linkinghub.elsevier.com/retrieve/pii/S0735109704025112.

[91] Westerhof, N., Lankhaar, J., and Westerhof, B. The arterial windkessel. Medical and Biological Engineering and Computing (Jan 2009). Available from: http://wWw.springerlink. com/index/78L470N5J0V28U77.pdf.

[92] Wolters, B., Rutten, M., Schurink, G., Kose, U., Hart, J. D., and van de Vosse, F. A patient-specific computational model of fluid-structure interaction in abdominal aortic aneurysms. 
Medical Engineering and Physics (Jan 2005). Available from: http://linkinghub.elsevier.com/ retrieve/pii/S1350453305001396.

[93] Wood, E. H., Sutterer, W. F., Marshall, S. H. W., Lindberg, E. F., and Headley, R. N. Effect of headward and forward accelerations on the cardiovascular system. WADD Technical Report 60-634 (1961). Available from: http://handle.dtic.mil/100.2/AD255298.

[94] ZAmiR, M. The physics of pulsatile flow. 2000. Available from: http://books.google.com/books? id=8ZdtgTjrWIQC\&printsec=trontcover. 



\section{Glossary}

arterioles Very small arterial branches.

atherogenesis The development of plaque in arteries. 5

automaticity Spontaneous discharge of cardiac cells causing the heart to contract. B

capillaries Microscopic blood vessels where cellular gas exchange occurs.

compliance Elastic deformability of an artery wall. 27

hematocrit Ratio of the volume of red blood cells to total volume of blood. ㄸ

hemodynamics The study of the fluid dynamics of blood.

hypoxia Reduced perfusion of oxygen to body organs and tissues. [, [ $]$

perfusion The transfer of oxygen and nutrients to body organs and tissues. $\square, 0.5$

peripheral vascular resistance Flow resistance created by the progressive narrowing of downstream vasculature. [

prolapse The collapse of valve leaflets into the preceding chamber. 团

regurgitation Leaking of a cardiac valve allowing back flow of blood. [4, [26]

stroke volume Volume of blood pumped by the left ventricle. $\square$

strong coupling Governing equations in a multi-physics problem are solved in a integrated fashion. 34 systole The ejection phase of the cardiac cycle. [3

systolic pressure Blood pressure during the ejection (pulse) phase of the cardiac cycle. 38 
vasculature The network of blood vessels throughout the body. [

weak coupling Governing equations in a multi-physics problem are solved in a segregated fashion. 34 


\title{
Acronyms
}

\author{
ALE arbitrary Lagrangian-Eularian. [13 \\ CAD computer aided design. [प], [.9] \\ CFD computational fluid dynamics. [2, [2, [7, [1] \\ CT computed tomography. ॠ, Ш, ए2, ए7 एप \\ FD fictitious domain. 피

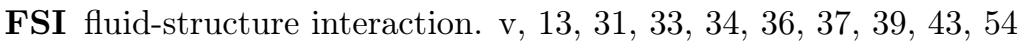

MpCCI Mesh-based Parallel Code Coupling Interface. B4, [3]

MPI message passing interface. 54

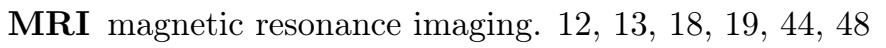

NURBS non-uniform rational basis splines. 21, [22, [5] 



\section{Index}

action potential, $\mathbf{3}$

aerospace medicine, $\mathrm{\square}$

aortic valve, [1, [2.5.

artery, 回

atria, [3

atrioventricular valves, 田

automaticity, [3

blackout, [6]

boundary layer, 2.3

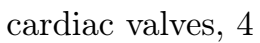

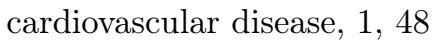

cardiovascular system, 田, [3, [3]

centrifuge, 四

compensatory mechanisms, [

convergence, 39

CT (Computed tomography), 미

elasticity, 28

flow rate, 27

FLUENT, [2], 34, 54

Fourier series, 20.

G suit, [

$\mathrm{G}$ tolerance, 四

heart, 3

heart rate, [26]

helical flow, 48 helicity, [2.3]

hemodynamics,

hydrostatic equation, $\mathbf{6}$

hyperelasticity, $30-33$

hypoxia, [6, [ $]$

image segmentation, 2$]$

impedance, [2]

inertial load, 四

interpolation, 34

jvlNet, 留

k- $\omega$ model, 30

Kd-tree, 36

lumped parameter models, 27

mass flow rate, 미

medical imaging, ㅁ.

MpCCI, [2, 34, 36]

MSC. Marc, ఇ, उय

Navier-Stokes equations, 28

non-conforming grids, 36

non-newtonian, 田, 8

NURBS, [T]

perfusion, [

peripheral vascular resistance, $\mathbf{\square}$

phase lag, 1,28 
physiology, س

plasma, 国

platelets, 回

poiseuille flow, $\square$

prolapse, 四

pulsatile flow, 8

recirculating flow, 48

resistance, 27

Reynolds number, $\mathbf{\nabla}, \mathbf{]}, \mathbf{1}, \mathbf{2 : 3}, \mathbf{3}]$

secondary flow, 48

semilunar valve, 四

SHARCNET, 5

shell elements, $B$ [
Steady Solution, $\mathbf{3 7}$

strong coupling, 34

systole, [3, 48

turbulence modelling, [】]

vasculature, 1

velocity profile, 48

ventricle, 3,8

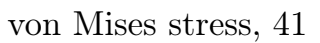

wall shear stress, m

wave motion, $\mathbf{9}$

wave propagation, $[28$

weak coupling, 34

windkessel, 28 